\title{
Classifying automorphic representations
}

\author{
James Arthur
}

\begin{abstract}
We shall describe a recent classification of the automorphic representations of orthogonal and symplectic groups. In order to place it in perspective, we shall also review Langlands' general principle of functoriality and related matters. Finally, we shall add a few remarks on the explicit application of the results to those cases of functoriality on which they bear.
\end{abstract}

\section{Contents}

Preface

1. Classical groups and functoriality

2. The automorphic Langlands group $L_{F}$

4. The case of $G L(N) \quad 21$

5. Global parameters for $G \quad 30$

6. Transfer and the fundamental lemma 38

7. Statement of theorems 44

8. Implications for functoriality $\quad 51$

$\begin{array}{ll}\text { References } & 56\end{array}$

\section{Preface}

This article is an introduction to the monograph [ECR], the purpose of which was to classify the automorphic representations of a family of classical groups. The groups are quasisplit, special orthogonal and symplectic groups $G$. Their representations are classified in terms of those of general linear groups $G L(N)$. The monograph is based on the stabilization of the trace formula for $G$, established for any connected group in [A1]. It also depends on the stabilization of the twisted trace formula for $G L(N)$, which represents work in progress by Moeglin and Waldspurger [W5]-[W7], [MW2]. Until it has been completed, the classification will remain conditional.

There are already two short surveys [A4], [A5] of some of the main results of $[\mathbf{E C R}]$. This article is somewhat different. I have tried to write it as a longer

2010 Mathematics Subject Classification. Primary 11F70, 11F66. 
report that might be suitable for the broader readership of Current Developments in Mathematics. The monograph $[\mathbf{E C R}]$ is long, and often quite complex. It draws on techniques from many diverse sides of the subject, which would be hard to present in any detail here. Moreover, what has been included in this report might still be difficult in places. I hope, however, that I have given enough motivation to offer some perspective on the modern theory of automorphic forms, as well as the actual contents of $[\mathbf{E C R}]$.

For the most part, we confine ourselves to the orthogonal and symplectic groups $G$ whose representations we classify. We recall these groups in $\S 1$, and their relations with Langlands' principle of functoriality. In $\S 2$, we discuss the automorphic Langlands group $L_{F}$. Its existence is far from known, but its expected properties offer much guidance. In $\S 3$, we describe how Langlands parameters and their generalizations, which in their global form would be defined on the hypothetical group $L_{F}$, suggest how to relate representations of $G$ with those of general linear groups $G L(N)$. This simple exercise in linear algebra also provides an entry into the theory of endoscopy, which underlies the statements (and proofs) of our theorems.

The representation theory of $G L(N)$ is relatively simple, and quite well understood now, thanks to the work of a number of mathematicians over the past forty years. We shall review some of it in $\S 4$, taking the opportunity also to review the theory of arithmetic and automorphic $L$-functions. We shall use the automorphic representations of $G L(N)$ in $\S 5$ as a foundation for ad hoc global parameters for $G$ that do not depend on the hypothetical group $L_{F}$. The construction requires two "seed" theorems (Theorems 5.1 and 5.2), which we state but (like everything else) do not prove.

In $\S 6$, we review the theory of endoscopy, whose spectral roots we encountered in $\S 3$. We use it to state a critical local result (Theorem 6.1), which is the starting point for the local classification. We will then be in a position to state the main theorems (Theorems 7.1, 7.2 and 7.3) in $\S 7$. In the final $\S 8$, we will add some supplementary comments on how the theorems relate to the two fundamental cases of functoriality discussed in $\S 1$. These observations do not appear in [ECR].

We follow the discussion from [ECR] closely in some places, and reorder it in others. We are also including supplementary background material from the theory of automorphic forms and the Langlands program. We have not tried to crossreference statements here with those of $[\mathbf{E C R}]$, but a reader will have no difficulty seeing how they correspond. This report takes us through Sections 1.1-2.1 (and the beginning of $\S 2.2$ ) of [ECR], the part of the monograph given to the statements of the main theorems. Most of the rest of the monograph, specifically Sections 2.3-8.2 (and the remaining part of $\S 2.2$ ), is devoted to the proofs. The argument is long and complex, but it has a certain unity. It comes with several layers of induction, of which we will see hints in $\S 5$. We will add nothing further to this, except to note that the heart of the argument is an endoscopic comparison of trace formulas.

Acknowledgments. The author was supported in part by NSERC Grant A3483.

\section{Classical groups and functoriality}

The groups we consider will be attached to the four infinite families of complex simple Lie algebras. These are represented by the following four infinite families of Coxeter-Dynkin diagrams, for which I am indebted to W. Casselman. 


\section{Type $A_{n}$}

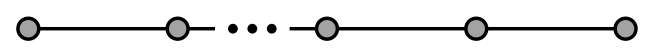

$\underline{\text { Type } B_{n}}$

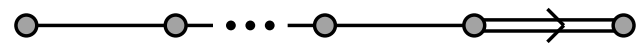

$\underline{\text { Type } C_{n}}$

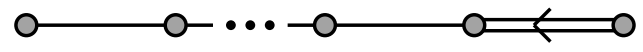

\section{Type $D_{n}$}

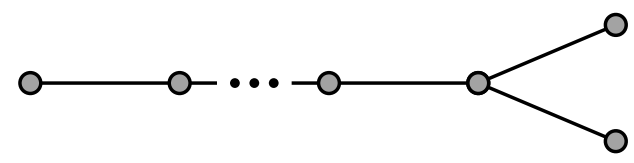

For corresponding complex groups, we could take the special linear groups $S L(n+$ $1, \mathbb{C})$, the odd orthogonal groups $S O(2 n+1, \mathbb{C})$, the symplectic groups $S p(2 n, \mathbb{C})$ and the even orthogonal groups $S O(2 n, \mathbb{C})$. The family $\mathbf{A}_{n}$ will be our starting point. Since the theory here is simplest for general linear groups, we will take the reductive groups $G L(N, \mathbb{C}), N=n+1$, as the complex representatives for this family.

We actually want to take these groups over a number field $F$ (such as the rational numbers $\mathbb{Q}$ ) or one of its completions (such as the real number field $\mathbb{R}$ or a $p$-adic field $\mathbb{Q}_{p}$ ). We therefore take $F$ to be any local or global field of characteristic 0 .

We recall the fundamental theorem of Chevalley, which implies that any of these complex groups has a canonical $F$-structure. In other words, for each of the diagrams, there is a canonical classical group that is defined over $F$. It is the split group attached to the given diagram (and centre). Our interest is actually in quasisplit groups. They represent a broader class of groups, obtained by twisting any given split group by a Galois action on the diagram. The symmetry group of a diagram is the group of bijections of the set of vertices that preserve all of the edges and arrows. It is isomorphic to $\mathbb{Z} / 2 \mathbb{Z}$ in type $\mathbf{A}_{n}$, trivial in types $\mathbf{B}_{n}$ and $\mathbf{C}_{n}$, and equal ${ }^{1}$ to $\mathbb{Z} / 2 \mathbb{Z}$ in type $\mathbf{D}_{n}$. A quasisplit group is determined by a homomorphism from the Galois group

$$
\Gamma_{F}=\Gamma_{\bar{F} / F}=\operatorname{Gal}(\bar{F} / F)
$$

\footnotetext{
${ }^{1}$ If $n=4$, this group is actually isomorphic to $S_{3}$, but we agree to consider only the standard symmetries that interchange the two right hand vertices in the diagram.
} 
of an algebraic closure $\bar{F}$ over $F$ to the symmetry group of the diagram. The monograph $[\mathbf{E C R}]$ does not treat nonsplit, quasisplit groups of type $\mathbf{A}_{n}$. (These are unitary groups, for which we refer the reader to [Mok].) Since a quasisplit group of type $\mathbf{B}_{n}$ or $\mathbf{C}_{n}$ is split, we have only to consider type $\mathbf{D}_{n}$. In this case, a quasisplit group is determined by a quotient of $\Gamma_{F}$ of order 1 or 2 , or in other words, a Galois extension $E / F$ of degree 1 or 2 .

One of the remarkable discoveries of Langlands has been the fundamental role played by a certain dual group. We take $G$ to be the group over $F$ we are working with, either a general linear group $G L(N)$ or a quasisplit special orthogonal or symplectic group. The dual group $\widehat{G}$ is a complex classical group, which is attached to the dual diagram obtained by reversing the directions of any arrows in the diagram of $G$. Chevalley's theorem is based on an identification of the symmetry group of the diagram with the group of outer automorphisms of the split group (or rather, a group of $F$-automorphisms that represent those outer automorphisms). This transfers to a dual Galois action of $\Gamma_{F}$ by complex analytic automorphisms of $\widehat{G}$, which factors through the quotient

$$
\Gamma_{E / F}=\operatorname{Gal}(E / F)
$$

of $\Gamma_{F}$. Langlands built this action into the dual group by forming the semidirect product

$$
{ }^{L} G=\widehat{G} \rtimes \Gamma_{F}
$$

that is now known as the $L$-group.

The $L$-group is actually a more concrete object than might be suggested by the large Galois factor. For many purposes, one can replace $\Gamma_{F}$ by any quotient $\Gamma_{E / F}$ through which the Galois action factors, and in particular, by the minimal such quotient above. If $G$ is split, for example, one can often take $E=F$ and ${ }^{L} G=\widehat{G}$. Equipped with this minimal form ${ }^{L} G={ }^{L} G_{E / F}$ of the $L$-group, our four families of groups are then as follows.

Type $\mathbf{A}_{\mathbf{n}}: G=G L(N)$ is split, and $\widehat{G}=G L(N, \mathbb{C})={ }^{L} G$, where $N=n+1$.

Type $\mathbf{B}_{\mathbf{n}}: G=S O(2 n+1)$ is split, and $\widehat{G}=S p(2 n, \mathbb{C})={ }^{L} G$.

Type $\mathbf{C}_{\mathbf{n}}: G=S p(2 n)$ is split, and $\widehat{G}=S O(2 n+1, \mathbb{C})={ }^{L} G$.

Type $\mathbf{D}_{\mathbf{n}}: G=S O(2 n)$ is quasisplit, and $\widehat{G}=S O(2 n, \mathbb{C})$;

$$
{ }^{L} G=S O(2 n, \mathbb{C}) \rtimes \Gamma_{E / F}, \text { where } \operatorname{deg}(E / F) \in\{1,2\} .
$$

To keep the scope of the article within bounds, we will generally confine our discussion to groups from this list. Unless otherwise stated, groups $G, G^{\prime}$, etc. will be assumed to be taken from our four families of quasisplit classical groups, or possibly to be direct products of such groups. In fact, we will often restrict $G$ to be of type $\mathbf{B}_{n}, \mathbf{C}_{n}$ or $\mathbf{D}_{n}$, and explicitly write $G L(N)$ for any one of our groups of type $\mathbf{A}_{n}$.

We are interested in the representation theory of $G$. If $F$ is local, our concern will be the set $\Pi(G)$ of equivalence classes of irreducible representations of $G(F)$, together with its subsets

$$
\Pi_{\text {temp }}(G) \subset \Pi_{\text {unit }}(G) \subset \Pi(G)
$$


of representations that are respectively tempered and unitary. (A tempered representation can be described informally as an irreducible representation $\pi$ such that the tensor product

$$
\pi \otimes \pi^{\vee}, \quad \pi^{\vee}(x)={ }^{t} \pi(x)^{-1}, x \in G(F),
$$

occurs in the decomposition of the regular representation

$$
\left(R\left(y_{1}, y_{2}\right) \phi\right)(x)=\phi\left(y_{1}^{-1} x y_{2}\right), \quad x, y_{1}, y_{2} \in G(F), \phi \in L^{2}(G(F)),
$$

of $G(F) \times G(F)$ on the Hilbert space $L^{2}(G(F))$. It is automatically unitary.) If $F$ is global, we are interested in the set $\Pi(G)$ of automorphic representations of $G$. These are irreducible representations of the adelic group $G(\mathbb{A})$, which are of a very special sort.

We recall that the adeles $\mathbb{A}=\mathbb{A}_{F}$ of $F$ form a locally compact ring, in which $F$ embeds as a discrete subring. The adelic group is then a restricted direct product

$$
G(\mathbb{A})=\prod_{v}^{\sim} G\left(F_{v}\right),
$$

taken over the valuations $v$ on $F$. For any $v, F_{v}$ is the locally compact field obtained by completing $F$ with respect to $v$. It is modeled on the standard case of the completion $F_{v}=\mathbb{R}$ of $F=\mathbb{Q}$ with respect to the usual absolute value $|\cdot|_{v}=|\cdot|$. We recall that the complementary valuations for $F=\mathbb{Q}$ are the nonnegative functions

$$
|u|_{p}= \begin{cases}p^{-r}, & \text { if } u=\frac{a}{b} p^{r}, \text { for } a, b, r \in \mathbb{Z}, \quad(a, p)=(b, p)=1, \\ 0, & \text { if } u=0,\end{cases}
$$

on $\mathbb{Q}$, parametrized by prime numbers $p$. In general, the restricted direct product is the group of elements

$$
x=\prod_{v} x_{v}, \quad x_{v} \in G\left(F_{v}\right)
$$

in the direct product such that for almost all valuations $v, x_{v}$ lies in the maximal compact subgroup $G\left(\mathfrak{o}_{v}\right)$ of points in $G\left(F_{v}\right)$ with values in the compact subring

$$
\mathfrak{o}_{v}=\left\{u_{v} \in F_{v}:\left|u_{v}\right|_{v} \leq 1\right\}
$$

of integers in $F_{v}$. It becomes a locally compact group under the appropriate direct limit topology. The group $G(F)$ embeds in $G\left(F_{v}\right)$ (as a dense subgroup). The diagonal embedding of $G(F)$ into $G(\mathbb{A})$ exists (because an element in $G(F)$ is integral at almost all valuations $v)$, and is easily seen to have discrete image.

Since $G(F)$ is discrete in $G(\mathbb{A})$, the quotient $G(F) \backslash G(\mathbb{A})$ is a reasonable object. It comes with a right invariant measure, which is determined up to a positive multiplicative constant. One can therefore form the associated space $L^{2}(G(F) \backslash G(\mathbb{A}))$ of square-integrable functions. It is a Hilbert space, equipped with the unitary representation

$$
(R(y) \phi)(x)=\phi(x y), \quad x, y \in G(\mathbb{A}), \phi \in L^{2}(G(F) \backslash G(\mathbb{A})),
$$

of $G(\mathbb{A})$ by right translation. An automorphic representation is an irreducible representation of $G(\mathbb{A})$ that occurs in the spectral decomposition of $R$.

The description of an automorphic representation just given is more of an informal characterization than a definition. It can be made precise, but it is also 
more restrictive than the formal definition ${ }^{2}$ in $[\mathbf{L 3}]$. The representation $R$ has a subrepresentation $R_{\text {disc }}$ that decomposes discretely ${ }^{3}$ into a direct sum of irreducible representations (like the 1-dimensional representations

$$
x \longrightarrow e^{2 \pi i n x}, \quad x \in \mathbb{R}, n \in \mathbb{Z},
$$

from the theory of Fourier series), and a complementary subrepresentation $R_{\text {cont }}$ that decomposes continuously into a direct integral of irreducible representations (like the 1-dimensional representations

$$
x \longrightarrow e^{\lambda x}, \quad x \in \mathbb{R}, \lambda \in i \mathbb{R},
$$

from the theory of Fourier transforms). The point is that an automorphic representation can occur in either the discrete or the continuous spectrum. In another sense, however, the analogy with classical Fourier analysis is misleading. This is because an automorphic representation $\pi$ of $G(\mathbb{A})$ has much more structure than these familiar 1-dimensional representations. According to $[\mathbf{F}]$, any such $\pi$ can be written as a restricted tensor product

$$
\pi=\widetilde{\bigotimes}_{v} \pi_{v}, \quad \pi_{v} \in \Pi_{\text {unit }}\left(G_{v}\right)
$$

of irreducible unitary representations $\pi_{v}$ for the local groups $G_{v}$ over $F_{v}$. It is one thing to be able to put together an irreducible representation $\pi$ of $G(\mathbb{A})$ explicitly as a product of this sort. We would require some knowledge of the sets $\Pi_{\text {unit }}\left(G_{v}\right)$, an interesting problem to be sure, and one that has not been solved in general, but nothing further. It is quite another matter to determine which such products are automorphic. This new constraint imposes profound relations among the different constituents $\pi_{v}$ of $\pi$.

In fact, it is pretty clear that we will never be able to write down all automorphic representations explicitly. Langlands' point of view was more subtle. He discovered unexpected reciprocity laws among the automorphic representations for different groups. Thus, although an explicit construction of all automorphic representations will not be available, there will still be hidden ties that bind different automorphic representations. Langlands formulated them as a fundamental principle $[\mathbf{L} \mathbf{1}]$, which later became known as functoriality. It pertains to any analytic homomorphism $\rho$ between two $L$-groups ${ }^{L} G^{\prime}$ and ${ }^{L} G$, taken as groups over the full Galois group $\Gamma_{F}$, that commutes with the two projections onto $\Gamma_{F}$. (A homomorphism with these properties is called an L-homomorphism.)

PRINCIPLE 1.1 (Langlands Functoriality). Suppose that $G$ and $G^{\prime}$ are general quasisplit groups over a number field $F$. Then any L-homomorphism

$$
\rho:{ }^{L} G^{\prime} \longrightarrow{ }^{L} G
$$

between their L-groups determines a natural correspondence

$$
\pi^{\prime} \longrightarrow \pi
$$

\footnotetext{
${ }^{2}$ This refers to the definition on p. 203 of [L3] that was shown in Proposition 2 of $[\mathbf{L 3}]$ to be equivalent to the formal definition of an automorphic representation in $[\mathbf{B J}]$.

3 To be correct, we should really be speaking of the relative discrete spectrum. That is, $R_{\text {disc }}$ is the representation of $G(\mathbb{A})$ on the invariant subspace $L_{\text {disc }}^{2}(G(F) \backslash G(\mathbb{A}))$ of $L^{2}(G(F) \backslash G(\mathbb{A}))$ that decomposes discretely modulo the centre $Z=Z(G)$ of $G$. This distinction is only relevant to the reductive group $G L(N)$, in which $Z(F) \backslash Z(\mathbb{A})$ is noncompact.
} 
of their automorphic representations, which is compatible with their local decompositions (1.1), and depends only on the orbit of $\rho$ under the action of $\widehat{G}$ by conjugation on ${ }^{L} G$.

By correspondence we mean a relation rather than a function, and for this assertion, automorphic representations are to be understood in the inclusive sense of the precise definition in $[\mathbf{L} 3]$, which we have not given, rather than the restrictive characterization we have given. Langlands also stated a local form of the principle of functoriality, which applies to any local field $F$ of characteristic 0 . The compatibility assertion for global $F$ above is that if

$$
\pi^{\prime} \longrightarrow \pi
$$

then

$$
\pi_{v}^{\prime} \longrightarrow \pi_{v}
$$

for any completion $F_{v}$ of $F$. In other words, if $\pi^{\prime}$ and $\pi$ are automorphic representations of $G^{\prime}$ and $G$ that correspond under global functoriality for $\rho$, then their local components $\pi_{v}^{\prime}$ and $\pi_{v}$ correspond under local functoriality for the completion

$$
\rho_{v}:{ }^{L} G_{v}^{\prime} \longrightarrow{ }^{L} G_{v}
$$

of $\rho$.

This is the informal version of functoriality stated by Langlands in his original paper [L1]. It leaves unspecified the nature of the correspondence. Nevertheless, it still gives a sense of the depth of what was a completely new phenomenon. Whatever might be the internal relations among the constituents $\pi_{v}$ of the automorphic representation $\pi$ of $G$, they will be reflections of the corresponding internal relations among the constituents $\pi_{v}^{\prime}$ of the automorphic representation $\pi^{\prime}$ of $G^{\prime}$. This will become clearer when we describe more precise versions of functoriality in the final section of the paper.

The principle of functoriality is one of the great problems of mathematics. It has been established in a significant number of cases. However, these pale in comparison with the cases that remain unknown. Langlands has introduced some striking ideas for attacking the general case through a completely new application of the trace formula. (See $[\mathbf{F L N}],[\mathbf{L 6}]$ and $[\mathbf{A 3}$, Afterword]. It is not known, however, how far these ideas will ultimately take us. Even if they work in principle, there will still be many years of effort required by many mathematicians before they can be fully realized.

One of the goals of the monograph $[\mathbf{E C R}]$ was to establish the principle of functoriality in two basic cases. We shall describe them in turn.

The first case arises from the natural embedding of a complex classical group into a complex general linear group. Suppose that $G$ belongs to one of our families $\mathbf{B}_{n}, \mathbf{C}_{n}$ or $\mathbf{D}_{n}$. There is then a canonical embedding of the dual group $\widehat{G}$ into a general linear group $G L(N, \mathbb{C})$, for $N$ equal to $2 n, 2 n+1$ and $2 n$ respectively. If $G$ is split over $F$, this extends trivially to a canonical embedding

$$
{ }^{L} G=\widehat{G} \times \Gamma_{F} \longrightarrow{ }^{L}(G L(N))=G L(N, \mathbb{C}) \times \Gamma_{F}
$$

of the full $L$-group of $G$ to that of $G L(N)$. In the special case of type $\mathbf{C}_{n}$, we also obtain a nonstandard embedding of ${ }^{L} G$ into ${ }^{L}(G L(N))$ for any quadratic extension $E / F$, by mapping the quotient $\Gamma_{E / F}=\Gamma_{F} / \Gamma_{E}$ isomorphically into the central subgroup $\{ \pm 1\}$ of the image of $O(2 n+1, \mathbb{C})$ in $G L(N, \mathbb{C})$. If $G$ is not split over $F$, it 
is of type $\mathbf{D}_{n}$. The associated quadratic quotient $\Gamma_{E / F}$ then acts on $\widehat{G}=S O(2 n, \mathbb{C})$ through the nonidentity connected component of the complex group $O(2 n, \mathbb{C})$. This leads again to a canonical embedding

$$
{ }^{L} G=\widehat{G} \rtimes \Gamma_{F} \longrightarrow{ }^{L}(G L(N))=G L(N, \mathbb{C}) \times \Gamma_{F}
$$

of $L$-groups. We will discuss the various $L$-embeddings for this basic case of functoriality from a different perspective in Section 3.

In the second case, $G$ is above. This time, however, we take a product

$$
G^{\prime}=G_{1}^{\prime} \times G_{2}^{\prime}
$$

of smaller such groups. We require that the dual group

$$
\widehat{G}^{\prime}=\widehat{G}_{1}^{\prime} \times \widehat{G}_{2}^{\prime}
$$

come with a natural embedding into $\widehat{G}$. This means that

$$
\begin{aligned}
& \widehat{G}^{\prime}=S p(2 m, \mathbb{C}) \times S p(2 n-2 m, \mathbb{C}) \subset S p(2 n, \mathbb{C})=\widehat{G}, \\
& \widehat{G}^{\prime}=S O(2 m, \mathbb{C}) \times S O(2 n+1-2 m, \mathbb{C}) \subset S O(2 n+1, \mathbb{C})=\widehat{G},
\end{aligned}
$$

and

$$
\widehat{G}^{\prime}=S O(2 m, \mathbb{C}) \times S O(2 n-2 m, \mathbb{C}) \subset S O(2 n, \mathbb{C})=\widehat{G},
$$

for integers $0 \leq m \leq n$, where $G$ is of type $\mathbf{B}_{n}, \mathbf{C}_{n}$ and $\mathbf{D}_{n}$ respectively. If $G$ is of type $\mathbf{B}_{n}, G^{\prime}$ is split, and the embedding of ${ }^{L} G^{\prime}$ into ${ }^{L} G$ extends trivially to an $L$-embedding of ${ }^{L} G^{\prime}$ into ${ }^{L} G$. If $G$ is of type $\mathbf{C}_{n}, G$ and $G_{2}^{\prime}$ are split, but $G_{1}^{\prime}$ can be a quasisplit group defined by a quadratic extension $E_{1}$ of $F$. In this case, we obtain an $L$-embedding

$$
{ }^{L} G^{\prime}=\left(\widehat{G}_{1}^{\prime} \times \widehat{G}_{2}^{\prime}\right) \rtimes \Gamma_{F} \longrightarrow{ }^{L} G=\widehat{G} \times \Gamma_{F}
$$

from the nonstandard embedding of the second factor ${ }^{L} G_{2}^{\prime}$ attached to the quadratic extension $E_{1} / F$. Finally, if $G$ is of type $\mathbf{D}_{n}$, it is the quasisplit group defined by an extension $E=F(\sqrt{d})$ of degree 1 or 2 . We can then take $G_{1}^{\prime}$ and $G_{2}^{\prime}$ to be quasisplit groups of types $D_{m}$ and $D_{n-m}$ defined by any quadratic extensions $E_{1}=F\left(\sqrt{d_{1}}\right)$ and $E_{2}=F\left(\sqrt{d_{2}}\right)$ such that $d_{1} d_{2}$ equals $d$. It is then easy to see that there is a canonical embedding

$$
{ }^{L} G^{\prime}=\left(\widehat{G}_{1}^{\prime} \times \widehat{G}_{2}^{\prime}\right) \rtimes \Gamma_{F} \longrightarrow{ }^{L} G=\widehat{G} \rtimes \Gamma_{F}
$$

of $L$-groups.

We thus obtain two basic cases of the principle of functoriality by taking the $L$-homomorphism $\rho$ of Principle 1.1 to be any one of the $L$-embeddings we have just described. The first is at the heart of the classification of representations of $G$ (both local and global) in terms of those of $G L(N)$. The second provides the foundation for an understanding of the precise functorial correspondence from $G$ to $G L(N)$.

\section{The automorphic Langlands group $L_{F}$}

About ten years after formulating the principle of functoriality [L1], Langlands introduced something just as surprising $[\mathbf{L 4}]$. It is a hypothetical group that would be universal, in the sense that it could be used to characterize the automorphic representations of any group (connected and reductive, but not necessarily belonging to one of our four families) over a number field $F$. The existence of the Langlands 
group is closely related to the principle of functoriality, even somewhat deeper, but its hypothetical properties are still very useful for guidance and motivation.

In the earlier paper $[\mathbf{L} \mathbf{1}]$, Langlands had already predicted a major role for a smaller group, the variant of the absolute Galois group $\Gamma_{F}$ introduced by Weil. The Weil group $W_{F}$ is a locally compact group that is defined if $F$ is either local or global. It comes with a continuous homomorphism

$$
\phi: W_{F} \longrightarrow \Gamma_{F},
$$

with dense image and connected kernel, under which the preimage of any subgroup $\Gamma_{E}$ of $\Gamma_{F}$ of finite index equals the Weil group $W_{E}$ of the associated finite field extension $E$ of $F$. For each such $E, W_{F}$ is equipped also with a topological isomorphism

$$
r_{E}: C_{E} \longrightarrow W_{E}^{\mathrm{ab}}
$$

where $W_{E}^{\mathrm{ab}}=W_{E} / W_{E}^{c}$ is the abelianization of $W_{E}$, and

$$
C_{E}=\left\{\begin{array}{l}
E^{*}, \text { the multiplicative group of } E, \text { if } E \text { is local, } \\
\mathbb{A}_{E}^{*} / E^{*}, \text { the idèle class group of } E, \text { if } E \text { is global. }
\end{array}\right.
$$

The triplet $\left(W_{F}, \phi,\left\{r_{E}\right\}\right)$, which one usually denotes simply by $W_{F}$, is subject to four natural conditions $\left[\mathbf{T}, \S 1.1,\left(W_{1}\right)-\left(W_{4}\right)\right]$. With these conditions, $W_{F}$ is defined and uniquely determined up to an isomorphism, which itself is uniquely determined up to conjugation by an element in the kernel of $\phi$. (See $[\mathbf{T}]$.)

If $F=\mathbb{C}$, for example, $W_{F}$ equals the multiplicative group $\mathbb{C}^{*}$. If $F=\mathbb{R}, W_{F}$ is a nontrivial central extension

$$
1 \longrightarrow W_{\mathbb{C}} \longrightarrow W_{\mathbb{R}} \longrightarrow \mathbb{Z} / 2 \mathbb{Z} \longrightarrow 1
$$

of $W_{\mathbb{C}}$. It can be identified with the explicit group generated by $\mathbb{C}^{*}$ and a symbol $\sigma_{\mathbb{R}}$ with $\sigma_{\mathbb{R}}^{2}=-1$, subject to the conjugation relation

$$
\sigma_{\mathbb{R}} z \sigma_{\mathbb{R}}=\bar{z}, \quad z \in \mathbb{C}^{*} .
$$

If $F$ is a local field that is nonarchimedean (which is to say that it is not equal to $\mathbb{C}$ or $\mathbb{R}), W_{F}$ is an extension

$$
1 \longrightarrow I_{F} \longrightarrow W_{F} \longrightarrow\langle\text { Frob }\rangle \longrightarrow 1
$$

of an infinite cyclic group by a compact subgroup $I_{F}$ (the inertia group) of $\Gamma_{F}$. The element Frob represents the Frobenius automorphism in the absolute Galois group

$$
\operatorname{Gal}(\bar{k} / k) \cong \Gamma_{F} / I_{F}
$$

of the residue field $k$ of $F$, a compact, totally disconnected group, in which the cyclic group generated by Frob is dense. In this case, $W_{F}$ is still a reasonably explicit object. If $F$ is global, $W_{F}$ is more complicated. But as in the local case, the computations with $W_{F}$ that arise in representation theory can often be made quite explicit.

The global Weil group has some extra structure. It comes with embeddings of the local Weil groups $W_{F_{v}}$, which are determined up to conjugacy, and are compatible with the associated embeddings of Galois groups. In other words, for 
every valuation $v$ on $F$, we have a commutative diagram

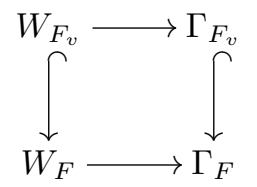

in which the vertical embeddings are determined up to conjugacy in $W_{F}$ and $\Gamma_{F}$.

Suppose that $G$ is one of our groups over $F$. Since

$$
W_{F} / W_{E} \cong \Gamma_{F} / \Gamma_{E}
$$

for any finite extension $E$ of $F$, the Weil group acts on $\widehat{G}$. We can therefore take the Weil form

$$
{ }^{L} G=\widehat{G} \rtimes W_{F}
$$

of the $L$-group. This seems more cumbersome than the Galois form, especially if we take the minimal Galois form with $\Gamma_{F}$ replaced by the finite group $\Gamma_{E / F}$. However, it is ultimately the best version of the $L$-group to work with. We can still talk about $L$-homomorphisms in this context, and we are free to formulate the principle of functoriality as in $\S 1$. We shall use the Weil form of the $L$-group in the rest of this section, as well as in the next.

Langlands conjectured the existence of a natural mapping $\phi \rightarrow \Pi_{\phi}$, from $L$ homomorphisms

$$
\phi: W_{F} \longrightarrow{ }^{L} G,
$$

taken up to conjugacy of ${ }^{L} G$ by its subgroup $\widehat{G}$, to packets $\Pi_{\phi}$ of irreducible representations. The proposed sets $\Pi_{\phi}$ later become known as $L$-packets, since they were conjectured to consist of representations with the same $L$-functions and $\varepsilon$-factors. For local $F$, they should be finite subsets of $\Pi(G)$. For global $F$, they should be compatible with the localizations $\phi \rightarrow \phi_{v}$ defined by the embeddings (2.1). More precisely, the global $L$-packet of a global parameter $\phi$ would simply be defined as the set

$$
\Pi_{\phi}=\left\{\pi=\widetilde{\bigotimes}_{v} \pi_{v}: \pi_{v} \in \Pi_{\phi_{v}}, \pi_{v} \text { unramified }^{4} \text { for almost all } v\right\}
$$

of irreducible representations of $G(\mathbb{A})$. The conjectural part of this global definition can be taken as an assertion that the global $L$-functions attached to representations $\pi \in \Pi_{\phi}$ have analytic continuation and functional equation. This conjecture, local and global, became known as the Langlands correspondence. Notice that $W_{F}$ can be regarded as the Weil form of the $L$-group ${ }^{L} G^{\prime}$, for the trivial group $G^{\prime}=\{1\}$ over $F$. The Langlands correspondence can therefore be regarded as a very special case of principle of functoriality. It is an illustration of the depth of functoriality, as a conjecture that governs the very foundations of the subject in spite of its seemingly innocuous statement.

If $F$ equals $\mathbb{C}$ or $\mathbb{R}$, Langlands established a correspondence $\phi \rightarrow \Pi_{\phi}$ for any $G$ over $F$. Part of the problem was of course to formulate the definitions, which necessarily go to the roots of Harish-Chandra's monumental contributions to harmonic analysis on real groups. Langlands then showed that $\Pi(G)$ is a disjoint union of the packets $\Pi_{\phi}$, taken over the ( $\widehat{G}$-orbits) of $L$-homomorphisms $\phi$. His results can be regarded as a classification of the representations of a real group.

\footnotetext{
${ }^{4}$ We will leave this notion undefined for the present, and return to it in $\S 4$.
} 
If $F$ is local nonarchimedean, much less is known, in part because there is no $p$-adic analogue of Harish-Chandra's classification of the discrete series. It was also clear from early examples that the $L$-packets $\Pi_{\phi}$ would not exhaust the representations in $\Pi(G)$. If $F$ is global, the problem not surprisingly is much deeper. In particular, it is known that the automorphic representations $\pi \in \Pi(G)$ that happen to lie in some Weil packet $\Pi_{\phi}$ are really quite sparse. It was with this understanding that Langlands was led to introduce a larger group that would replace $W_{F}$ in his conjectural correspondence.

Assume that $F$ is global. In Langlands' original article $[\mathbf{L 4}]$, the universal automorphic Galois group was to be an object in the category of complex, reductive pro-algebraic groups. It was formulated as an extension of the absolute Galois group $\Gamma_{F}$ by a connected, complex, reductive pro-algebraic group. Kottwitz $[\mathbf{K}]$ later pointed out that the group would be simpler if it were taken in the category of locally compact topological groups, like the Weil group itself. In this formulation, the global Langlands group $L_{F}$ should be an extension of $W_{F}$ by a connected, compact group. It would thus take its place in a sequence

$$
L_{F} \longrightarrow W_{F} \longrightarrow \Gamma_{F}
$$

of three locally compact groups, all having fundamental ties to the arithmetic of $F$. The group $L_{F}$ should also have a local analogue $L_{F_{v}}$, for any valuation $v$ on $F$, that embeds into an extension

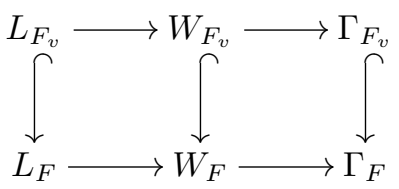

of the commutative diagram (2.1). In particular, $L_{F}$ should be equipped with a canonical embedding of $L_{F_{v}}$, which as in the cases of $W_{F}$ and $\Gamma_{F}$, is determined only up to conjugacy.

Suppose that $F$ is local. The local Langlands group $L_{F}$ is known. It is defined as

$$
L_{F}= \begin{cases}W_{F}, & \text { if } F \text { is archimedean, } \\ W_{F} \times S U(2), & \text { if } F \text { is nonarchimedean. }\end{cases}
$$

Given $G$ over $F$, we write $\Phi(G)$ for the set of $L$-homomorphisms

$$
\phi: L_{F} \longrightarrow{ }^{L} G,
$$

taken up to $\widehat{G}$-conjugacy. The conjectural Langlands correspondence for $G$ again takes the form of a mapping

$$
\phi \longrightarrow \Pi_{\phi}, \quad \phi \in \Phi(G),
$$

from $\Phi(G)$ to finite subsets $\Pi_{\phi}$ of $\Pi(G)$. However, this time it should have the property

$$
\Pi(G)=\coprod_{\phi \in \Phi(G)} \Pi_{\phi}
$$

of exhaustion. In particular, the extra unitary factor $S U(2)$ in the definition (2.4) would be what is needed to obtain a full classification in case $F$ is $p$-adic. It is equivalent to the supplementary data that had been used earlier to construct the Weil-Deligne group. (See $[\mathbf{T}],[\mathbf{L} \mathbf{4}]$.) 
Suppose again that $F$ is global. The global Langlands group $L_{F}$ is far from known. It would be much larger than $W_{F}$, for the reason that "most" automorphic representations contain information that cannot be reduced to something as simple as the Weil group. In the article [A2], we described a conjectural construction of $L_{F}$. It is given by an extension

$$
1 \longrightarrow K_{F} \longrightarrow L_{F} \longrightarrow W_{F} \longrightarrow 1
$$

of $W_{F}$ by an infinite product $K_{F}$ of compact, connected, simply connected groups. The factors of $K_{F}$ are parametrized by certain very basic automorphic representations of $G$ (which we called primitive in $[\mathbf{A 2}]$ ), as $G$ ranges over all simply connected, quasisplit groups. The group $L_{F}$ would then be somewhat self-referential, in that it is supposed to classify automorphic representations, and yet at the same time, is built out of certain automorphic representations. This does not make its existence any easier to establish. One requires the principle of functoriality for all groups $G$, and more, to define the primitive automorphic representations at the core of $L_{F}$.

In any case, the global Langlands group is supposed to be characterized in terms of automorphic representations of general linear groups. It was predicated by Langlands on the assumption that for any $N$, there is a bijective correspondence between irreducible $N$-dimensional representations of $L_{F}$, and cuspidal automorphic representations of the group $G L(N)$. We recall that the cuspidal automorphic representations are the fundamental building blocks for general automorphic representations, and always occur in the discrete spectrum. (See [BJ, p. 191-197] and [L3, Proposition 2].) More generally, given one of our groups $G$ over $F$, and assuming the existence of the global group $L_{F}$, we define the associated set of $L$ homomorphisms $\Phi(G)$ as in the local case (2.5). The elements $\phi \in \Phi(G)$ would again parametrize global $L$-packets $\Pi_{\phi}$, defined in terms of their local components by (2.2). As in the special case of a Weil parameter, we could state the conjectural part of this global definition by saying that global $L$-functions attached to representations $\pi \in \Pi_{\phi}$ should have analytic continuation and functional equation.

In terms of representation theory, the real global conjecture concerns automorphic representations in a packet $\Pi_{\phi}$. It is convenient to write $\Phi_{\text {bdd }}(G)$ (in both the local and global cases) for the subset of parameters in $\Phi(G)$ whose image in ${ }^{L} G$ projects onto a bounded (that is, relatively compact) subset of $\widehat{G}$. If $G=G L(N)$, for example, elements in $\Phi_{\text {bdd }}(G)$ project to $N$-dimensional representations of $L_{F}$ that are unitary. With $F$ being global, there will be no automorphic representations $\pi$ in the packet $\Pi_{\phi}$ of any global parameter in the complement of $\Phi_{\text {bdd }}(G)$. This is only because we have taken the restricted definition of automorphic representations, as elements $\pi \in \Pi(G)$ that occur in the automorphic spectral decomposition, rather than including the supplementary representations obtained by analytic continuation into the complex domain, as in [L3]. By the same token, if $F$ is local, there will be no tempered representations $\pi$ in the packet of a local parameter $\phi$ taken from the complement of $\Phi_{\text {bdd }}(G)$.

If $F$ is global, the packets $\Pi_{\phi}$ of parameters $\phi \in \Phi_{\mathrm{bdd}}(G)$ should contain many automorphic representations $\pi$. However, they will still not exhaust the set $\Pi(G)$ of all automorphic representations. The situation here is like that of a nonarchimedean local field $F$, where we had to define local parameters on the product $W_{F} \times S U(2)$ rather than just $W_{F}$. The final step for the global field $F$ is likewise to add a factor $S U(2)$, and form a product $L_{F} \times S U(2)$. The localization of this group at $v$, which 
will be needed to construct the local components of representations attached to the resulting global parameters, will be a new product $L_{F_{v}} \times S U(2)$. In particular, if $F_{v}$ is nonarchimedean, we will be dealing with a local product

$$
W_{F_{v}} \times S U(2) \times S U(2)
$$

with two factors $S U(2)$.

If $F$ is local or global, we write $\Psi(G)$ for the set of $L$-homomorphisms

$$
\psi: L_{F} \times S U(2) \longrightarrow{ }^{L} G,
$$

taken up to $\widehat{G}$-conjugacy, but with the property that the restriction of $\psi$ to $L_{F}$ lies in the subset $\Phi_{\text {bdd }}(G)$ of $\Phi(G)$. If $F$ is global, the diagram (2.3) gives a localization mapping ${ }^{5}$

$$
\psi \longrightarrow \psi_{v}
$$

from $\Psi(G)$ to $\Psi\left(G_{v}\right)$. If $F$ is local, we conjecture that there is again a natural mapping $\psi \rightarrow \Pi_{\psi}$, this time from parameters $\psi \in \Psi(G)$ to finite subsets $\Pi_{\psi}$ of $\Pi_{\text {unit }}(G)$. If $F$ is global, we will then be able to attach a global packet

$$
\Pi_{\psi}=\left\{\pi=\tilde{\bigotimes}_{v} \pi_{v}: \pi_{v} \in \Pi_{\pi_{v}}, \pi_{v} \text { unramified for almost all } v\right\}
$$

of irreducible unitary representations of $G(\mathbb{A})$ to parameters $\psi \in \Psi(G)$. Many of the representations in these packets should be automorphic. Moreover, they ought now to exhaust the set of all automorphic representations.

The set $\Psi(G)$, defined for $F$ local or global, is where the process ends. It contains $\Phi_{\text {bdd }}(G)$, as the set of parameters on $L_{F} \times S U(2)$ that vanish on the second factor. The set $\Phi_{\text {bdd }}(G)$ in turn contains the set $\Phi_{\text {bdd }}^{W}(G)$ of bounded parameters on $W_{F}$, as the parameters on $L_{F}$ that are pullbacks from $W_{F}$. We obtain embeddings

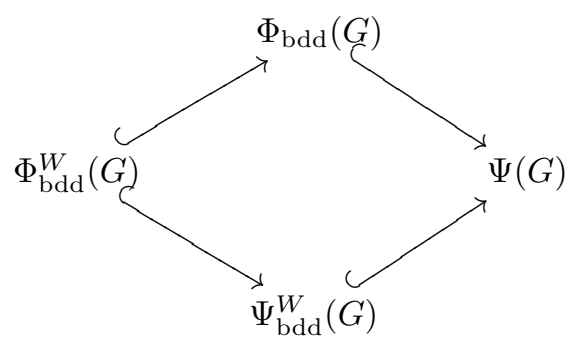

where $\Phi_{\mathrm{bdd}}^{W}(G)$ is the subset of parameters in $\Psi(G)$ whose restrictions to $L_{F}$ lie in the subset $\Phi_{\text {bdd }}^{W}(G)$. The global family $\Psi(G)$ appears to be the right set of parameters for classifying automorphic representations of $G$. Its localizations are then forced on us if we want to describe the local constituents of automorphic representations.

There is still something more to say. We would of course like to know how to construct the local packets $\Pi_{\psi}$. We would also like to characterize which representations in a global packet $\Pi_{\psi}$ are actually automorphic. The answers to these questions are closely related to a certain finite group attached to $\psi$.

\footnotetext{
${ }^{5}$ We are assuming in this heuristic discussion that the generalized Ramanujan conjecture is valid for $G L(N)$. Since this is not known, one must make a minor adjustment in practice. See the discussion of [ECR, (1.3.10)] and of (4.12) in $\S 4$ here.
} 
For any $\psi \in \Psi(G)$, with $F$ being either local or global, we have the centralizer

$$
S_{\psi}=\operatorname{Cent}(\operatorname{im}(\psi), \widehat{G})
$$

of the image of $\psi$ in $\widehat{G}$, a complex, reductive subgroup of $\widehat{G}$. We also have its finite group

$$
\mathcal{S}_{\psi}=\bar{S}_{\psi} / \bar{S}_{\psi}^{0}=S_{\psi} / S_{\psi}^{0} Z(\widehat{G})^{\Gamma_{F}}
$$

of connected components, or rather, connected components in the quotient

$$
\bar{S}_{\psi}=S_{\psi} / Z(\widehat{G})^{\Gamma_{F}}
$$

of $S_{\psi}$ by the group of elements in the centre $Z(\widehat{G})$ of $\widehat{G}$ that are invariant under the action of the Galois group $\Gamma_{F}$. (The group $Z(\widehat{G})^{\Gamma_{F}}$ equals $Z(\widehat{G})$ unless $G$ is a nonsplit group $S O(2)$, and in particular, of type $D_{n}$ with $n=1$.)

With our standing agreement that $G$ belongs to one of the families $\mathbf{A}_{n}-\mathbf{D}_{n}$, the quotient $\mathcal{S}_{\psi}$ is an abelian 2-group. If $F$ is local, the representations in the local packet $\Pi_{\psi}$ are closely tied to the group of (linear) characters on $\mathcal{S}_{\psi}$. If $F$ is global, the group $S_{\psi}$ is contained in the centralizer $S_{\psi_{v}}$ of any localization $\psi_{v}$. It follows that there is a canonical mapping from $\mathcal{S}_{\psi}$ into $\mathcal{S}_{\psi_{v}}$. The representations in a global packet $\Pi_{\psi}$ that are automorphic are then tied to characters on the product

$$
\mathcal{S}_{\psi, \mathbb{A}}=\prod_{v} \mathcal{S}_{\psi_{v}}
$$

whose restrictions to the diagonal image of $\mathcal{S}_{\psi}$ equal a certain character $\varepsilon_{\psi}$ on $\mathcal{S}_{\psi}$ that is defined explicitly in terms of arithmetic invariants attached to $\psi$. The case that $\varepsilon_{\psi}$ is nontrivial will obviously be interesting, as also will be the case that the mapping from $\mathcal{S}_{\psi}$ to $\mathcal{S}_{\psi, \mathbb{A}}$ is not injective. But if neither of these exceptional conditions hold for $\psi$, we see a particularly clear analogy between the local groups $G\left(F_{v}\right)$ and global quotient $G(F) \backslash G(\mathbb{A})$ introduced in $\S 1$ and the "miniature models" $\mathcal{S}_{\psi_{v}}$ and $\mathcal{S}_{\psi} \backslash \mathcal{S}_{\psi, \mathbb{A}}$ we have just defined.

\section{Self-dual, finite dimensional representations}

The last section contained a good deal of information, some of which is perhaps difficult to absorb at the early stages of our presentation. Moreover, it seems to have been built on the shaky foundation of a hypothetical group $L_{F}$. Some such discussion, however, is a necessary part of the exposition. It helps us to see what we should be looking for.

We are trying to classify representations of groups $G$ of type $\mathbf{B}_{n}, \mathbf{C}_{n}$ and $\mathbf{D}_{n}$ in terms of those of general linear groups $G L(N)$. If representations are indeed attached to parameters on a group $L_{F} \times S U(2)$, it makes sense to try to compare parameters for $G$ with those of $G L(N)$. We shall do so in this section. The calculation will be an elementary exercise in linear algebra, which follows the discussion of $\S 1.2$ of $[\mathbf{E C R}]$.

We are assuming that $F$ is a local or global field of characteristic 0. Suppose that $\Lambda_{F}$ is any locally compact group over $W_{F}$ with connected kernel. We are of course thinking of the case that $\Lambda_{F}$ equals the product $L_{F} \times S U(2)$ (which is hypothetical if $F$ is global), but the arguments will be the same for any $\Lambda_{F}$. We have already noted the obvious fact that an $L$-homomorphism from $\Lambda_{F}$ to the $L$-group of $G L(N)$ 
projects onto the dual group $G L(N, \mathbb{C})$ of $G L(N)$, and can therefore be identified with an $N$-dimensional (continuous, complex) representation

$$
r: \Lambda_{F} \longrightarrow G L(N, \mathbb{C})
$$

of $\Lambda_{F}$.

We say that $r$ is self-dual if it is equivalent to its contragredient representation

$$
r^{\vee}(\lambda)={ }^{t} r(\lambda)^{-1}, \quad \lambda \in \Lambda_{F},
$$

where $x \rightarrow{ }^{t} x$ is the usual transpose mapping. In other words, the equivalence class of $r$ is invariant under the usual automorphism

$$
\theta(x)=x^{\vee}={ }^{t} x^{-1}, \quad x \in G L(N),
$$

of $G L(N)$. This condition depends only on the inner class of $\theta$. It remains the same if $\theta$ is replaced by any conjugate

$$
\theta_{g}(x)=g^{-1} \theta(x) g, \quad g \in G L(N) .
$$

We shall analyze the self-dual representations $r$ in terms of orthogonal and symplectic subgroups of $G L(N, \mathbb{C})$.

We decompose a given representation $r$ into a direct sum

$$
r=\ell_{1} r_{1} \oplus \cdots \oplus \ell_{r} r_{r},
$$

for inequivalent representations

$$
r_{k}: \Lambda_{F} \longrightarrow G L\left(N_{k}, \mathbb{C}\right), \quad 1 \leq k \leq r,
$$

and multiplicities $\ell_{k}$ with

$$
N=\ell_{1} N_{1}+\cdots+\ell_{r} N_{r} .
$$

The representation is self-dual if and only if there is an involution $k \rightarrow k^{\vee}$ on the indices such that for any $k, r_{k}^{\vee}$ is equivalent to $r_{k^{\vee}}$ and $\ell_{k}=\ell_{k^{\vee}}$. We say that $r$ is elliptic if it satisfies the further constraint that for each $k, k^{\vee}=k$ and $\ell_{k}=1$. We shall concentrate on this case.

Assume that $r$ is elliptic. Then

$$
r=r_{1} \oplus \cdots \oplus r_{r},
$$

for distinct, irreducible, self-dual representations $r_{i}$ of $\Lambda_{F}$ of degree $N_{i}$. If $i$ is any index, we can write

$$
r_{i}^{\vee}(\lambda)=A_{i} r_{i}(\lambda) A_{i}^{-1}, \quad \lambda \in \Lambda_{F},
$$

for a fixed element $A_{i} \in G L\left(N_{i}, \mathbb{C}\right)$. Applying the automorphism $\theta$ to each side of this equation, we see that

$$
r_{i}(\lambda)=A_{i}^{\vee} r_{i}^{\vee}(\lambda)\left(A_{i}^{\vee}\right)^{-1}=\left(A_{i}^{\vee} A_{i}\right) r_{i}(\lambda)\left(A_{i}^{\vee} A_{i}\right)^{-1} .
$$

Since $r_{i}$ is irreducible, the product $A_{i}^{\vee} A_{i}$ is a scalar matrix. We can therefore write

$$
{ }^{t} A_{i}=c_{i} A_{i}, \quad c_{i} \in \mathbb{C}^{*} .
$$

If we take the transpose of each side of this equation, we see further that $c_{i}^{2}=1$. Thus, $c_{i}$ equals +1 or -1 , and the nonsingular matrix $A_{i}$ is either symmetric or skew-symmetric. The mapping

$$
x_{i} \longrightarrow\left(A_{i}^{-1}\right)^{t} x_{i} A_{i}, \quad x_{i} \in G L\left(N_{i}\right),
$$


represents the adjoint relative to the bilinear form defined by $A_{i}$. Therefore $r_{i}(\lambda)$ belongs to the corresponding orthogonal group $O\left(A_{i}, \mathbb{C}\right)$ or symplectic group $\operatorname{Sp}\left(A_{i}, \mathbb{C}\right)$, according to whether $c_{i}$ equals +1 or -1 .

Let us write $I_{O}$ and $I_{S}$ for the set of indices $i$ such that $c_{i}$ equals +1 and -1 respectively. We then write

$$
\begin{aligned}
r_{\varepsilon}(\lambda) & =\bigoplus_{i \in I_{\varepsilon}} r_{i}(\lambda), \quad \lambda \in \Lambda_{F}, \\
A_{\varepsilon} & =\bigoplus_{i \in I_{\varepsilon}} A_{i},
\end{aligned}
$$

and

$$
N_{\varepsilon}=\sum_{i \in I_{\varepsilon}} N_{i},
$$

for a symbol $\varepsilon$ that can be either $O$ or $S$. Thus $A_{O}$ is a symmetric matrix in $G L\left(N_{O}, \mathbb{C}\right), A_{S}$ is a skew-symmetric matrix in $G L\left(N_{S}, \mathbb{C}\right)$, and $r_{O}$ and $r_{S}$ are representations of $\Lambda_{F}$ that take values in the respective groups $O\left(A_{O}, \mathbb{C}\right)$ and $S p\left(A_{S}, \mathbb{C}\right)$. We have established a canonical decomposition

$$
r=r_{O} \oplus r_{S}
$$

of the self-dual representation $r$ into orthogonal and symplectic components.

It is only the equivalence class of $r$ that is relevant. We can therefore replace $r(\lambda)$ with its conjugate by any matrix $B \in G L(N, \mathbb{C})$. This has the effect of replacing the matrix

$$
A=A_{O} \oplus A_{S}
$$

in $G L(N, \mathbb{C})$ by ${ }^{t} B A B$. In particular, we can take $A_{O}$ to be any symmetric matrix in $G L\left(N_{O}, \mathbb{C}\right)$ and $A_{S}$ to be any skew-symmetric matrix in $G L\left(N_{S}, \mathbb{C}\right)$. We can therefore put the orthogonal and symplectic groups that contain the images of $r_{O}$ and $r_{S}$ into some standard form.

It is generally most convenient to choose the standard form for these groups so that the subgroup of diagonal matrices is a maximal torus. For our standard orthogonal group in $G L(N)$, we therefore take

$$
O(N)=O(J),
$$

where

$$
J=J(N)=\left(\begin{array}{lll}
0 & & 1 \\
& . & \\
1 & & 0
\end{array}\right)
$$

is the second diagonal in $G L(N)$. It is a group with two connected components, whose identity component is the special orthogonal group

$$
S O(N)=\{x \in O(N): \operatorname{det}(x)=1\} .
$$

As the standard symplectic group in $G L(N)$, defined for $N$ even, we can take

$$
S p(N)=S p(\widetilde{J}),
$$


where

$$
\widetilde{J}=\widetilde{J}(N)=\left(\begin{array}{cccc}
0 & & & 1 \\
& & -1 & \\
(-1)^{N+1} & & & 0
\end{array}\right)
$$

for any $N$. Notice that $S p(N)$ is the group of fixed points of the automorphism

$$
\widetilde{\theta}(N)=\operatorname{Int}(\widetilde{J}) \circ \theta: x \longrightarrow \widetilde{J} \theta(x) \widetilde{J}^{-1}
$$

while $O(N)$ is the group of fixed points of the automorphism

$$
\operatorname{Int}(\widetilde{I}) \circ \widetilde{\theta}(N)
$$

in the inner class of $\widetilde{\theta}(N)$ (and $\theta$ ), where

$$
\widetilde{I}=\widetilde{I}(N)=J \widetilde{J}^{-1}=\left(\begin{array}{cccc}
1 & & & 0 \\
& -1 & & \\
& & \ddots & \\
0 & & & (-1)^{N+1}
\end{array}\right) .
$$

It is customary to treat $\widetilde{\theta}(N)$ as the standard automorphism in the inner class of $\theta$, since it stabilizes the standard splitting $[\mathbf{K},(1.3)]$ of $G L(N)$.

Returning to our discussion, we can arrange that $A$ equals $J\left(N_{O}\right) \oplus \widetilde{J}\left(N_{S}\right)$. In the interest of symmetry, we actually take $A$ to be the matrix

$$
J_{O, S}=J\left(N_{O}, N_{S}\right)=\left(\begin{array}{ccc}
0 & & \widetilde{J}\left(N_{S}^{\prime}\right) \\
\widetilde{J}\left(N_{S}^{\prime}\right) & J\left(N_{O}\right) &
\end{array}\right), \quad N_{S}=2 N_{S}^{\prime},
$$

obtained from the obvious embedding of $J\left(N_{O}\right) \oplus \widetilde{J}\left(N_{S}\right)$ into $G L(N, \mathbb{C})$. The associated representation $r$ from the given equivalence class then maps $\Lambda_{F}$ to the corresponding subgroup of $G L(N, \mathbb{C})$, namely the subgroup

$$
O\left(N_{O}, \mathbb{C}\right) \times S p\left(N_{S}, \mathbb{C}\right)
$$

defined by the embedding

$$
(x, y) \longrightarrow\left(\begin{array}{ccc}
y_{11} & 0 & y_{12} \\
0 & x & 0 \\
y_{21} & 0 & y_{22}
\end{array}\right)
$$

where $y_{i j}$ are the four $\left(N_{S}^{\prime} \times N_{S}^{\prime}\right)$-block components of the matrix $y \in S p\left(N_{S}, \mathbb{C}\right)$.

The subrepresentations $r_{O}$ and $r_{S}$ of $r$ can be analyzed separately. The symplectic factor $r_{S}$ is the simpler of the two. Its image is contained in the connected complex group

$$
\widehat{G}_{S}=S p\left(N_{S}, \mathbb{C}\right) .
$$

This in turn is the dual group of the split special orthogonal group

$$
G_{S}=S O\left(N_{S}+1\right) .
$$

We need say nothing more in this case.

The orthogonal factor $r_{O}$ is complicated by the fact that its image is contained in the disconnected group $O\left(N_{O}, \mathbb{C}\right)$. Its projection

$$
\Lambda_{F} \longrightarrow O\left(N_{O}, \mathbb{C}\right) / S O\left(N_{O}, \mathbb{C}\right)=\mathbb{Z} / 2 \mathbb{Z}
$$


onto the corresponding group of connected components is a character $\eta$ of $\Lambda_{F}$ of order 1 or 2 . Since the kernels of the mappings $\Lambda_{F} \rightarrow W_{F}$ and $W_{F} \rightarrow \Gamma_{F}$ are connected, $\eta$ can be identified with a character on the Galois group $\Gamma_{F}$ of order 1 or 2. This determines a field extension $E$ of $F$ of degree 1 or 2 .

Suppose first that $N_{O}$ is odd. In this case, the matrix $(-I)$ in $O\left(N_{O}\right)$ belongs to the nonidentity component, and the orthogonal group is a direct product

$$
O\left(N_{O}, \mathbb{C}\right)=S O\left(N_{O}, \mathbb{C}\right) \times \mathbb{Z} / 2 \mathbb{Z}
$$

We write

$$
S O\left(N_{O}, \mathbb{C}\right)=\widehat{G}_{O}
$$

where $G_{O}$ is the split group $S p\left(N_{O}-1\right)$ over $F$. We then use $\eta$ to identify the direct product

$$
{ }^{L} G_{O, E / F}=\widehat{G}_{O} \times \Gamma_{E / F}
$$

with a subgroup of $O\left(N_{O}, \mathbb{C}\right)$, namely $S O\left(N_{O}, \mathbb{C}\right)$ or $O\left(N_{O}, \mathbb{C}\right)$, according to whether $\eta$ has order 1 or 2 . We thus have an embedding of a restricted form ${ }^{L} G_{O, E / F}$ of the $L$-group of $G_{O}$ into $G L\left(N_{O}, \mathbb{C}\right)$.

Assume next that $N_{O}$ is even. In this case, the nonidentity component in $O\left(N_{O}\right)$ acts by an outer automorphism on $S O\left(N_{O}\right)$. We write

$$
S O\left(N_{O}, \mathbb{C}\right)=\widehat{G}_{O}
$$

where $G_{O}$ is now the corresponding quasisplit orthogonal group $S O\left(N_{O}, \eta\right)$ over $F$ defined by $\eta$. In other words, $G_{O}$ is the split group $S O\left(N_{O}\right)$ if $\eta$ is trivial, and the nonsplit group obtained by twisting $S O\left(N_{O}\right)$ over $E$ by the given outer automorphism if $\eta$ is nontrivial. Let $\widetilde{\omega}\left(N_{O}\right)$ be the permutation matrix in $G L\left(N_{O}\right)$ that interchanges the middle two co-ordinates, and leaves the other co-ordinates invariant. We take this element as a representative of the nonidentity component of $O\left(N_{O}, \mathbb{C}\right)$. We then use $\eta$ to identify the semidirect product

$$
{ }^{L} G_{O, E / F}=\widehat{G} \rtimes \Gamma_{E / F}
$$

with a subgroup of $O\left(N_{O}, \mathbb{C}\right)$, namely $S O\left(N_{O}, \mathbb{C}\right)$ or $O\left(N_{O}, \mathbb{C}\right)$ as before. We again obtain an embedding of a restricted (in this case minimal) $L$-group of $G_{O}$ into $G L\left(N_{O}, \mathbb{C}\right)$.

We have shown that the elliptic self-dual representation $r$ factors through the embedded subgroup

$$
{ }^{L} G_{E / F}={ }^{L}\left(G_{O} \times G_{S}\right)_{E / F}
$$

of $G L(N, \mathbb{C})$ attached to the quasisplit group

$$
G=G_{O} \times G_{S}
$$

over $F$. The group $G$ is called a twisted endoscopic group for $G L(N)$. It is determined by $r$, and in fact, by the decomposition $N=N_{O}+N_{S}$ and the character $\eta=\eta_{G}$ attached to $r$. The same is true of the $L$-embedding

$$
\xi=\xi_{O, S, \eta}:{ }^{L} G=\widehat{G} \rtimes \Gamma_{F} \longleftrightarrow{ }^{L}(G L(N))=G L(N, \mathbb{C}) \times \Gamma_{F},
$$

obtained by inflating the embedding above to the full $L$-groups. A third object we can associate to the decomposition $N=N_{O}+N_{S}$, and hence to $r$, is the product

$$
s=s_{O, S}=J_{O, S}^{-1} \rtimes \theta .
$$

It is a semisimple element in the coset

$$
\widehat{\widetilde{G}}(N)=G L(N, \mathbb{C}) \rtimes \theta
$$


of $\theta$ in the semidirect product $\widehat{\widetilde{G}}(N)^{+}$of $G L(N, \mathbb{C})$ with the group $\theta^{+}$of order 2 generated by $\theta$. The complex group $\widehat{G}=\widehat{G}_{O} \times \widehat{G}_{S}$ is then the connected centralizer of $s$ in the subgroup

$$
G L(N, \mathbb{C})=G L(N, \mathbb{C}) \rtimes 1 .
$$

The triplet $(G, s, \xi)$ is called a twisted endoscopic datum for $G L(N)$.

The triplet $(G, s, \xi)$ belongs to a special class, called elliptic twisted endoscopic data. This is a consequence of our condition that the original self-dual representation is elliptic. A general (nonelliptic) twisted endoscopic datum for $G L(N)$ is again a triplet $(G, s, \xi)$, where $G$ is a quasisplit group over $F, s$ is a semisimple element in $G L(N, \mathbb{C}) \rtimes \theta$ whose connected centralizer in $G L(N, \mathbb{C})$ equals $\widehat{G}$, and $\xi$ is an $L$-embedding of ${ }^{L} G$ into ${ }^{L} G L(N)$. We require that $\xi$ equal the identity on $\widehat{G}$, and that the projection onto $G L(N, \mathbb{C})$ of the image of $\xi$ lie in the full centralizer of $s$. The twisted endoscopic group $G$ (or datum) is said to be elliptic if its subgroup $Z(\widehat{G})^{\Gamma_{F}}$ is finite.

We have been following a special case of the general terminology of [KS, p. 16]. The notion of isomorphism between general endoscopic data is defined in $[\mathbf{K S}$, p. 18]. In the case at hand, it is given by an element $g$ in the dual group $G L(N, \mathbb{C})$ whose action by conjugation is compatible (in a natural sense we do not spell out, but which is easy to imagine) with the two twisted endoscopic data. We write Aut $_{N}(G)$ for the group of isomorphisms of the twisted endoscopic datum $G$ to itself. The main role for this group is in its image

$$
\widetilde{O u t}_{N}(G)=\widetilde{\operatorname{Aut}_{N}}(G) / \widetilde{\operatorname{Int}}_{N}(G)
$$

in the group of outer automorphisms of the group $G$ over $F$. (Following standard practice, we often let the twisted endoscopic group $G$ represent a full datum $(G, s, \xi)$, or even an isomorphism class of data.) If $G$ represents one of the elliptic data above, Out $_{N}(G)$ is trivial if the integer $N_{O}$ is odd or zero. In the remaining case that $N_{O}$ is even and positive, $\widetilde{O u t}_{N}(G)$ is a group of order 2, the nontrivial element being the outer automorphism induced by the nontrivial component of $O\left(N_{O}, \mathbb{C}\right)$, which we have represented by the permutation matrix $\widetilde{\omega}\left(N_{O}\right)$ above.

We write $^{6}$

$$
\widetilde{\mathcal{E}}(N)=\mathcal{E}(\widetilde{G}(N))
$$

for the set of isomorphism classes of twisted endoscopic data for $G L(N)$, and

$$
\widetilde{\mathcal{E}}_{\mathrm{ell}}(N)=\mathcal{E}_{\mathrm{ell}}(\widetilde{G}(N))
$$

for the subset of classes that are elliptic. The data $(G, s, \xi)$, attached to equivalence classes of elliptic, self-dual representations $r$ as above, form a set of representative of $\widetilde{\mathcal{E}}_{\text {ell }}(N)$. The set $\widetilde{\mathcal{E}}_{\text {ell }}(N)$ is thus parametrized by triplets $\left(N_{O}, N_{S}, \eta\right)$, where $N_{O}+N_{S}=N$ is a decomposition of $N$ into nonnegative integers with $N_{S}$ even, and $\eta=\eta_{G}$ is a character of $\Gamma_{F}$ of order 1 or 2 such that $\eta=1$ if $N_{O}=0$ and $\eta \neq 1$ if $N_{O}=2$. (The last constraint is required in order that the datum be elliptic.)

Our general goal is to describe the representations of a quasisplit, special orthogonal or symplectic group in terms of those of general linear groups. The twisted endoscopic groups $G$ are obviously relevant to the problem. Indeed, they are just the

6 It is understood that $\widetilde{G}(N)=G L(N) \rtimes \theta$ is the nonidentity component of the reductive, nonconnected group $\widetilde{G}(N)^{+}=G L(N) \rtimes \theta^{+}$over $F$, and that the complex variety $\widehat{\widetilde{G}}(N)=G L(N, \mathbb{C}) \rtimes \theta$ above is the associated "dual set". 
groups we want to study. But they are also part of the broader theory of endoscopy, which can therefore be brought to bear on the task. Since the general arguments are often inductive, the case that $\widehat{G}$ is either purely orthogonal or purely symplectic will have a special role. Accordingly, we write

$$
\widetilde{\mathcal{E}}_{\text {sim }}(N)=\mathcal{E}_{\text {sim }}(\widetilde{G}(N))
$$

for the subset of elements in $\widetilde{\mathcal{E}}_{\text {ell }}(N)$ that are simple, in the sense that one of the integers $N_{O}$ or $N_{S}$ vanishes. We then have a chain of sets

$$
\widetilde{\mathcal{E}}_{\text {sim }}(N) \subset \widetilde{\mathcal{E}}_{\text {ell }}(N) \subset \widetilde{\mathcal{E}}(N),
$$

which are all finite if $F$ is local, and all infinite if $F$ is global.

The objects we have been discussing concern the $L$-embeddings for the first case of functoriality at the end of $\S 1$. We have provided some motivation for them. We have also used them as a way to introduce the theory of twisted endoscopy for $G L(N)$. We shall now consider the $L$-embeddings of the second case of functoriality from the end of $\S 1$.

To this end, we note that in addition to the twisted endoscopic data for $G L(N)$, one has also to work with ordinary (untwisted) endoscopic data. These are attached to our quasisplit, special orthogonal or symplectic groups, which is to say, the groups $G \in \widetilde{\mathcal{E}}_{\text {sim }}(N)$. An endoscopic datum for $G$ is similar to what we have described for $G L(N)$ above. It is a triplet $\left(G^{\prime}, s^{\prime}, \xi^{\prime}\right)$, where $G^{\prime}$ is a (connected) quasisplit group over $F, s^{\prime}$ is a semisimple element in $\widehat{G}$ of which $\widehat{G}^{\prime}$ is the connected centralizer, and $\xi^{\prime}$ is an $L$-embedding of ${ }^{L} G^{\prime}$ into ${ }^{L} G$. We again require that $\xi^{\prime}$ equal the identity on $\widehat{G}^{\prime}$, and that its image lie in the centralizer of $s^{\prime}$ in ${ }^{L} G$. (See [LS, (1.2)], a special case of the general definition in $[\mathbf{K S}]$, which we have specialized further to the case at hand.) There is again the notion of isomorphism of endoscopic data, which allows us to form the associated finite group

$$
\operatorname{Out}_{G}\left(G^{\prime}\right)=\operatorname{Aut}_{G}\left(G^{\prime}\right) / \operatorname{Int}_{G}\left(G^{\prime}\right)
$$

of any given $G^{\prime}$. (We again often let the endoscopic group $G^{\prime}$ stand for an endoscopic datum $\left(G^{\prime}, s^{\prime}, \xi^{\prime}\right)$, or an isomorphism class of data.) We write $\mathcal{E}(G)$ for the set of isomorphism classes of endoscopic data $G^{\prime}$ for $G$, and $\mathcal{E}_{\text {ell }}(G)$ for the subset of data that are elliptic, in the sense that $Z\left(\widehat{G}^{\prime}\right)^{\Gamma_{F}}$ is finite. We write $\mathcal{E}_{\text {sim }}(G)$ for the subset of data $G^{\prime} \in \mathcal{E}_{\text {ell }}(G)$ such that $Z\left(\widehat{G}^{\prime}\right)^{\Gamma_{F}}$ equals the minimal group $Z(\widehat{G})^{\Gamma_{F}}$. It consists of the simple group $G$ alone. We then have a second chain of sets

$$
\mathcal{E}_{\text {sim }}(G) \subset \mathcal{E}_{\text {ell }}(G) \subset \mathcal{E}(G),
$$

which is parallel to (3.4). Similar definitions apply to groups $G$ that represent more general data in $\widetilde{\mathcal{E}}(N)$.

The explicit description of elements $G^{\prime} \in \mathcal{E}_{\text {ell }}(G)$ is similar to our analysis of the set $\widetilde{\mathcal{E}}_{\text {ell }}(N)$ above. If $G \in \widetilde{\mathcal{E}}_{\text {sim }}(N)$ is of type $\mathbf{B}_{n}$, we have

$$
\widehat{G}^{\prime}=S p\left(N_{1}^{\prime}, \mathbb{C}\right) \times S p\left(N_{2}^{\prime}, \mathbb{C}\right) \subset S p(N, \mathbb{C})=\widehat{G},
$$

for a decomposition $N=N_{1}^{\prime}+N_{2}^{\prime}$ of $N=2 n$ into even integers with $0 \leq N_{1}^{\prime} \leq N_{2}^{\prime}$. If $G$ is of type $\mathbf{C}_{n}$, we have

$$
\widehat{G}^{\prime}=S O\left(N_{1}^{\prime}, \mathbb{C}\right) \times S O\left(N_{2}^{\prime}+1, \mathbb{C}\right) \subset S O(N, \mathbb{C})=\widehat{G},
$$


for a decomposition $N-1=N_{1}^{\prime}+N_{2}^{\prime}$ of $N-1=2 n$ into nonnegative, even integers. If $G$ is of type $\mathbf{D}_{n}$, we have

$$
\widehat{G}^{\prime}=S O\left(N_{1}^{\prime}, \mathbb{C}\right) \times S O\left(N_{2}^{\prime}, \mathbb{C}\right) \subset S O(N, \mathbb{C})=\widehat{G},
$$

for a decomposition $N=N_{1}^{\prime}+N_{2}^{\prime}$ of $N=2 n$ into even integers with $0 \leq N_{1}^{\prime} \leq N_{2}^{\prime}$. It is clear that the products are all connected centralizers of diagonal matrices $s^{\prime} \in \widehat{G}^{\prime}$ with entries \pm 1 . The reader will also be able to construct all endoscopic groups $G^{\prime}=G_{1}^{\prime} \times G_{2}^{\prime}$ with these dual groups, referring as needed to the end of $\S 1$, from quasisplit twists of the even orthogonal factors $G_{i}^{\prime}$. The endoscopic groups $G^{\prime}$ determine the endoscopic data $\left(G^{\prime}, s^{\prime}, \xi^{\prime}\right)$, unlike what was the case for twisted endoscopic data for $G L(N)$. (See the end of [ECR, $\S 1.3]$ for more remarks.)

We have completed our brief examination of elliptic, self-dual representations $r$. We are regarding these objects as parameters for $G L(N)$, in the spirit of $\S 2$. We have seen that any such parameter factors into a product of two parameters for two different quasisplit classical groups. These products are governed by twisted endoscopic data $G \in \widetilde{\mathcal{E}}_{\text {ell }}(N)$. They can be refined further according to ordinary endoscopic data $G^{\prime} \in \widetilde{\mathcal{E}}_{\text {ell }}(G)$. Thus, while the parameters will not be available in the global case of ultimate concern (for lack of a global Langlands group $L_{F}$ ), the endoscopic data that control many of their properties will be. Before we can examine the ramifications of this, however, we have first to formulate a makeshift substitute for global parameters attached to our quasisplit special orthogonal and symplectic groups. We shall do so in $\S 5$, after a discussion in $\S 4$ of the automorphic representations of $G L(N)$ that will serve as the global parameters for this group.

We have considered only the self-dual representations $r$ that are elliptic, since it is these objects that pertain directly to our global theorems. We might ask what happens if $r$ is not elliptic. With a little reflection, one sees that any such $r$ factors through subgroups of $G L(N, \mathbb{C})$ attached to several data $G \in \widetilde{\mathcal{E}}_{\text {ell }}(N)$, in contrast to what we have seen in the elliptic case. It also factors through subgroups attached to data $G \in \widetilde{\mathcal{E}}(N)$ in the complement of $\widetilde{\mathcal{E}}_{\text {ell }}(N)$. These matters are best formulated in terms of the centralizers

and

$$
\widetilde{S}_{r}(N)=S_{r}(\widetilde{G}(N))=\operatorname{Cent}(\operatorname{im}(r), \widehat{\widetilde{G}}(N))
$$

$$
S_{r}=S_{r}(G)=\operatorname{Cent}(\operatorname{im}(r), \widehat{G})
$$

of the images of $r$. Their analysis is closely tied to an elementary but important bijective correspondence (5.11) we will describe later.

\section{The case of $G L(N)$}

In $\S 2$, we introduced the kind of parameters that ought to characterize representations of a given group $G$. In $\S 3$, we examined how the parameters for an orthogonal or symplectic group $G$ are related to those for a general linear group $G L(N)$. This was in support of our goal, to classify representations of $G$ in terms of those of $G L(N)$. What makes the goal worthwhile is the fact that much of the representation theory of $G L(N)$ is well understood and relatively simple. In this section, we shall review what we need of the theory, following $[\mathbf{E C R}, \S 1.3]$ and $[\mathbf{A 5}$, $\S 1]$.

Assume for the time being that $F$ is global. If the global Langlands group $L_{F}$ existed, its corresponding set $\Psi(G L(N))$ of global parameters would be identified 
with the set of equivalence classes of unitary, $N$-dimensional representations of the locally compact group $L_{F} \times S U(2)$. Following notation from [ECR], we could then write $\Psi_{\operatorname{sim}}(G L(N))$ for the associated subset of irreducible representations. It would correspond to the set of pairs $(\mu, \nu)$, where $\mu$ is an irreducible unitary representation of $L_{F}$ of dimension $m$, and $\nu$ is the irreducible representation of $S U(2)$ of dimension $n$, for some decomposition $N=m n$ into a product of positive integers.

We do not, of course, have the group $L_{F}$ at our disposal. However, its irreducible $N$-dimensional representations are supposed to correspond to something we do have, cuspidal automorphic representations of $G L(N)$. We shall therefore take $\Psi_{\text {sim }}(N)=$ $\Psi_{\text {sim }}(G L(N))$ to be the set of pairs $(\mu, \nu)$ as above, with $\mu$ now being a unitary, cuspidal automorphic representation of $G L(m)$ instead of an irreducible, unitary $m$-dimensional representation of the hypothetical group $L_{F}$.

Theorem 4.1 (Moeglin-Waldspurger [MW1]). There is a canonical bijection

$$
\psi \longrightarrow \pi_{\psi}, \quad \psi \in \Psi_{\text {sim }}(N)
$$

from $\Psi_{\operatorname{sim}}(N)$ onto the set of irreducible unitary representations of $G L(N, \mathbb{A})$ that occur in the automorphic, relative discrete spectrum $L_{\text {disc }}^{2}(G L(N, F) \backslash G L(N, \mathbb{A}))$ of $G L(N)$. Moreover, for any $\psi, \pi_{\psi}$ occurs in the relative discrete spectrum with multiplicity one.

More generally, we let $\Psi(N)=\Psi(G L(N))$ denote the set of formal, unordered sums

$$
\psi=\psi_{1} \boxplus \cdots \boxplus \psi_{r}, \quad \psi_{i} \in \Psi_{\operatorname{sim}}\left(N_{i}\right),
$$

for some partition

$$
N=N_{1}+\cdots+N_{r}
$$

of $N$. It would correspond to the set

$$
\psi_{1} \oplus \cdots \oplus \psi_{r}
$$

of $N$-dimensional direct sums of irreducible unitary representations $\psi_{i}$ of the hypothetical group $L_{F} \times S U(2)$, or in other words, the set of all unitary, $N$-dimensional representations of $L_{F} \times S U(2)$.

COROLlary 4.2. There is a canonical bijection

$$
\psi \longrightarrow \pi_{\psi}, \quad \psi \in \Psi(N),
$$

from $\Psi(N)$ onto the set of irreducible constituents of the full automorphic spectrum $L^{2}(G L(N, F) \backslash G L(N, \mathbb{A}))$ of $G L(N)$.

The irreducible constituents of the automorphic spectrum are just the automorphic representations, according to the informal definition from $\S 1$ we are working with. The corollary tells us that there is a natural bijection from $\Psi(N)$ onto the set of automorphic representations of $G L(N)$. This is a consequence of Langlands' theory of Eisenstein series [L2], which provides an explicit construction of the automorphic continuous spectrum of any group in terms of (relative) discrete spectra for smaller groups.

If $\psi=(\mu, \nu)$ belongs to $\Psi_{\text {sim }}(N)$, Moeglin and Waldspurger construct the representation $\pi_{\psi}$ as a global Langlands quotient. It is by definition the unique irreducible quotient of the representation of $G L(n, \mathbb{A})$ obtained by parabolic induction 
from the nonunitary representation

$$
x \longrightarrow \mu\left(x_{1}\right)\left|\operatorname{det} x_{1}\right|^{\frac{n-1}{2}} \otimes \mu\left(x_{2}\right)\left|\operatorname{det} x_{2}\right|^{\frac{n-3}{2}} \otimes \cdots \otimes \mu\left(x_{n}\right)\left|\operatorname{det} x_{n}\right|^{-\frac{(n-1)}{2}}
$$

of the standard Levi subgroup

$$
M_{P}(\mathbb{A})=\left\{x=\left(x_{1}, \ldots, x_{n}\right): x_{i} \in G L(m, \mathbb{A})\right\} .
$$

More generally, if $\psi$ is a general element in $\Psi(N)$ of the form above, $\pi_{\psi}$ is the irreducible representation of $G L(N, \mathbb{A})$ obtained by parabolic induction from the unitary representation

$$
\pi_{\psi_{1}}\left(x_{1}\right) \otimes \cdots \otimes \pi_{\psi_{r}}\left(x_{r}\right)
$$

of the standard Levi subgroup

$$
M_{P}(\mathbb{A})=\left\{x=\left(x_{1}, \ldots, x_{r}\right): x_{i} \in G L\left(N_{i}, \mathbb{A}\right)\right\} .
$$

In both cases, Eisenstein series provide functions that represent intertwining operators from the representations $\pi_{\psi}$ to the associated constituents of the automorphic spectrum.

Suppose that $\pi$ is an irreducible representation of $G L(N, \mathbb{A})$. We assume implicitly that $\pi$ is admissible [F, p. 182], a broad condition that is always valid if $\pi$ is automorphic. The condition includes a property of weak continuity, which in view of the direct limit topology on the restricted direct product (1.1), implies that $\pi$ is unramified for almost all valuations $v$ of $F$. We alluded to unramified representations in our heuristic description of the general global packets (2.2) and (2.7). For the group $G=G L(N)$, an irreducible representation $\pi_{v}$ of $G\left(F_{v}\right)$ is unramified by definition if $F_{v}$ is nonarchimedean, and the restriction of $\pi_{v}$ to the open compact subgroup $G\left(\mathfrak{o}_{v}\right)$ of $G\left(F_{v}\right)$ contains the trivial, 1-dimensional representation. Unramified representations have a very simple classification. A well known integral transform, introduced into $p$-adic harmonic analysis by Satake, leads to a canonical bijection

$$
\pi_{v} \longrightarrow c\left(\pi_{v}\right)
$$

from the set of irreducible unramified representations $\pi_{v}$ of $G L\left(N, F_{v}\right)$ onto the set of semisimple conjugacy classes $c_{v}$ in the dual group $G L(N, \mathbb{C})$ of $G L(N)$. The given global representation $\pi$ thus gives rise to a family of conjugacy classes

$$
c^{S}(\pi)=\left\{c_{v}(\pi)=c\left(\pi_{v}\right): v \notin S\right\}
$$

parametrized by a cofinite set of valuations of $F$. In order to remove its dependence on $S$, some finite set that contains the archimedean valuations, we write $c(\pi)$ for the equivalence class of $c^{S}(\pi)$ with respect to the relation defined by setting $c^{S} \sim\left(c^{\prime}\right)^{S^{\prime}}$ if $c_{v}=c_{v}^{\prime}$ for almost all $v$.

We call $c(\pi)$ (or any of its representatives $c^{S}(\pi)$ within the equivalence class) a Hecke eigenfamily. It represents a set of simultaneous eigenvalues for the action of the factors of the restricted tensor product

$$
\mathcal{H}_{\mathrm{un}}^{S}(N)=\bigotimes_{v \notin S}^{\sim} \mathcal{H}_{v, \mathrm{un}}(N)
$$

of local unramified Hecke algebras

$$
\mathcal{H}_{v, \text { un }}(N)=C_{c}^{\infty}\left(G L\left(N, \mathfrak{o}_{v}\right) \backslash G L\left(N, F_{v}\right) / G L\left(N, \mathfrak{o}_{v}\right)\right)
$$


under convolution, relative to the hyperspecial maximal compact subgroup

$$
G L\left(N, \mathfrak{o}^{S}\right)=\prod_{v \notin S} G L\left(N, \mathfrak{o}_{v}\right)
$$

of $G L\left(N, \mathbb{A}^{S}\right)$, on the space of $G L\left(N, \mathfrak{o}^{S}\right)$-invariant vectors of $\pi^{S}$. Our interest of course is the case that $\pi$ is automorphic.

Suppose that $\psi$ is an element (4.1) in the set $\Psi(N)$. We then obtain a Hecke eigenfamily

$$
c^{S}(\psi)=c^{S}\left(\pi_{\psi}\right)=\left\{c_{v}(\psi)=c\left(\pi_{\psi, v}\right): v \notin S\right\},
$$

with equivalence class $c(\psi)=c\left(\pi_{\psi}\right)$, from the irreducible representation $\pi_{\psi}$ of $G L(N, \mathbb{A})$. It is to be regarded as a concrete datum, which is attached to the formal object $\psi$ through the automorphic representation $\pi_{\psi}$. According to the remarks following the statement of Corollary $4.2, c\left(\psi_{i}\right)$ will be given explicitly in terms of Hecke eigenfamilies $c\left(\mu_{i}\right)$, represented by sets

$$
c^{S}\left(\mu_{i}\right)=\left\{c_{v}\left(\mu_{i}\right): v \notin S\right\}, \quad 1 \leq i \leq r,
$$

which we extract from the cuspidal components $\mu_{i}$ of constituents $\psi_{i}$ of $\psi$. More precisely, if $\psi=(\mu, \nu)$ belongs to $\Psi_{\text {sim }}(N)$ then

$$
c_{v}(\psi)=c_{v}(\mu) \otimes c_{v}(\nu)=c_{v}(\mu) q_{v}^{\frac{n-1}{2}} \oplus \cdots \oplus c_{v}(\mu) q_{v}^{-\frac{n-1}{2}}, \quad v \notin S,
$$

while if $\psi$ is a general element (4.1) in $\Psi(N)$, we have

$$
c_{v}(\psi)=c_{v}\left(\psi_{1}\right) \oplus \cdots \oplus c_{v}\left(\psi_{r}\right), \quad v \notin S .
$$

These elements are to be regarded as diagonal matrices in $G L(N, \mathbb{C})$, which of course represent semisimple conjugacy classes.

We write

$$
\mathcal{C}(N)=\{c(\psi): \psi \in \Psi(N)\}
$$

for the set of Hecke eigenfamilies attached to elements in $\Psi(N)$.

TheOREM 4.3 (Jacquet-Shalika [JS]). The mapping

$$
\psi \longrightarrow c(\psi), \quad \psi \in \Psi(N),
$$

is a bijection from $\Psi(N)$ to $\mathcal{C}(N)$.

As we noted in $[\mathbf{A 5}, \S 1]$, which we have been following closely here, Theorem 4.3 predates Theorem 1.1. As stated in [JS $]$, it applied to a class of automorphic representations Langlands introduced in $[\mathbf{L} 4]$, and called isobaric. At the time, it was not known whether this class included the constituents of the automorphic (relative) discrete spectrum. Theorem 4.1 implies that such constituents are distinct and isobaric. It therefore yields the interpretation we have stated above for the original theorem of Jacquet and Shalika.

Theorem 4.3 is a striking result. It implies that any information that might be contained in a constituent $\pi_{\psi}$ of the automorphic spectrum of $G L(N)$ will be captured in the corresponding Hecke eigenfamily $c(\psi)$. Since $c(\psi)$ appears to contain less information, and since it is just a concrete set of complex parameters, the assertion is indeed remarkable. It is the more so for our expressed goal of classifying automorphic representations of the other groups $G$ in terms of those of $G L(N)$, and thus in terms of Hecke eigenfamilies $c(\psi)$. 
Before turning to the other groups, we shall discuss the local theory for $G L(N)$. The local Langlands correspondence is known in this case. In order to review it, we shall first say something about local $L$-functions and $\varepsilon$-factors.

Assume now that $F$ is local. There are two kinds of local $L$-functions, arithmetic and representation theoretic. The former are attached to finite dimensional representations of the local Langlands group $L_{F}$, the latter to irreducible representations of general linear groups over $F$.

Suppose that

$$
\phi: L_{F} \longrightarrow G L(N, \mathbb{C})
$$

is an $N$-dimensional (semisimple, continuous) representation of $L_{F}$. The associated arithmetic $L$-function $L(s, \phi)$ is a meromorphic function of $s \in \mathbb{C}$. One can also form the local arithmetic $\varepsilon$-factor $\varepsilon\left(s, \phi, \psi_{F}\right)$, a monomial of the form $a b^{-s}$, which depends on a nontrivial additive character $\psi_{F}$ of $F$. The definition of the coefficient $a$ for nonarchimedean $F$ is by far the most subtle part of the process. It was constructed canonically by Deligne, following ideas of Artin and Langlands, by a global argument. If $F$ is archimedean, we refer the reader to the definition in [ $\mathbf{T}, \S 3]$. If $F$ is nonarchimedean, one extends $\phi$ analytically to a representation to the product of $W_{F}$ with the complexification $S L(2, \mathbb{C})$ of the compact unitary subgroup $S U(2)$ of $L_{F}$. This gives a representation

$$
\mu_{\phi}(w)=\phi\left(w,\left(\begin{array}{cc}
|w|^{\frac{1}{2}} & 0 \\
0 & |w|^{-\frac{1}{2}}
\end{array}\right)\right), \quad w \in W_{F},
$$

of $W_{F}$, in which $|w|$ is the absolute value on $W_{F}$, and a nilpotent matrix

$$
N_{\phi}=\log \left(\phi\left(1,\left(\begin{array}{ll}
1 & 1 \\
0 & 1
\end{array}\right)\right)\right) .
$$

The pair $V_{\phi}=\left(\mu_{\phi}, N_{\phi}\right)$ provides representation of what is known as the WeilDeligne group, from which one can define an $L$-function

$$
L(s, \phi)=Z\left(V_{\phi}, q_{F}^{-s}\right)
$$

and $\varepsilon$-factor

$$
\varepsilon\left(s, \phi, \psi_{F}\right)=\varepsilon\left(V_{\phi}, q_{F}^{-s}\right),
$$

with $q_{F}$ being the order of the residue field of $F$, following notation in [T, $\left.\S 4\right]$. Of particular interest are the tensor product $L$-function

$$
L\left(s, \phi_{1} \times \phi_{2}\right)=L\left(s, \phi_{1} \otimes \phi_{2}\right)
$$

and $\varepsilon$-factor

$$
\varepsilon\left(s, \phi_{1} \times \phi_{2}, \psi_{F}\right)=\varepsilon\left(s, \phi_{1} \otimes \phi_{2}, \psi_{F}\right)
$$

attached to any pair of representations $\phi_{1}$ and $\phi_{2}$ of $L_{F}$.

Representation theoretic $L$-functions $L(s, \pi, r)$ and $\varepsilon$-factors $\varepsilon\left(s, \pi, r, \psi_{F}\right)$ ought to be attached naturally to irreducible representations $\pi$ of $G(F)$ and finite dimensional representations $r$ of ${ }^{L} G$, for any reductive group $G$ over $F$. For general $G$, this can be done in only relatively simple cases. However, if $G$ is a product $G L\left(N_{1}\right) \times G L\left(N_{2}\right)$ of general linear groups, there is a broader theory [JPS]. It applies to any representation $\pi=\pi_{1} \otimes \pi_{2}$, with $r$ being the standard representation

$$
r\left(g_{1}, g_{2}\right): X \longrightarrow g_{1} X^{t} g_{2}, \quad g \in G L\left(N_{i}, \mathbb{C}\right),
$$

of

$$
\widehat{G}=G L\left(N_{1}, \mathbb{C}\right) \times G L\left(N_{2}, \mathbb{C}\right)
$$


on the space of complex $\left(N_{1} \times N_{2}\right)$-matrices $X$. The theory yields functions

$$
L\left(s, \pi_{1} \times \pi_{2}\right)=L(s, \pi, r)
$$

and

$$
\varepsilon\left(s, \pi_{1} \times \pi_{2}, \psi_{F}\right)=\varepsilon\left(s, \pi, r, \psi_{F}\right)
$$

known as Rankin-Selberg products.

We write $\Phi(N)=\Phi(G L(N)), \Phi_{\text {bdd }}(N)=\Phi_{\text {bdd }}(G L(N))$ and $\Psi(N)=$ $\Psi(G L(N))$ for the specialization to $G L(N)$ of the local parameter sets from $\S 2$, and $\Pi(N)=\Pi(G L(N)), \Pi_{\text {temp }}(N)=\Pi_{\text {temp }}(G L(N))$ and $\Pi_{\text {unit }}(N)=\Pi_{\text {unit }}(G L(N))$ for the associated sets of irreducible representations of $G L(N, F)$. The local Langlands correspondence applies to the first of these. It is characterized essentially by its compatibility with Rankin-Selberg products.

Theorem 4.4 (Langlands [L5], Harris-Taylor [HT], Henniart [H], Scholze $[\mathbf{S c h}])$. There is a canonical bijective correspondence

$$
\phi \longrightarrow \pi_{\phi}
$$

from $\Phi(N)$ to $\Pi(N)$ such that

$$
L\left(s, \pi_{\phi_{1}} \times \pi_{\phi_{2}}\right)=L\left(s, \phi_{1} \times \phi_{2}\right)
$$

and

$$
\varepsilon\left(s, \pi_{\phi_{1}} \times \pi_{\phi_{2}}, \psi_{F}\right)=\varepsilon\left(s, \phi_{1} \times \phi_{2}, \psi_{F}\right),
$$

for any pair of local parameters $\phi_{1} \in \Phi\left(N_{1}\right)$ and $\phi_{2} \in \Phi\left(N_{2}\right)$.

The correspondence also satisfies other natural conditions. These include compatibility with the automorphism $\tilde{\theta}(N)$ of $G L(N)$, with tensor products by 1dimensional representations, and with the one-dimensional correspondence given by class field theory (as it relates to determinants and central characters). If we append these three supplementary conditions to its compatibility (4.8) and (4.9) with Rankin-Selberg products, the correspondence becomes unique. It is in this sense that it is canonical.

The local correspondence has other properties as well. It restricts to a bijection from the subset $\Phi_{\text {bdd }}(N)$ of $\Phi(N)$ to the subset $\Pi_{\text {temp }}(N)$ of $\Pi(N)$. It restricts further to a bijection from the subset $\Phi_{\text {sim,bdd }}(N)$ of irreducible representations in $\Phi_{\text {bdd }}(N)$ to the subset $\Pi_{2, \text { temp }}(N)$ of irreducible representations of $G L(N, F)$ that occur in the relative discrete spectrum of $L^{2}(G L(N, F))$. In the case that $F$ is nonarchimedean, it also takes the subset of representations $\phi$ in $\Phi_{\text {sim,temp }}(N)$ that are trivial on the factor $S U(2)$ of $L_{F}$ onto the subset of representations in $\Pi_{\text {sim,temp }}(N)$ that are supercuspidal.

The local Langlands correspondence also bears on the local set $\Psi(N)$. For any $\psi \in \Psi(N)$, we write

$$
\phi_{\psi}(w)=\psi\left(w,\left(\begin{array}{cc}
|w|^{\frac{1}{2}} & 0 \\
0 & |w|^{-\frac{1}{2}}
\end{array}\right)\right), \quad w \in L_{F}
$$

where $|w|$ is now the pullback to $L_{F}$ of the absolute value on $W_{F}$. We obtain a mapping

$$
\psi \longrightarrow \phi_{\psi}, \quad \psi \in \Psi(N)
$$


from $\Psi(N)$ to $\Phi(N)$, which is in fact injective, as one sees easily from the fact that restriction of $\psi$ to $L_{F}$ lies in $\Phi_{\text {bdd }}(N)$. The representation

$$
\pi_{\psi}=\pi_{\phi_{\psi}}, \quad \psi \in \Psi(N)
$$

defined by the local correspondence is called a Speh representation. It is known to be unitary. We thus have an injective mapping

$$
\psi \longrightarrow \pi_{\psi}
$$

from $\Psi(N)$ to $\Pi_{\text {unit }}(N)$. This is the local analogue of the global bijection of Corollary 4.1.2. In contrast to the global setting, however, it does not have a natural interpretation in terms of local harmonic analysis. It is also not surjective.

Suppose that $\psi$ is a general local parameter in $\Psi(N)$. The centralizer $S_{\psi}$ defined (2.8) in $\S 2$ is the group of invertible intertwining operators of the $N$-dimensional representation given by $\psi$. It is a product of complex general linear groups, embedded diagonally in $G L(N, \mathbb{C})$, and is therefore connected. The associated group of connected components $\mathcal{S}_{\psi}$ is consequently trivial. Our heuristic discussion at the end of $\S 2$ suggests that the packet $\Pi_{\psi}$ ought then to consist of only the one representation $\pi_{\psi}$. This in fact matches the formal definition we will discuss in $\S 6$. It is one more indication that the representation theory of $G L(N)$ is simpler than that of other groups.

Suppose again that $F$ is global. If $\psi=(\mu, \nu)$ belongs to the global set $\Psi_{\operatorname{sim}}(N)$, any local constituent $\pi_{\psi, v}$ of the automorphic representation of Theorem 4.1 equals the Speh representation $\pi_{\psi_{v}}$ attached to the local parameter ${ }^{7} \psi_{v}=\mu_{v} \otimes \nu$. This is not hard to establish from the definitions of $\pi_{\psi}$ and $\pi_{\psi_{v}}$ as Langlands quotients. The generalized Ramanujan conjecture asserts that as a local constituent of $\mu$, $\mu_{v}$ is a tempered representation of $G L\left(m, F_{v}\right)$, or equivalently, that it is a unitary $m$-dimensional representation of $L_{F_{v}}$. Since we do not know that this conjecture is valid, however, we can assume only that the local parameter $\psi_{v}$ lies in the set $\Psi_{v}^{+}(N)$ of $N$-dimensional, not necessarily unitary representations of $L_{F_{v}} \times S U(2)$. More generally, suppose that

$$
\psi=\psi_{1} \boxplus \cdots \boxplus \psi_{r}, \quad \psi_{k} \in \Psi_{\operatorname{sim}}\left(N_{k}\right),
$$

is a general element in $\Psi(N)$. As in the case above that $\psi$ is simple, one sees that

$$
\pi_{\psi, v}=\pi_{\psi_{v}},
$$

for the local parameter

$$
\psi_{v}=\psi_{1, v} \oplus \cdots \oplus \psi_{r, v}
$$

in $\Psi_{v}^{+}(N)$. We thus obtain a natural localization mapping

$$
\psi \longrightarrow \psi_{v}, \quad \psi \in \Psi(N)
$$

from $\Psi(N)$ to $\Psi_{v}^{+}(N)$. We also see that the global packet $\Pi_{\psi}$, defined heuristically (2.7) in terms of $L_{F}$, should consist of the one representation

$$
\pi_{\psi}=\bigotimes_{v} \pi_{\psi_{v}}
$$

\footnotetext{
${ }^{7}$ We are writing $\mu_{v}$ for both the local constituent of the cuspidal automorphic representation $\mu$ of $G L(m)$, and the $m$-dimensional representation of $L_{F_{v}}$ to which it corresponds. This is natural if we consider that $\mu$ should really represent an irreducible, $m$-dimensional representation of the global group $L_{F}$.
} 
Once again, the representation theory of $G L(N)$ seems to be about as simple as it can be.

As we noted in $\S 2$, global packets should have implications for global $L$-functions. Arithmetic global $L$-functions and $\varepsilon$-factors are attached to $N$-dimensional representations $\phi$ of $W_{F}$ (or more generally, of the hypothetical group $L_{F}$ ). They are defined as products

$$
L(s, \phi)=\prod_{v} L\left(s, \phi_{v}\right)
$$

and

$$
\varepsilon(s, \phi)=\prod_{v} \varepsilon\left(s, \phi_{v}, \psi_{F_{v}}\right)
$$

of their local analogues, where $\psi_{F}$ is a nontrivial additive character on the quotient $\mathbb{A} / F$. Automorphic (representation theoretic) versions are attached to admissible representations $\pi$ of $G(\mathbb{A})$ and finite dimensional representations $r$ of ${ }^{L} G$, for any reductive group $G$ over $F$. They are again defined as products

$$
L(s, \pi, r)=\prod_{v} L\left(s, \pi_{v}, r_{v}\right)
$$

and

$$
\varepsilon(s, \pi, r)=\prod_{v} \varepsilon\left(s, \pi_{v}, r_{v}, \psi_{F_{v}}\right)
$$

of their localizations. The products of $\varepsilon$-factors can be taken over a finite set. The products of $L$-functions converge for the real part of $s$ in some right half plane. ${ }^{8}$ They should have analytic continuation ${ }^{9}$ to meromorphic functions of $s$, which satisfy the functional equations

$$
L(s, \phi)=\varepsilon(s, \phi) L\left(1-s, \phi^{\vee}\right)
$$

and

$$
L(s, \pi, r)=\varepsilon(s, \pi, r) L\left(1-s, \pi, r^{\vee}\right),
$$

where $\phi^{\vee}$ and $r^{\vee}$ denote the contragredients of the finite dimensional representations $\phi$ and $r$. The two kinds of objects should ultimately be related by identities

$$
L(s, \pi, r)=L(s, r \circ \phi)
$$

and

$$
\varepsilon(s, \pi, r)=\varepsilon(s, r \circ \phi),
$$

for any representation $\pi$ in the global packet of $\phi$. However, these "reciprocity laws" are still far away in general, and as in the local case, one needs to study the arithmetic and automorphic objects independently.

The correspondence $\psi \rightarrow \phi_{\psi}$ of (4.10) will be completely general. As in the local case discussed for $G L(N)$ above, it will map general parameters $\psi$ on $L_{F} \times S U(2)$ injectively to parameters on the group $L_{F}$. One then defines arithmetic $L$-functions and $\varepsilon$-factors for any $\psi$ and $r$ by

$$
L(s, r \circ \psi)=L\left(s, r \circ \phi_{\psi}\right)
$$

\footnotetext{
8 In the representation theoretic case, one must impose a property of weak growth on $\pi$, which always holds if $\pi$ is automorphic.

${ }^{9}$ In the representation theoretic case, $\pi$ should now be automorphic.
} 
and

$$
\varepsilon(s, r \circ \psi)=L\left(s, r \circ \phi_{\psi}\right) .
$$

They should be equal to representation theoretic functions $L\left(s, \pi_{\psi}, r\right)$ and $\varepsilon\left(s, \pi_{\psi}, r\right)$ respectively, for any representation $\pi_{\psi}$ in the global packet $\Pi_{\phi_{\psi}}$.

A reader unfamiliar with these notions might be overwhelmed now with the details of what has taken the world of number theory many years to absorb, while at the same time, an expert might be quite tired of my repetition. At the risk of exhausting the patience of both parties, let me say a word on the concrete roots of global $L$-functions.

An irreducible admissible representation $\pi$ of $G(\mathbb{A})$ still has unramified local constituents $\pi_{v}$ at almost all $v$. Moreover, the Satake transform (4.2) remains valid. It is a bijection from the set of unramified representations $\pi_{v}$ of $G\left(F_{v}\right)$ onto the set of semisimple conjugacy classes $c\left(\pi_{v}\right)$ in the dual group whose image in the factor $W_{F_{v}}$ of ${ }^{L} G_{v}$ is a Frobenius element. The given global representation $\pi$ then gives rise to a family

$$
c^{S}(\pi)=\left\{c_{v}(\pi)=c\left(\pi_{v}\right): v \notin S\right\}
$$

of semisimple conjugacy classes in ${ }^{L} G$, and a corresponding equivalence class $c(\pi)$ that is independent of $S$. For any unramified representation $r_{v}$ of ${ }^{L} G_{v}$, the local $L$-function of $\pi_{v}$ and $r_{v}$ is defined

$$
L\left(s, \pi_{v}, r_{v}\right)=\operatorname{det}\left(1-r_{v}\left(c\left(\pi_{v}\right)\right) q_{v}^{-s}\right)^{-1}, \quad q_{v}=q_{F_{v}},
$$

in terms of the characteristic polynomial of the conjugacy class $r_{v}\left(c\left(\pi_{v}\right)\right)$. If $r$ is allowed to range over representations of ${ }^{L} G$ that are unramified outside $S$ (a set we can allow to vary), the partial global $L$-functions

$$
L^{S}(s, \pi, r)=\prod_{v \notin S} L\left(s, \pi_{v}, r_{v}\right)
$$

give an elegant way to package the concrete data from $c(\pi)$. If $\pi$ is automorphic, each associated partial $L$-function is supposed to have analytic continuation to a meromorphic function of $s$, which satisfies a functional equation that is now quite complex. The object is then to define supplementary factors $L\left(s, \pi_{v}, r_{v}\right)$ (which should be relatively straightforward) for $v \in S$, and $\varepsilon\left(s, \pi_{v}, r_{v}, \psi_{v}\right)$ (which are deep if $v \in S$, but equal to 1 if $v \notin S$ ) so that the products (4.13) and (4.14) satisfy the simple functional equation (4.15). The Langlands-Shahidi method [Sha] has been the most successful technique for studying these questions, but as in the local situation, it applies only to relatively simple cases.

Global Rankin-Selberg products are well understood, thanks again to [JPS]. They apply to the representation $r$ of the group $G=G L\left(N_{1}\right) \times G L\left(N_{2}\right)$ on the space of $\left(N_{1} \times N_{2}\right)$-matrices. Suppose that $\psi_{1} \in \Psi\left(N_{1}\right)$ and $\psi_{2} \in \Psi\left(N_{2}\right)$ are elements in the sets we have introduced as substitutes for general global parameters. The corresponding representations

$$
\pi_{i}=\pi_{\psi_{i}}=\pi_{\phi_{\psi_{i}}}, \quad i=1,2,
$$

of $G L\left(N_{i}, \mathbb{A}\right)$ are automorphic, according to Corollary 4.2. We write

$$
L\left(s, \psi_{1} \times \psi_{2}\right)=L\left(s, \pi_{1} \times \pi_{2}\right)
$$

and

$$
\varepsilon\left(s, \psi_{1} \times \psi_{2}\right)=\varepsilon\left(s, \pi_{1} \times \pi_{2}\right)
$$


for the associated global $L$-functions and $\varepsilon$-factors (4.13) and (4.14). It follows from [JPS] that they have analytic continuation and functional equation (4.15). The definitions (4.18) and (4.19) are suggestive. They look like examples of the fundamental reciprocity laws between arithmetic and automorphic $L$-functions. But because we have had to define the global sets $\Psi(N)$ in terms of cuspidal automorphic representations instead of representations of the Langlands group $L_{F}$, they remain just definitions. However, in a formal sense, they confirm the expectation that global $L$-functions attached to representations in global packets (2.2) and (2.7) should have analytic continuation and functional equation. They also represent formal global versions of the compatibility conditions (4.8) and (4.9) that characterize the local Langlands correspondence.

\section{Global parameters for $G$}

In the last section we introduced a set of global "parameters" $\Psi(N)$ for $G L(N)$. It is our substitute for the actual set of parameters, which would consist of unitary representations of the hypothetical global group $L_{F} \times S U(2)$. We also saw how to describe the automorphic representation theory of $G L(N)$ in terms of $\Psi(N)$. In this section, we turn to our classical groups $G$. Motivated by the heuristic discussion of $\S 3$, we will try to identify the subset $\Psi(G)$ of $\Psi(N)$ that will serve as a set of global parameters for $G$. Among other things, we will have to attach a subgroup $S_{\psi}$ of $\widehat{G}$ to any $\psi \in \Psi(G)$, since its group of connected components governs global multiplicities. We follow the discussion in [ECR, §1.4].

We assume in this section that the field $F$ is global. The notation (4.1) for a general element $\psi \in \Psi(N)$ should not be confused with the similar way we denoted an elliptic representation (3.2) of the abstract group $\Lambda_{F}$. This is because the simple factors $\psi_{i}$ in (4.1) are not required to be distinct. In future, we shall prefer the analogue of the notation (3.1). That is, we denote a general element in $\Psi(N)$ as a formal, unordered direct sum

$$
\psi=\ell_{1} \psi_{1} \boxplus \cdots \boxplus \ell_{r} \psi_{r},
$$

for positive integers $\ell_{k}$ and distinct elements $\psi_{k}=\left(\mu_{k}, \nu_{k}\right)$ in $\Psi_{\operatorname{sim}}\left(N_{k}\right)$. The ranks are positive integers $N_{k}=m_{k} n_{k}$ such that

$$
N=\ell_{1} N_{1}+\cdots+\ell_{r} N_{r}=\ell_{1} m_{1} n_{1}+\cdots+\ell_{r} m_{r} n_{r} .
$$

We will also often denote the simple components $\psi_{k}$ of $\psi$ as formal tensor products

$$
\psi_{k}=\left(\mu_{k}, \nu_{k}\right)=\mu_{k} \otimes \nu_{k},
$$

since they replace tensor products of irreducible representations of the two groups $L_{F}$ and $S U(2)$.

We are looking to $\S 3$ for guidance. The essential objects there were the representations of $\Lambda_{F}$, an abstract group over $W_{F}$, that were self-dual. The corresponding duality operator on $\Psi(N)$ is given by the outer automorphism $\theta: x \rightarrow x^{\vee}$ of $G L(N)$. It transforms a general parameter (5.1) to its contragredient

$$
\begin{aligned}
\psi^{\vee} & =\ell_{1} \psi_{1}^{\vee} \boxplus \cdots \boxplus \ell_{r} \psi_{r}^{\vee} \\
& =\ell_{1}\left(\mu_{1}^{\vee} \otimes \nu_{1}\right) \boxplus \cdots \boxplus \ell_{r}\left(\mu_{r}^{\vee} \otimes \nu_{r}\right),
\end{aligned}
$$

since

$$
\psi_{k}^{\vee}=\left(\mu_{k} \otimes \nu_{k}\right)^{\vee}=\mu_{k}^{\vee} \otimes \nu_{k}^{\vee}=\mu_{k}^{\vee} \otimes \nu_{k}
$$


We are writing

$$
\mu_{k}^{\vee}(x)=\mu_{k}\left({ }^{t} x^{-1}\right) \cong{ }^{t} \mu_{k}(x)^{-1}, \quad x \in G L\left(m_{k}, \mathbb{A}\right),
$$

for the contragredient of the cuspidal automorphic representation $\mu_{k}$ of $G L\left(m_{k}\right)$, and we have written $\nu_{k}^{\vee}=\nu_{k}$, since any representation of $S U(2)$ is self-dual. The contragredient $\pi_{\psi}^{\vee}$ of the associated automorphic representation $\pi_{\psi}$ of $G L(N)$ then equals $\pi_{\psi^{\vee}}$, as follows from the various definitions. Since the local correspondence of Theorem 4.4 commutes with duality, according to one of its supplementary conditions, the mapping $\psi \rightarrow \psi^{\vee}$ is the analogue for $\Psi(N)$ of duality for $N$-dimensional representations of $L_{F} \times S U(2)$.

We write

$$
\widetilde{G}(N)=\widetilde{G}(N)^{0} \rtimes \widetilde{\theta}(N)=G L(N) \rtimes \theta
$$

as in $\S 3$ for the nonidentity connected component of the semidirect product

$$
\widetilde{G}(N)^{+}=\widetilde{G}(N)^{0} \rtimes \widetilde{\theta}(N)^{+}=G L(N) \rtimes \theta^{+}
$$

of $G L(N)$ with the group of order 2 generated by either of the automorphisms $\widetilde{\theta}(N)$ or $\theta$. We also write

$$
\widetilde{\Psi}(N)=\Psi(\widetilde{G}(N))=\left\{\psi \in \Psi(N): \psi^{\vee}=\psi\right\}
$$

for the set of self-dual elements in $\Psi(N)$. This subset is indeed associated with the component $\widetilde{G}(N)$. It consists of the elements $\psi \in \Psi(N)$ such that the automorphic representation $\pi_{\psi} \cong \pi_{\psi}^{\vee}$ of $G L(N)=\widetilde{G}(N)^{0}$ has an extension to the group $\widetilde{G}(N, \mathbb{A})^{+}$ generated by $\widetilde{G}(N, \mathbb{A})$. We observe also that there is pointwise action

$$
c=\left\{c_{v}\right\} \longrightarrow c^{\vee}=\left\{c_{v}^{\vee}\right\}
$$

of $\theta$ on the set of (equivalence classes of) Hecke eigenfamilies $\mathcal{C}(N)$. The mapping of Theorem 4.3 restricts to a bijection from $\widetilde{\Psi}(N)$ onto the subset

$$
\widetilde{\mathcal{C}}(N)=\left\{c \in \mathcal{C}(N): c^{\vee}=c\right\}
$$

of self-dual elements in $\mathcal{C}(N)$.

We are particularly interested in the subset

$$
\widetilde{\Psi}_{\operatorname{sim}}(N)=\widetilde{\Psi}(N) \cap \Psi_{\operatorname{sim}}(N)
$$

of $\widetilde{\Psi}(N)$. It consists of the simple parameters $\psi=\mu \otimes \nu$ in $\widetilde{\Psi}(N)$. Among these, we have the smaller subset

$$
\widetilde{\Phi}_{\text {sim }}(N)=\widetilde{\Psi}_{\text {cusp }}(N)
$$

of parameters that are generic, in the sense that $\nu$ is trivial. A simple generic parameter is therefore a self-dual, cuspidal automorphic representation of $G L(N)$.

The term simple was also applied in $\S 1.2$ to endoscopic data. It was used to designate the subset $\widetilde{\mathcal{E}}_{\text {sim }}(N)$ of elliptic twisted endoscopic data $G \in \widetilde{\mathcal{E}}_{\text {ell }}(N)$ that are not composite. An element in $\widetilde{\mathcal{E}}_{\text {sim }}(N)$ is therefore one of our quasisplit, special orthogonal or symplectic groups $G$ over $F$, but armed with some extra structure (namely, a choice of endoscopic embedding) in case $G=S p(2 n)$ and $\widehat{G}=S O(2 n+$ $1, \mathbb{C})$. For any such $G$, we need to introduce the subset $\widetilde{\Psi}(G)$ of $\widetilde{\Psi}(N)$ that will serve as global parameters for $G$. Its construction will be based on the following fundamental case. 
THEOREM 5.1. Suppose that $\phi \in \widetilde{\Phi}_{\text {sim }}(N)$ is a simple generic global parameter. Then there is a unique $G_{\phi} \in \widetilde{\mathcal{E}}_{\mathrm{ell}}(N)$, regarded as an isomorphism class of twisted endoscopic data $\left(G_{\phi}, s_{\phi}, \xi_{\phi}\right)$ for $G L(N)$, such that

$$
c(\phi)=\xi_{\phi}(c(\pi))
$$

for some automorphic representation $\pi$ of $G$. Moreover, $G_{\phi}$ is simple, and $\pi$ occurs in the automorphic discrete spectrum $L_{\mathrm{disc}}^{2}(G(F) \backslash G(\mathbb{A}))$.

The theorem asserts that among all twisted elliptic endoscopic data $G \in \widetilde{\mathcal{E}}_{\text {ell }}(N)$, there is exactly one that contains the automorphic source for the Hecke eigenfamily $c(\phi)$ of $\phi$. The result serves as a "seed theorem" for our construction of $\widetilde{\Psi}(G)$. However, it has to be proved at the same time as broader theorems that pertain to the set $\widetilde{\Psi}(G)$ under construction. In this process, Theorem 5.1 becomes an induction hypothesis in the general treatment of parameters in $\widetilde{\Psi}(G)$. We shall assume it in what follows.

Suppose that $\psi$ is a fixed element in $\widetilde{\Psi}(N)$. It is convenient to write $K_{\psi}$ for the set $\{1, \ldots, r\}$ that indexes the simple constituents $\psi_{k}$ of $\psi$. Since $\psi$ is self-dual, there is an involution $k \rightarrow k^{\vee}$ on $K_{\psi}$ such that $\psi_{k^{\vee}}=\psi_{k}^{\vee}$ and $\ell_{k} \vee=\ell_{k}$. The indexing set is then a disjoint union

$$
K_{\psi}=I_{\psi} \amalg J_{\psi} \amalg J_{\psi}^{\vee}, \quad J_{\psi}^{\vee}=\left\{j^{\vee}: j \in J_{\psi}\right\},
$$

where $I_{\psi}$ is the set of fixed points of the involution, and $J_{\psi}$ is some complementary set of representatives of the orbits of order 2 . With this notation, we write

$$
\psi=\left(\underset{i \in I_{\psi}}{\boxplus} \ell_{i} \psi_{i}\right) \boxplus\left(\underset{j \in J_{\psi}}{\boxplus} \ell_{j}\left(\psi_{j} \boxplus \psi_{j} \vee\right)\right) .
$$

If $i$ belongs to $I_{\psi}$, we apply Theorem 5.1 to the simple generic factor $\mu_{i} \in \widetilde{\Phi}_{\text {sim }}\left(m_{i}\right)$ of $\psi_{i}=\mu_{i} \otimes \nu_{i}$. This gives an endoscopic datum $\left(G_{\mu_{i}}, s_{\mu_{i}}, \xi_{\mu_{i}}\right)$ in $\widetilde{\mathcal{E}}_{\text {sim }}\left(m_{i}\right)$, which we denote by $H_{i}$. If $j$ belongs to $J_{\psi}$, we set $H_{j}=G L\left(m_{j}\right)$. We thus obtain a connected, reductive group $H_{k}$ over $F$ for any index $k$ in either $I_{\psi}$ or $J_{\psi}$, which is to say, in the set

$$
\left\{K_{\psi}\right\} \cong I_{\psi} \amalg J_{\psi}
$$

of orbits of the involution on $K_{\psi}$. Let ${ }^{L} H_{k}$ be the Galois form of its $L$-group. We can then form the fibre product

$$
\mathcal{L}_{\psi}=\prod_{k \in\left\{K_{\psi}\right\}}\left({ }^{L} H_{k} \longrightarrow \Gamma_{F}\right)
$$

of these groups over $\Gamma_{F}$.

We will use the group $\mathcal{L}_{\psi}$ as a substitute for the global Langlands group $L_{F}$ in our study of automorphic representations attached to $\psi$. To make matters slightly more transparent, we have formulated it in algebraic form, as an extension of the pro-finite (and hence pro-algebraic) group $\Gamma_{F}$ by a complex reductive group, rather than an extension of the locally compact group $W_{F}$ by a compact topological group. For this reason we will work from now on with the Galois forms of $L$-groups rather than their Weil forms.

If $k=i$ is any index in $I_{\psi}$, we have the $L$-embedding

$$
\widetilde{\mu}_{i}=\xi_{\mu_{i}}:{ }^{L} H_{i} \longrightarrow{ }^{L}\left(G L\left(m_{i}\right)\right)=G L\left(m_{i}, \mathbb{C}\right) \times \Gamma_{F}
$$


that comes with $H_{i}$ as a (twisted) endoscopic datum for

$$
\widetilde{G}\left(m_{i}\right)=G L\left(m_{i}\right) \rtimes \widetilde{\theta}\left(m_{i}\right) .
$$

If $k=j$ belongs to $J_{\psi}$, we define an $L$-embedding

$$
\widetilde{\mu}_{j}:{ }^{L} H_{j} \longrightarrow{ }^{L}\left(G L\left(2 m_{j}\right)\right)=G L\left(2 m_{j}, \mathbb{C}\right) \times \Gamma_{F}
$$

by setting

$$
\tilde{\mu}_{j}\left(h_{j} \times \sigma\right)=\left(h_{j} \oplus \widetilde{\theta}_{j}\left(h_{j}\right)\right) \times \sigma, \quad h_{j} \in \widehat{H}_{j}=G L\left(m_{j}, \mathbb{C}\right), \sigma \in \Gamma_{F},
$$

where

$$
\widetilde{\theta}_{j}\left(h_{j}\right)=\widetilde{\theta}\left(m_{j}\right)\left(h_{j}\right)=\widetilde{J}\left(m_{j}\right) \cdot{ }^{t} h_{j}^{-1} \cdot \widetilde{J}\left(m_{j}\right)^{-1} .
$$

We then define an $L$-embedding

$$
\widetilde{\psi}: \mathcal{L}_{\psi} \times S L(2, \mathbb{C}) \longrightarrow{ }^{L}(G L(N))=G L(N, \mathbb{C}) \times \Gamma_{F}
$$

as the direct sum

$$
\widetilde{\psi}=\left(\bigoplus_{i \in I_{\psi}} \ell_{i}\left(\widetilde{\mu}_{i} \otimes \nu_{i}\right)\right) \oplus\left(\bigoplus_{j \in J_{\psi}} \ell_{j}\left(\widetilde{\mu}_{j} \otimes \nu_{j}\right)\right)
$$

Our use of $S L(2, \mathbb{C})$ here in place of $S U(2)$ is purely notational, and is in keeping with our construction of $\mathcal{L}_{\psi}$ as a complex pro-algebraic group. We are of course free to interpret the embedding $\widetilde{\psi}$ also as an $N$-dimensional representation of $\mathcal{L}_{\psi} \times S L(2, \mathbb{C})$. With either interpretation, we shall be primarily interested in the equivalence class of $\widetilde{\psi}$ as a $G L(N, \mathbb{C})$-conjugacy class of homomorphisms from $\mathcal{L}_{\psi} \times S L(2, \mathbb{C})$ to either $G L(N, \mathbb{C})$ or $G L(N, \mathbb{C}) \times \Gamma_{F}$.

We can now define the set of global parameters attached to any of our classical groups. Suppose that $G \in \widetilde{\mathcal{E}}_{\text {sim }}(N)$. We write $\widetilde{\Psi}(G)$ for the set of elements $\psi \in$ $\widetilde{\Psi}(N)$ such that $\widetilde{\psi}$ factors through ${ }^{L} G$. By this we mean that there exists an $L$ homomorphism

$$
\widetilde{\psi}_{G}: \mathcal{L}_{\psi} \times S L(2, \mathbb{C}) \longrightarrow{ }^{L} G
$$

such that

$$
\xi \circ \widetilde{\psi}_{G}=\widetilde{\psi},
$$

where $\xi$ is the $L$-embedding of ${ }^{L} G$ into ${ }^{L}(G L(N))$ that is part of the twisted endoscopic datum represented by $G$. Since $\widetilde{\psi}$ and $\xi$ are to be regarded as $G L(N, \mathbb{C})$ conjugacy classes of homomorphisms, $\widetilde{\psi}_{G}$ is determined up to the stabilizer in $G L(N, \mathbb{C})$ of its image, a group that contains $\widehat{G}$. The quotient of this stabilizer by $\widehat{G}$ equals the group $\widetilde{O}_{N}(G)$ of outer automorphisms of $\widehat{G}$ described in $\S 2$. It is trivial unless $G$ is of type $\mathbf{D}_{n}$, the case of an even orthogonal group in which $\widetilde{O}_{N}(G)$ equals $\mathbb{Z} / 2 \mathbb{Z}$. This case complicates our study of automorphic representations in a number of ways, all stemming from the fact that there can be two $\widehat{G}$-orbits of homomorphisms $\widetilde{\psi}_{G}$ in the larger class of $\widetilde{\psi}_{G}$. It is why we write $\widetilde{\Psi}(G)$ in place of the more familiar symbol $\Psi(G)$.

More generally, suppose that $G$ belongs to the larger set $\widetilde{\mathcal{E}}_{\text {ell }}(N)$ of elliptic data, or even the full set $\widetilde{\mathcal{E}}(N)$ of (twisted) endoscopic data for $\widetilde{G}(N)$. As a group over $F, G$ equals a direct product

$$
G=\prod_{\alpha} G_{\alpha}
$$


of groups $G_{\alpha}$ that range over (quasisplit) special orthogonal and symplectic groups and (split) general linear groups. We define the set of global parameters for $G$ as the product

$$
\widetilde{\Psi}(G)=\prod_{\alpha} \Psi_{\alpha}\left(G_{\alpha}\right),
$$

where $\Psi_{\alpha}\left(G_{\alpha}\right)$ equals $\widetilde{\Psi}\left(G_{\alpha}\right)$ if $G_{\alpha}$ is special orthogonal or symplectic, and equals $\Psi\left(G_{\alpha}\right)$ if $G_{\alpha}$ is a general linear group. It is not hard to see that an element in $\widetilde{\Psi}(G)$ can be identified with a pair $\left(\psi, \widetilde{\psi}_{G}\right)$, for a parameter $\psi \in \widetilde{\Psi}(N)$ and an $L$-homomorphism

$$
\widetilde{\psi}_{G}: \mathcal{L}_{\psi} \times S L(2, \mathbb{C}) \longrightarrow{ }^{L} G
$$

that satisfies (5.5), and is defined as a $\widehat{G}$-orbit only up to the action of $\widetilde{O u t}_{N}(G)$. The projection

$$
\left(\psi, \widetilde{\psi}_{G}\right) \longrightarrow \psi
$$

is not generally injective, in contrast to the injective embedding of $\widetilde{\Psi}(G)$ into $\widetilde{\Psi}(N)$ for simple $G$ that is an implicit part of our original definition. However, we still sometimes denote elements in the more general sets $\widetilde{\Psi}(G)$ by $\psi$ when there is no danger of confusion. In the global situation at hand, one is usually concerned only with the case that $G$ is elliptic, but the mapping is still not injective if $G$ is not simple.

Suppose that $\psi$ belongs to the set $\widetilde{\Psi}(G)$ we have just defined for any endoscopic datum $G \in \widetilde{\mathcal{E}}(N)$. We can then define the group

$$
S_{\psi}=S_{\psi}(G)=\operatorname{Cent}\left(\operatorname{im}\left(\widetilde{\psi}_{G}\right), \widehat{G}\right)
$$

we have been looking for as the centralizer of the image of $\widetilde{\psi}_{G}$. It is a reductive subgroup of $\widehat{G}$, whose quotient

$$
\mathcal{S}_{\psi}=\mathcal{S}_{\psi}(G)=S_{\psi} / S_{\psi}^{0} Z(\widehat{G})^{\Gamma_{F}}
$$

is a finite abelian 2-group. Notice that there is a canonical element

$$
s_{\psi}=\widetilde{\psi}_{G}\left(1,\left(\begin{array}{cc}
-1 & 0 \\
0 & -1
\end{array}\right)\right)
$$

in $S_{\psi}$. Its image in $\mathcal{S}_{\psi}$ plays a role in the classification of nontempered automorphic representations of $G$. We can also assign a twisted centralizer

$$
\widetilde{S}_{\psi}(N)=S_{\psi}(\widetilde{G}(N))=\operatorname{Cent}(\operatorname{im}(\widetilde{\psi}), G L(N, \mathbb{C}) \rtimes \theta)
$$

to $\psi$, as well as its untwisted analogue

$$
\widetilde{S}_{\psi}^{0}(N)=S_{\psi}\left(\widetilde{G}^{0}(N)\right)=\operatorname{Cent}(\operatorname{im}(\widetilde{\psi}), G L(N, \mathbb{C})) .
$$

Then $\widetilde{S}_{\psi}^{0}(N)$ is a product of complex general linear groups, embedded diagonally in $G L(N, \mathbb{C})$, as we noted in $\S 4$, and acts simply transitively by right or left translation on $\widetilde{S}_{\psi}(N)$. Since $\widetilde{S}_{\psi}(N)$ and $\widetilde{S}_{\psi}^{0}(N)$ are both connected, they do not complicate the automorphic representation theory of $G L(N)$.

Following $\S 3$, we write

$$
\widetilde{\Psi}_{\text {ell }}(N)=\Psi_{\text {ell }}(\widetilde{G}(N))
$$

for the subset of parameters $\psi \in \widetilde{\Psi}(N)$ such that the indexing set $J_{\psi}$ is empty, and such that $\ell_{i}=1$ for each $i \in I_{\psi}$. These objects are analogous to the self-dual representations $r$ we called elliptic in $\S 2$. Using the group $\mathcal{L}_{\psi} \times S L(2, \mathbb{C})$ in place 
of $\Lambda_{F}$, we can carry out the discussion of $\S 3$ without change here. Among other things, it tells us that any $\psi \in \widetilde{\Psi}_{\text {ell }}(N)$ has a unique source in one of the sets

$$
\widetilde{\Psi}(G), \quad G \in \widetilde{\mathcal{E}}_{\text {ell }}(N) .
$$

To be more precise, let $\widetilde{\Psi}_{2}(G)$ be the preimage of $\widetilde{\Psi}_{\text {ell }}(N)$ in $\widetilde{\Psi}(G)$, for any $G \in$ $\widetilde{\mathcal{E}}_{\text {ell }}(N)$. The mapping from $\widetilde{\Psi}(G)$ to $\widetilde{\Psi}(N)$ then takes $\widetilde{\Psi}_{2}(G)$ injectively ${ }^{10}$ onto a subset of $\widetilde{\Psi}_{\text {ell }}(N)$, which we identify with $\widetilde{\Psi}_{2}(G)$. Moreover, $\widetilde{\Psi}_{\text {ell }}(N)$ is a disjoint union

$$
\widetilde{\Psi}_{\text {ell }}(N)=\coprod_{G \in \widetilde{\mathcal{E}}_{\text {ell }}(N)} \widetilde{\Psi}_{2}(G)
$$

of these subsets. We thus have parallel chains of parameter sets

$$
\widetilde{\Psi}_{\text {sim }}(N) \subset \widetilde{\Psi}_{\text {ell }}(N) \subset \widetilde{\Psi}(N)
$$

and

$$
\widetilde{\Psi}_{\text {sim }}(G) \subset \widetilde{\Psi}_{2}(G) \subset \widetilde{\Psi}(G), \quad G \in \widetilde{\mathcal{E}}_{\text {ell }}(N),
$$

where $\widetilde{\Psi}_{\text {sim }}(G)$ denotes the intersection of $\widetilde{\Psi}_{\text {sim }}(N)$ with $\widetilde{\Psi}_{2}(G)$. Observe that $\widetilde{\Psi}_{2}(G)$ is the subset of parameters $\psi \in \widetilde{\Psi}(G)$ such that the centralizer $S_{\psi}$ is finite, while $\widetilde{\Psi}_{\text {sim }}(G)$ consists of those $\psi$ such that $S_{\psi}$ equals the minimal group $Z(\widehat{G})^{\Gamma_{F}}$.

The group $\mathcal{L}_{\psi}$ now seems quite promising. As we have just seen, it leads to the kind of objects we would attach to parameters defined on the product $L_{F} \times S U(2)$. In particular, we now have $L$-homomorphisms $\widetilde{\psi}$ and $\widetilde{\psi}_{G}$ from $\mathcal{L}_{\psi} \times S L(2, \mathbb{C})$ to groups ${ }^{L}(G L(N))$ and ${ }^{L} G$, with the corresponding centralizers $\widetilde{S}_{\psi}(N)$ and $S_{\psi}$ we will need. There is no denying that the process is pretty crude, starting with the ad hoc definition (5.3) that requires Theorem 5.1 as a long term induction hypothesis. It is also not appealing that $\mathcal{L}_{\psi}$ depends on the parameter $\psi$. Nevertheless, the group $\mathcal{L}_{\psi}$ does in the end serve our purpose. It is a kind of endoscopic hull of what would be the image of the Langlands group $L_{F}$ under a parameter $\psi$.

Much of our discussion here has been taken directly from $\S 1.4$ of [ECR]. The reader can refer to this section of $[\mathbf{E C R}]$ for some further discussion, having to do with the following natural questions:

(i) given $\psi \in \widetilde{\Psi}_{\text {ell }}(N)$, determine the unique $G \in \widetilde{\mathcal{E}}_{\text {ell }}(N)$ such that $\psi$ belongs to $\widetilde{\Psi}_{2}(G)$

(ii) more generally, given any $\psi \in \widetilde{\Psi}(N)$, determine all $G \in \widetilde{\mathcal{E}}_{\text {ell }}(N)$ such that $\psi$ lies in the image of $\widetilde{\Psi}(G)$;

(iii) given $G \in \widetilde{\mathcal{E}}_{\text {sim }}(N)$ and $\psi \in \widetilde{\Psi}(G)$, determine the centralizer $S_{\psi}=S_{\psi}(G)$ explicitly.

However, there is another point from [ECR, $\S 1.4]$ that we do need to mention here. It concerns a bijective correspondence, which is elementary, but which is also in some sense the theoretical center of the theory of endoscopy.

In $\S 3$, we described endoscopic data $G^{\prime} \in \mathcal{E}(G)$ for $G$. Since any such $G^{\prime}$ is again a direct product

$$
G^{\prime}=\prod_{\alpha^{\prime}} G_{\alpha^{\prime}}^{\prime}
$$

\footnotetext{
${ }^{10}$ so in particular, the mapping from $\widetilde{\Psi}(G)$ to $\widetilde{\Psi}(N)$ that we agreed above was not injective if $G$ lies in the complement of $\widetilde{\mathcal{E}}_{\text {sim }}(N)$ in $\widetilde{\mathcal{E}}_{\text {ell }}(N)$, is injective upon restriction to the subset $\widetilde{\Psi}_{2}(G)$ of $\widetilde{\Psi}(G)$.
} 
of groups of the kind we have studied, we can define the set of parameters

$$
\widetilde{\Psi}\left(G^{\prime}\right)=\prod_{\alpha^{\prime}} \Psi\left(G_{\alpha^{\prime}}^{\prime}\right)
$$

as above. We can also form the centralizer group $S_{\psi^{\prime}}=S_{\psi^{\prime}}\left(G^{\prime}\right)$, for any $\psi^{\prime}$ in this set.

Consider a pair

$$
\left(G^{\prime}, \psi^{\prime}\right), \quad G^{\prime} \in \mathcal{E}(G), \psi^{\prime} \in \widetilde{\Psi}\left(G^{\prime}\right) .
$$

We recall that $G^{\prime}$ really represents an isomorphism class of triplets $\left(G^{\prime}, s^{\prime}, \xi^{\prime}\right)$, where $s^{\prime}$ is a semisimple element in $\widehat{G}^{\prime}$ and $\xi^{\prime}$ is an $L$-embedding of ${ }^{L} G^{\prime}$ into ${ }^{L} G$. The parameter $\psi^{\prime}=\psi_{G^{\prime}}$ can be identified with a pair $\left(\psi, \widetilde{\psi}^{\prime}\right)$, for a parameter $\psi \in \widetilde{\Psi}(G)$ and an $L$-embedding

$$
\widetilde{\psi}^{\prime}=\widetilde{\psi}_{G^{\prime}}: \mathcal{L}_{\psi} \times S L(2, \mathbb{C}) \longrightarrow{ }^{L} G^{\prime}
$$

such that

$$
\xi^{\prime} \circ \widetilde{\psi}^{\prime}=\widetilde{\psi}_{G}
$$

in the notation above, where $\widetilde{\psi}^{\prime}$ is defined as a $\widehat{G}^{\prime}$-orbit only up to the action of the finite group

$$
\widetilde{\operatorname{Out}}_{N}\left(G^{\prime}\right)=\operatorname{Out}_{G}\left(G^{\prime}\right) \times \widetilde{\operatorname{Out}}_{N}(G) .
$$

The pair $\left(G^{\prime}, \psi^{\prime}\right)$ gives rise to a second pair

$$
(\psi, s), \quad \psi \in \widetilde{\Psi}(G), s \in \mathcal{E}\left(\bar{S}_{\psi}\right),
$$

where $\mathcal{E}\left(\bar{S}_{\psi}\right)=\mathcal{E}\left(\bar{S}_{\psi, \text { ss }}\right)$ denotes the set of semisimple conjugacy classes in the complex reductive group $\bar{S}_{\psi}=S_{\psi} / Z(\widehat{G})^{\Gamma_{F}}$. Indeed $\psi=\psi_{G}$ is attached to $\psi^{\prime}$ as above, while $s$ is just the image $\xi^{\prime}\left(s^{\prime}\right)$ of $s^{\prime}$ in $\widehat{G}$.

Conversely, suppose that it is a pair $(\psi, s)$ of the second sort (5.10) that we are given. With this information, we set $\widehat{G}^{\prime}$ equal to the connected centralizer of $s$ in $\widehat{G}$, and $s^{\prime}$ equal (somewhat superfluously) to the preimage of $s$ in $\widehat{G}^{\prime}$. The product

$$
\mathcal{G}^{\prime}=\widehat{G}^{\prime} \cdot \widetilde{\psi}_{G}\left(\mathcal{L}_{\psi} \times S L(2, \mathbb{C})\right)
$$

of $\widehat{G}^{\prime}$ with the image of $\widetilde{\psi}_{G}$ can be identified with an $L$-subgroup of ${ }^{L} G$, for which the identity embedding $\xi^{\prime}$ is an $L$-homomorphism. We define $G^{\prime}$ to be a quasisplit group for which $\widehat{G}^{\prime}$, equipped with the $L$-action induced by $\mathcal{G}^{\prime}$, is a dual group. The triplet $\left(G^{\prime}, s^{\prime}, \xi^{\prime}\right)$ represented by $G^{\prime}$ is then an endoscopic datum for $G$, as defined in $\S 3$. Since $s$ lies in the centralizer of the image of the $L$-embedding $\widetilde{\psi}_{G}$ attached to $\psi, \widetilde{\psi}_{G}$ factors through ${ }^{L} G^{\prime}$. We obtain an $L$-embedding $\widetilde{\psi}^{\prime}$ of $\mathcal{L}_{\psi} \times S L(2, \mathbb{C})$ into ${ }^{L} G^{\prime}$ that satisfies (5.9), and hence, an element $\psi^{\prime} \in \widetilde{\Psi}\left(G^{\prime}\right)$. The pair $(\psi, s)$ thus leads in the other direction to a pair $\left(G^{\prime}, \psi^{\prime}\right)$ of the first sort (5.8).

The bijective correspondence

$$
\left(G^{\prime}, \psi^{\prime}\right) \longrightarrow(\psi, s)
$$

is a general phenomenon. It applies to arbitrary endoscopic data, twisted or otherwise, and corresponding spectral parameters. In particular, it has a natural variant in the case that $\left(G, G^{\prime}\right)$ is replaced by $(\widetilde{G}(N), G)$. It also applies without change if $F$ is replaced by a local field. In all cases, the correspondence transforms questions on the transfer of characters to questions on the groups $S_{\psi}$. 
Finally, we will need to know how to localize global parameters for $G$. For any $v$, we have the local parameter set $\Psi\left(G_{v}\right)$ defined in $\S 4$. We write $\Psi^{+}\left(G_{v}\right)$ for the larger set of parameters on $L_{F_{v}} \times S U(2)$, in which the image in $\widehat{G}$ is not required to be bounded. To match our global convention, we will also write $\widetilde{\Psi}^{+}\left(G_{v}\right)$ for the quotient of $\Psi^{+}\left(G_{v}\right)$ by the group $\widetilde{O u t}_{N}\left(G_{v}\right)$. We would like to show that for any $G \in \widetilde{\mathcal{E}}_{\text {sim }}(N)$, the mapping (4.12) from $\Psi(N)$ to $\Psi_{v}^{+}(N)$ takes the subset $\widetilde{\Psi}(G)$ of $\Psi(N)$ into the subset $\widetilde{\Psi}^{+}\left(G_{v}\right)$ of $\Psi_{v}^{+}(N)$. This property is not elementary. It is a consequence of a second "seed theorem", which we state as a complement to Theorem 5.1, but which like Theorem 5.1, has in the end to be proved at the same time as broader theorems.

THEOREM 5.2. Suppose that $\phi \in \widetilde{\Phi}_{\text {sim }}(N)$ is simple generic, as in Theorem 5.1 . Then the localization $\phi_{v}$ of $\phi$ at any $v$, a priori an element in the subset $\widetilde{\Phi}_{v}(N)$ of local generic parameters in $\widetilde{\Psi}_{v}^{+}(N)$, lies in the subset $\widetilde{\Phi}\left(G_{\phi, v}\right)$ of $\widetilde{\Phi}_{v}(N)$ attached to the localization $G_{\phi, v}$ of the global datum $G_{\phi} \in \widetilde{\mathcal{E}}_{\text {sim }}(N)$ of Theorem 5.1.

The theorem asserts that the $N$-dimensional representation $\phi_{v}$ of $L_{F_{v}}$, which is attached by the local Langlands correspondence to the cuspidal automorphic representation of $G L(N)$ given by $\phi$, factors through the local endoscopic embedding

$$
\xi_{\phi, v}:{ }^{L} G_{\phi, v} \longrightarrow{ }^{L}\left(G L(N)_{v}\right) .
$$

It allows us to identify $\phi_{v}$ with a local $L$-homomorphism from $L_{F_{v}}$ to ${ }^{L} G_{\phi, v}$. Like its global companion Theorem 5.1, Theorem 5.2 is proved by a long induction argument that includes the proof of broader theorems. Since we will not be able to present the general argument here, we will just assume both Theorems 5.1 and 5.2 in what follows.

We apply Theorem 5.2 to each of the orthogonal and symplectic factors ${ }^{L} H_{k}$ in the fibre product (5.3). Putting them together, and applying only the local correspondence of Theorem 4.4 in case $H_{k}=G L\left(m_{k}\right)$, we obtain a conjugacy class of $L$-homomorphisms

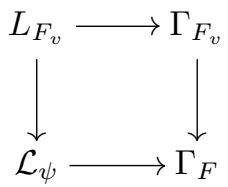

It is determined up the action of the group

$$
\widetilde{\operatorname{Out}}\left(\mathcal{L}_{\psi}\right)=\prod_{k} \widetilde{\mathrm{Out}}_{m_{k}}\left(H_{k}\right)
$$

where $\widetilde{O} u_{m_{k}}\left(H_{k}\right)=1$ in case $H_{k}=G L\left(m_{k}\right)$. This is the analogue of (2.3) for our makeshift substitute $\mathcal{L}_{\psi}$ for $L_{F}$.

Suppose that $\psi$ belongs to $\widetilde{\Psi}(G)$ for some $G \in \widetilde{\mathcal{E}}_{\text {sim }}(N)$, or indeed, for any $G$ in the general set $\widetilde{\mathcal{E}}(N)$. It then follows from this discussion that we can identify the localization of $\psi$, as an element in $\widetilde{\Psi}_{v}^{+}(N)$, with an $L$-homomorphism

$$
\psi_{v}: L_{F_{v}} \times S U(2) \longrightarrow{ }^{L} G_{v} .
$$


This fits into a larger commutative diagram of $L$-homomorphisms

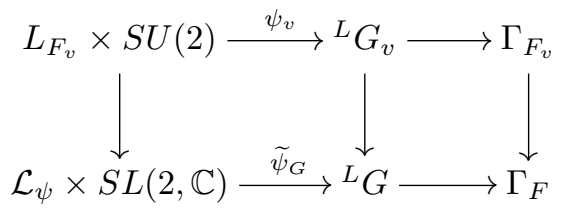

where the left hand vertical arrow is given by the mapping of $L_{F_{v}}$ into $\mathcal{L}_{\psi}$ in (5.12), and the embedding of $S U(2)$ into $S L(2, \mathbb{C})$. In particular, we obtain a localization mapping of the form (4.12) that takes $\widetilde{\Psi}(G)$ into the set $\widetilde{\Psi}^{+}\left(G_{v}\right)$. Moreover, since $\psi_{v}$ is essentially the restriction of the global embedding $\widetilde{\psi}_{G}$ to the image of $L_{F_{v}} \times S U(2)$, the global centralizer $S_{\psi}$ is contained in $S_{\psi_{v}}$. From this, it follows that there is a canonical mapping

$$
x \longrightarrow x_{v}, \quad x \in \mathcal{S}_{\psi},
$$

of $\mathcal{S}_{\psi}$ into $\mathcal{S}_{\psi_{v}}$.

\section{Transfer and the fundamental lemma}

The field $F$ will be local in this section unless stated otherwise. The essential problem is to establish the local Langlands correspondence for a special orthogonal or symplectic group $G \in \widetilde{\mathcal{E}}_{\text {sim }}(G)$. It is closely related to local functoriality, specifically the second of two cases described at the end of $\S 1$, in which $G^{\prime} \in \widetilde{\mathcal{E}}(G)$ is an endoscopic group for $G$. Within the general principle of functoriality, this case is distinguished by being also a part of the separate theory of endoscopy. As such, it should come with a characterization of the image of the functorial correspondence

$$
\pi^{\prime} \longrightarrow \pi, \quad \pi^{\prime} \in \Pi\left(G^{\prime}\right),
$$

of Principle 1.1. The reason for this is that the functorial correspondence of representations will be dual to a transfer of functions from $G(F)$ to $G^{\prime}(F)$.

The transfer of functions is based on harmonic analysis. Its domain is a space of test functions on $G(F)$, such as the space $C_{c}^{\infty}(G)$ of functions on $G(F)$ that are smooth (which means infinitely differentiable if $F$ is archimedean, and locally constant if $F$ in nonarchimedean) and compactly supported. Following [ECR], we will instead take the Hecke algebra $\mathcal{H}(G)$, a convolution algebra that equals $C_{c}^{\infty}(G)$ if $F$ is nonarchimedean, but that is the proper subalgebra of functions $f \in C_{c}^{\infty}(G)$ that satisfy a supplementary finiteness condition under left and right translation of $f$ by elements in a fixed maximal compact subgroup of $G(F)$, if $F$ is archimedean.

An element $\gamma \in G(F)$ is called strongly regular if its centralizer

$$
G_{\gamma}=\operatorname{Cent}(\gamma, G)=\left\{x \in G: x^{-1} \gamma x=\gamma\right\}
$$

is a (maximal) torus in $G$. For any such $\gamma$, we have the associated invariant orbital integral

$$
f_{G}(\gamma)=|D(\gamma)|^{\frac{1}{2}} \int_{G_{\gamma}(F) \backslash G(F)} f\left(x^{-1} \gamma x\right) d x
$$

of any test function $f \in \mathcal{H}(G)$, where $d x$ is a fixed, right invariant measure on the coset space $G_{\gamma}(F) \backslash G(F)$. We have normalized $f_{G}(\gamma)$ by the Weyl discriminant

$$
D(\gamma)=\operatorname{det}\left((1-\operatorname{Ad}(\gamma))_{\mathfrak{g} / \mathfrak{g}_{\gamma}}\right)
$$


where $\mathfrak{g}$ and $\mathfrak{g}_{\gamma}$ are the Lie algebras of $G$ and $G_{\gamma}$. We regard $f_{G}$ as a function on the set of strongly regular points $\gamma$, and write

$$
\mathcal{I}(G)=\left\{f_{G}: f \in \mathcal{H}(G)\right\}
$$

for the image of $\mathcal{H}(G)$ under this transform.

The functions in $\mathcal{I}(G)$ also have a spectral interpretation. Any representation $\pi \in \Pi(G)$ has a character, which can be identified with the linear form

$$
\operatorname{tr}(\pi(f))=\operatorname{tr}\left(\int_{G(F)} f(x) \pi(x) d x\right), \quad f \in \mathcal{H}(G),
$$

on $\mathcal{H}(G)$. We set

$$
f_{G}(\pi)=\operatorname{tr}(\pi(f))
$$

in analogy with the notation we have used for the dual orbital integrals. It can then be shown that either of the two functions $\left\{f_{G}(\gamma)\right\}$ or $\left\{f_{G}(\pi)\right\}$ attached to $f$ determines the other. We can therefore regard any element $f_{G}$ in $\mathcal{I}(G)$ as a function of either $\gamma$ or $\pi$. It is invariant, in the sense that it depends only on the conjugacy class of $\gamma$ or the equivalence class of $\pi$. It also remains invariant if its preimage $f \in \mathcal{H}(G)$ is replaced by any conjugate

$$
f^{y}(x)=f\left(y x y^{-1}\right), \quad x, y \in G(F) .
$$

The theory of endoscopy is founded on the fact that conjugacy in $G(F)$ is finer than geometric conjugacy. Two strongly regular elements in $G(F)$ are said to be stably conjugate if they are conjugate as elements in the group $G(\bar{F})$ of geometric points in $G$. For the local field $F$, it is easy to show that there are only finitely many $G(F)$-conjugacy classes $\gamma$ in any (strongly regular) stable conjugacy class $\delta$. The corresponding sum

$$
f^{G}(\delta)=\sum_{\gamma} f_{G}(\gamma), \quad f \in \mathcal{H}(G),
$$

of orbital integrals is called the stable orbital integral of the given function $f$ at $\delta$. We write

$$
\mathcal{S}(G)=\left\{f^{G}: f \in \mathcal{H}(G)\right\}
$$

for the space of functions of $\delta$ obtained in this way. As we shall see, $L$-packets arise when we try to find a spectral interpretation for the functions $f^{G}$ in $\mathcal{S}(G)$ analogous to the values $f_{G}(\pi)$ of functions $f_{G}$ in $\mathcal{I}(G)$. In general, a distribution on $G(F)$, or more correctly a continuous linear form on $\mathcal{H}(G)$, is said to be stable if its value at any $f$ depends only on $f^{G}$. If this is so, we can identify $S$ with the linear form

$$
\widehat{S}\left(f^{G}\right)=S(f), \quad f \in \mathcal{H}(G),
$$

on $\mathcal{S}(G)$. The spectral question above is then to attach stable distributions to representations $\pi$.

Suppose that $G^{\prime}$ is an endoscopic datum for $G$. Langlands and Shelstad [LS] define a strongly regular element $\delta^{\prime}$ in $G^{\prime}(F)$ to be strongly $G$-regular if its image in $G(F)$ (under any admissible embedding $[\mathbf{L S},(1.3)]$ of its centralizer $G_{\delta^{\prime}}^{\prime}$ into $G$ ) is strongly regular for $G$. The space of strongly $G$-regular elements remains open and dense in $G^{\prime}(F)$, so functions in the space $\mathcal{S}\left(G^{\prime}\right)$ are determined by their values on strongly $G$-regular, stable conjugacy classes in $G^{\prime}(F)$. The point of the article $[\mathbf{L S}]$ was to introduce an explicit function $\Delta\left(\delta^{\prime}, \gamma\right)$ of a strongly $G$-regular stable conjugacy class $\delta^{\prime}$ in $G^{\prime}(F)$ and a strongly regular conjugacy class $\gamma$ in $G(F)$, 
which they called a transfer factor for $G$ and $G^{\prime}$. By construction, this function vanishes unless $\gamma$ belongs to the stable conjugacy class of the image of $\delta^{\prime}$ in $G^{\prime}(F)$. It therefore has finite support in either of the variables when the complementary variable is fixed. The role of $\Delta\left(\delta^{\prime}, \gamma\right)$ is as the kernel function for the transfer mapping that sends a function $f \in \mathcal{H}(G)$ to the function

$$
f^{\prime}\left(\delta^{\prime}\right)=f_{\Delta}^{G^{\prime}}\left(\delta^{\prime}\right)=\sum_{\gamma} \Delta\left(\delta^{\prime}, \gamma\right) f_{G}(\gamma)
$$

of $\delta^{\prime}$. Langlands and Shelstad conjectured that the function $f^{\prime}\left(\delta^{\prime}\right)$ belongs to the space $\mathcal{S}\left(G^{\prime}\right)$.

The Langlands-Shelstad transfer conjecture remained a fundamental problem for twenty years. It had been established earlier for archimedean $F$ (and ad hoc transfer factors) by Shelstad [She1]. But for nonarchimdean $F$, it was closely tied to the fundamental lemma. The fundamental lemma is a related conjecture, posed originally by Langlands, which became precise with the introduction of the transfer factors of $[\mathbf{L S}]$. It applies to the case that the quasisplit groups $G$ and $G^{\prime}$ are unramified, which means that they are both split over an unramified extension of the nonarchimedean field $F$. With this condition, $G(F)$ has a hyperspecial maximal compact subgroup $K_{F}$, an important object determined uniquely up to the appropriate analogue of stable conjugacy. The fundamental lemma asserts that if $f$ is the characteristic function of $K_{F}$ (a function in $\mathcal{H}(G)$ since $K_{F} \subset G(F)$ is open), then $f^{\prime}$ equals the image in $\mathcal{S}\left(G^{\prime}\right)$ of the characteristic function of any hyperspecial maximal compact subgroup $K_{F}^{\prime}$ of $G^{\prime}(F)$. It thus represents a more precise version of the transfer conjecture in a special case.

Kottwitz and Shelstad [KS] later extended the results of $[\mathbf{L S}]$ to twisted endoscopic data. They had a number of new problems to deal with, but for our setting here, we need include only a small modification of the discussion above. To do so, we replace the group $G$ by the component $\widetilde{G}(N)=G L(N) \rtimes \widetilde{\theta}(N)$, and its endoscopic datum $G^{\prime}$ by a twisted endoscopic datum $G \in \widetilde{\mathcal{E}}(N)$. Endoscopic transfer in this setting is again tied to local functoriality, this time to the first of the two cases introduced at the end of $\S 1$. From what we have just said, it is clear that this case is also distinguished by being part of the separate theory of endoscopy.

One defines the Hecke module $\widetilde{\mathcal{H}}(N)=\mathcal{H}(\widetilde{G}(N))$ of functions $\widetilde{f}$ on $\widetilde{G}(N, F)$, and the notion of a strongly regular element $\widetilde{\gamma}$ in $\widetilde{G}(N, F)$. One then defines the twisted orbital integral $\widetilde{f}_{N}(\widetilde{\gamma})$ of $\widetilde{f}$ over the orbit of $\widetilde{\gamma}$ under the group $\widetilde{G}^{0}(N, F)=$ $G L(N, F)$ acting by conjugation on $\widetilde{G}(N, F)$. The twisted transfer factor from $[\mathbf{K S}]$ is an explicit, but sophisticated function $\widetilde{\Delta}(\delta, \widetilde{\gamma})$ of a strongly $\widetilde{G}(N)$-regular, stable conjugacy class $\delta$ in $G(F)$ and a strongly regular, twisted conjugacy class $\widetilde{\gamma}$ in $\widetilde{G}(N, F)$. It serves as the kernel function for the transfer mapping that sends a function $\tilde{f} \in \widetilde{\mathcal{H}}(N)$ to the function

$$
\tilde{f}^{G}(\delta)=\tilde{f}_{\widetilde{\Delta}}^{G}(\delta)=\sum_{\widetilde{\gamma}} \widetilde{\Delta}(\delta, \widetilde{\gamma}) \tilde{f}_{N}(\widetilde{\gamma})
$$

of $\delta$. For reasons similar to those above, the sum can again be taken over a finite set that depends only on $\delta$. Folowing $[\mathbf{L S}]$, Kottwitz and Shelstad conjectured that the function $\widetilde{f}^{G}(\delta)$ belongs to the space $\mathcal{S}(G)$. The twisted fundamental lemma asserts that if $G$ is unramified, and $\widetilde{f}$ is the characteristic function of the open 
subset $G L\left(N, \mathfrak{o}_{F}\right) \rtimes \widetilde{\theta}(N)$ of $\widetilde{G}(N, F), \widetilde{f}^{G}$ is the image in $\mathcal{S}(G)$ of the characteristic function of a hyperspecial, maximal compact subgroup $K_{F}$ of $G(F)$.

For archimedean $F$, Shelstad has recently completed a proof of the general twisted transfer conjecture [She5], using the explicit specialization to real groups of the twisted transfer factors of [KS]. This followed other recent papers [She2][She4], in which she reformulated much of her earlier work on ordinary (untwisted) endoscopy from the perspective of $[\mathbf{L S}]$.

For nonarchimedean $F$, the breakthrough was the geometric proof of the fundamental lemma by Ngô [N]. He combined the local geometric ideas of Goresky, Kottwitz and MacPherson $[\mathbf{G K M}]$ on affine Springer fibres with an analogue of the global Hitchin fibration to establish the fundamental lemma for a local field of positive characteristic. By earlier results on the independence of characteristic [W2], this gave the fundamental lemma also for the local field $F$ of characteristic 0. The paper of Ngô treats the ordinary (untwisted) fundamental lemma, and a variant to which Waldspurger had reduced the general (twisted) case [W3]. It therefore resolves the fundamental lemma in complete generality. As for the KottwitzLanglands-Shelstad (KLS) transfer conjecture, Waldspurger had established some time ago that the ordinary transfer conjecture would follow from the fundamental lemma [W1]. His more recent papers [W3], [W4] extend this implication to the general case. The general results of Waldspurger therefore yield the nonarchimedean LSK-conjecture in all cases.

We will use the transfer mapping from $\widetilde{G}(N)$ to answer the question posed above on a spectral interpretation for the functions in $\mathcal{S}(G)$. We begin with a local parameter $\psi \in \Psi(N)$ for $G L(N)$. From the correspondence (4.11) derived from Theorem 4.4, we obtain a irreducible unitary representation $\pi_{\psi} \in \Pi_{\text {unit }}(N)$ of $G L(N, F)$. Assume that $\psi$ lies in the subset $\widetilde{\Psi}(N)$ of self-dual parameters. As we have noted, $\pi_{\psi}$ then has an extension to the group $\widetilde{G}(N, F)^{+}$. However, the extension is determined a priori only up to the sign character on the semidirect factor $\widetilde{\theta}(N)^{+}$of $\widetilde{G}(N, F)^{+}$. We need to define it uniquely.

It is the theory of Whittaker models that provides a canonical extension of $\pi_{\psi}$. This theory is well understood for general linear groups, and is expected to carry over to tempered $L$-packets for general quasisplit groups. In fact, for our group $G$, the conjectured properties (proposed by Shahidi in [Sha]) were established in $\S 8.3$ of [ECR]. In general, one must fix a Whittaker datum $(B, \chi)$, consisting of a rational Borel subgroup $B$ of a given quasisplit group over $F$, and a nondegenerate character $\chi$ on the unipotent radical $N_{B}(F)$ of $B(F)$. (In the case of a twisted group, such as $\widetilde{G}(N)$, one must take $(B, \chi)$ to be stable under the relevant automorphism, $\widetilde{\theta}(N)$ in the case $\widetilde{G}(N)$.) A $(B, \chi)$-Whittaker vector for an irreducible representation is then a nonzero vector in the underlying complex vector space, on which $N_{B}(F)$ is $\chi$-equivariant. If it exists for the given representation, a $(B, \chi)$-Whittaker vector is known to be unique up to a complex multiple. (See the brief introduction in $[\mathbf{E C R}$, $\S 2.5]$, for example.)

We will not review the theory of Whittaker models further, except to note its role in the choice of transfer factors. In general, a transfer factor is defined uniquely only up to a nonzero scalar multiple. But for our group $G$ (and indeed, for any quasisplit group over $F$ ), Langlands and Shelstad attach a canonical transfer factor to any $F$-splitting $[\mathbf{L S}, \S 1.3]$ of $G$. The group $G_{\text {ad }}(F)$ of $F$-points in the adjoint group of $G$ acts simply transitively on the set of $F$-splittings of $G$. The associated 
transfer factors are equal (for all $G^{\prime}$ ) if and only if their splittings lie in the same orbit under the image $(G(F))_{\text {ad }}$ of $G(F)$ in $G_{\text {ad }}(F)$. The finite quotient

$$
G_{\text {ad }}(F) /(G(F))_{\text {ad }}
$$

therefore acts simply transitively on the set of (families of) normalized transfer factors. These normalizations are really geometric, in that they lead to the simplest explicit formulas for the transfer of orbital integrals. Kottwitz and Shelstad, for their part, introduced a different normalization for the transfer factors of $G$ (and for any quasisplit group over $F$ ). It was attached to any Whittaker datum for $G$. The group $G_{\text {ad }}(F)$ also acts simply transitively on the set of Whittaker data, and the quotient (6.6) again acts simply transitively on the corresponding set of such normalizations. These are spectral normalizations, in that they are expected to lead to the simplest explicit formulas for the transfer of characters. We assume implicitly from now on that the transfer factors for $G$ have been assigned the Whittaker normalization attached to a fixed Whittaker datum.

Similar remarks apply to twisted transfer factors, but there is no need to discuss them explicitly. We simply fix a $\widetilde{\theta}(N)$-stable Whittaker datum $(\widetilde{B}(N), \widetilde{\chi}(N))$ for $G L(N)$, and work with the associated normalized twisted transfer factors. If $\psi=\phi$ lies in the subset $\widetilde{\Phi}_{\text {bdd }}(N)$ of $\widetilde{\Psi}(N)$, the self-dual representation $\pi_{\psi}=\pi_{\phi}$ of $G L(N, F)$ is tempered, and therefore has a $(\widetilde{B}(N), \widetilde{\chi}(N))$-Whittaker vector. We take $\widetilde{\pi}_{\phi}$ to be the unique extension of $\pi_{\phi}$ to the group $\widetilde{G}(N, F)^{+}$such that the operator $\widetilde{\pi}_{\phi}(N)=\widetilde{\pi}_{\phi}(\widetilde{\theta}(N))$ maps the Whittaker vector to itself. The definition carries over to our general parameter $\psi \in \widetilde{\Psi}(N)$, even though the nontempered Speh representation $\pi_{\psi}$ need not have a Whittaker vector. For we can work with the induced representation $\rho_{\psi}$ of which $\pi_{\psi}$ is the Langlands quotient. This representation does have a Whittaker vector, which serves to define an extension $\widetilde{\rho}_{\psi}$ of $\rho_{\psi}$. Its quotient then gives an extension $\widetilde{\pi}_{\psi}$ of $\pi_{\psi}$. (See [ECR, §2.1].)

Given the extension $\widetilde{\pi}_{\psi}$ of $\pi_{\psi}$, we define a linear form

$$
\tilde{f}_{N}(\psi)=\operatorname{tr}\left(\widetilde{\pi}_{\psi}(\widetilde{f})\right), \quad \tilde{f} \in \tilde{\mathcal{H}}(N),
$$

on $\widetilde{\mathcal{H}}(N)$. Does it transfer to $G$ ? More precisely, is it the image of a stable linear form on $\mathcal{H}(G)$ that is dual to the transfer $\widetilde{f} \rightarrow \widetilde{f}^{G}$ of functions? We would expect a necessary condition to be that as an $L$-homomorphism from $L_{F} \times S U(2)$ into ${ }^{L}(G L(N)), \psi$ factors through the $L$-subgroup ${ }^{L} G$. In other words, $\psi$ should lie in the subset

$$
\widetilde{\Psi}(G)=\Psi(G) / \operatorname{Out}_{N}(G)
$$

of $\widetilde{\Psi}(N)$ attached to our group $G \in \widetilde{\mathcal{E}}_{\text {sim }}(N)$. Now the dual transfer of functions takes $\widetilde{\mathcal{H}}(N)$ into the subspace $\widetilde{\mathcal{S}}(G)$ of functions in $\mathcal{S}(G)$ that are invariant under the finite group $\widetilde{\mathrm{Out}_{N}}(G)$. It is convenient also to write $\widetilde{\mathcal{H}}(G)$ for the subspace of functions in $\mathcal{H}(G)$ invariant under $\operatorname{Out}_{N}(G)$ (with the nontrivial element in $\operatorname{Out}_{N}(G)$, when it exists, identified as in $\S 3$ with an actual $F$-automorphism of $G)$. The question above should then be whether (6.7) transfers to a stable linear form on the subspace $\widetilde{\mathcal{H}}(G)$ of $\mathcal{H}(G)$. The following theorem, which is a foundation for the local correspondence we will state in the next section, gives an affirmative answer. 
TheOREM 6.1. Suppose that $F$ is local, that $G \in \widetilde{\mathcal{E}}_{\mathrm{ell}}(N)$, and that $\psi$ lies in the set $\widetilde{\Psi}(G)$ attached to a fixed group $G \in \widetilde{\mathcal{E}}_{\mathrm{ell}}(N)$. Then there is a unique stable linear form

$$
f \longrightarrow f^{G}(\psi), \quad f \in \widetilde{\mathcal{H}}(G),
$$

on $\widetilde{\mathcal{H}}(G)$ with the general property

$$
\widetilde{f}^{G}(\psi)=\widetilde{f}_{N}(\psi), \quad \tilde{f} \in \tilde{\mathcal{H}}(N),
$$

together with the secondary property

$$
f^{G}(\psi)=f^{S}\left(\psi_{S}\right) f^{O}\left(\psi_{O}\right), \quad \tilde{f} \in \tilde{\mathcal{H}}(G),
$$

in case

$$
\begin{array}{ll}
G=G_{S} \times G_{O}, \quad G_{\varepsilon} \in \widetilde{\mathcal{E}}_{\operatorname{sim}}\left(N_{\varepsilon}\right), \\
\psi=\psi_{S} \times \psi_{O}, \quad \psi_{\varepsilon} \in \widetilde{\Psi}\left(G_{\varepsilon}\right),
\end{array}
$$

and

$$
f^{G}=f^{S} \times f^{O}, \quad f^{\varepsilon} \in \widetilde{\mathcal{S}}\left(G_{\varepsilon}\right), \varepsilon=O, S,
$$

are composite.

REMARKs. 1 . The primary case is for $G \in \widetilde{\mathcal{E}}_{\text {sim }}(N)$ simple. It has dominated our past discussion, for the reason that many questions for composite twisted endoscopic data are amenable to induction. When $G$ is simple, the mapping $\widetilde{f} \rightarrow \widetilde{f}^{G}$ takes $\widetilde{\mathcal{H}}(N)$ onto $\widetilde{\mathcal{S}}(G)$ [ECR, Corollary 2.2]. The uniqueness of the linear form (6.8) then follows from the formula (6.9) in this case.

2. If $G \in \widetilde{\mathcal{E}}_{\text {ell }}(N)$ is composite, the uniqueness follows from the product formula (6.10). In this case, the symbol $\psi$ on the right hand side of (6.9) is understood to be the image of the given composite parameter under the (not necessarily injective) mapping from $\widetilde{\Psi}(G)$ to $\widetilde{\Psi}(N)$. The problem here is to establish the compatibility condition represented by the two sides of (6.9).

3 . Suppose that $\psi$ lies in the set $\widetilde{\Psi}(G)$ attached to some $G$ in the complement of $\widetilde{\mathcal{E}}_{\text {ell }}(N)$ in the full set $\widetilde{\mathcal{E}}(N)$ of twisted endoscopic data. The assertions (6.8) and (6.9) of the theorem then follow easily by reduction to the Levi component of a proper, $\widetilde{\theta}(N)$-stable parabolic subgroup of $G L(N)$.

4. The notation $f^{G}(\psi)$ in (6.8) is deliberate, even if perhaps also slightly confusing. It reminds us that we are dealing with a linear form on $\widetilde{\mathcal{S}}(G)$. In particular, we can define $f^{G}(\phi)$ for any $\phi$ in the subset $\widetilde{\Phi}_{\text {bdd }}(G)$ of $\widetilde{\Psi}(G)$, or by analytic continuation, any $\phi$ in the larger set $\widetilde{\Phi}(G)$. It can be shown that either of the two functions $\left\{f^{G}(\delta)\right\}$ or $\left\{f^{G}(\phi)\right\}$ attached to $f$ determines the other. We can therefore regard any element $f^{G}$ in $\widetilde{\mathcal{S}}(G)$ as a function of either $\delta$ or $\phi$. This is in answer to the question above on a spectral interpretation of the function $f^{G}$ (or rather, the slightly weaker question for the subspace $\widetilde{\mathcal{S}}(G)$ of $\mathcal{S}(G)$ ). The local classification theorem, which we state in the next section, expresses the stable character (6.8) of $\phi$ in terms of the characters of representations $\pi$ in the $L$-packet of $\phi$, in spectral analogy with the conjugacy classes $\gamma$ in a stable conjugacy class $\delta$. 


\section{Statement of theorems}

We will now state our theorems of classification. We fix a (quasisplit, special) orthogonal or symplectic group $G \in \widetilde{\mathcal{E}}_{\text {sim }}(N)$ over the field $F$. Our ultimate concern is the global classification of automorphic representations. However, this necessarily relies on an understanding of local irreducible representations. We therefore assume for the moment that $F$ is local.

We would ideally like to attach a canonical $L$-packet $\Pi_{\phi}$ of irreducible representations $\pi \in \Pi(G)$ of $G(F)$ to any local Langlands parameter $\phi \in \Phi(G)$. As we shall explain presently, it would suffice to consider the case of bounded parameters $\phi \in \Phi_{\text {bdd }}(G)$, which leads to $L$-packets $\Pi_{\phi}$ of tempered representations $\pi \in \Pi_{\text {temp }}(G)$. It also represents a special case of our other family of parameters $\psi \in \Psi(G)$, which leads to packets $\Pi_{\psi}$ of unitary representations $\pi \in \Pi_{\text {unit }}(G)$, and will be the setting for the theorem we actually state. We do need to bear in mind that the packets $\Pi_{\psi}$ are larger than the $L$-packets $\Pi_{\phi_{\psi}}$ attached to the (unbounded) images $\phi_{\psi}$ (4.10) of $\psi$ in $\Phi(G)$. In particular, they are not in general a part of the local Langlands classification. Their role is rather to describe local constituents of automorphic representations.

We actually have to settle for something a little weaker. As we discussed at the end of the last section, the transfer mapping $\widetilde{f} \rightarrow \widetilde{f}^{G}$ from $\widetilde{\mathcal{H}}(N)$ to $\mathcal{S}(G)$ is not generally surjective. Its image is the subspace $\widetilde{\mathcal{S}}(G)$ of $\widetilde{O u t}_{N}(G)$-invariant functions in $\mathcal{S}(G)$. We have therefore to work with the set $\widetilde{\Psi}(G)$ of $\widetilde{\text {Outt}_{N}}(G)$-orbits in $\Psi(G)$. This fits into the sequence

$$
\widetilde{\Phi}_{\text {bdd }}(G) \subset \widetilde{\Psi}(G) \subset \widetilde{\Phi}(G)
$$

of families of orbits of parameters. The group $\widetilde{O u t}_{N}(G)$ (of order 1 or 2) acts by outer automorphisms also on $G(F)$, and hence on equivalence classes of irreducible representations. We therefore have an associated sequence

$$
\widetilde{\Pi}_{\text {temp }}(G) \subset \widetilde{\Pi}_{\text {unit }}(G) \subset \widetilde{\Pi}(G)
$$

of families of orbits of representations. This qualification is only relevant to the case that $G$ is type $\mathbf{D}_{n}$. If $G$ is of type $\mathbf{B}_{n}$ or $\mathbf{C}_{n}$, Out ${ }_{N}(G)$ is trivial, and the sets are unchanged from before.

We have thus to attach packets $\widetilde{\Pi}_{\psi}$ in $\widetilde{\Pi}_{\text {unit }}(G)$ to parameters $\psi \in \widetilde{\Psi}(G)$. We expect them to be multiplicity free. If $F$ is nonarchimedean, this is a deep theorem of Moeglin [M2]. If $F$ is archimedean, however, it is unknown. We have therefore to formulate the local theorem as an assertion for packets with multiplicities.

If $S$ is any set, a set over $S$, or an $S$-set, or even an $S$-packet will mean simply a set $S_{1}$ with a fibration

$$
S_{1} \longrightarrow S
$$

over $S$. Any function on $S$, such as the character $f_{G}(\pi)$ on $\widetilde{\mathcal{H}}(G)$ in case $S$ equals $\widetilde{\Pi}(G)$, will be identified with its pullback to a function on $S_{1}$. The order

$$
m_{1}: S \longrightarrow \mathbb{N} \cup\{0, \infty\}
$$

of the fibres in $S_{1}$ represents a multiplicity function, which makes $S$ into what can be called a multiset on $S$. If $S_{1}$ is multiplicity free, in that every element in $S$ has multiplicity at most 1 , it is of course just a subset of $S$. 
TheOREm 7.1. Assume that $F$ is local and that $G \in \widetilde{\mathcal{E}}(N)$.

(a) For any local parameter $\psi \in \widetilde{\Psi}(G)$, there is a finite packet $\widetilde{\Pi}_{\psi}$ over $\widetilde{\Pi}_{\text {unit }}(G)$, together with a mapping

$$
\pi \longrightarrow\langle\cdot, \pi\rangle, \quad \pi \in \widetilde{\Pi}_{\psi},
$$

from $\widetilde{\Pi}_{\psi}$ to the group $\widehat{\mathcal{S}}_{\psi}$ of irreducible characters on $\mathcal{S}_{\psi}$, with the following property: if $s$ is a semisimple element in the centralizer $S_{\psi}=S_{\psi}(G)$ and $\left(G^{\prime}, \psi^{\prime}\right)$ is the preimage of $(\psi, s)$ under the local version of the correspondence (5.11) in $\S 5$, then

$$
f^{\prime}\left(\psi^{\prime}\right)=\sum_{\pi \in \widetilde{\Pi}_{\psi}}\left\langle s_{\psi} x, \pi\right\rangle f_{G}(\pi), \quad f \in \widetilde{\mathcal{H}}(G),
$$

where $x$ is the image of $s$ in $\mathcal{S}_{\psi}$, and $s_{\psi}$ is the image in $\mathcal{S}_{\psi}$ of the element (5.6).

(b) If $\psi=\phi$ belongs to the subset $\widetilde{\Phi}_{\mathrm{bdd}}(G)$ of parameters in $\widetilde{\Psi}(G)$ that are trivial on the factor $S U(2)$, the elements in $\widetilde{\Pi}_{\phi}$ are tempered and multiplicity free, and the corresponding mapping from $\widetilde{\Pi}_{\phi}$ to $\widehat{\mathcal{S}}_{\phi}$ is injective. Moreover, every element in $\widetilde{\Pi}_{\text {temp }}(G)$ belongs to exactly one packet $\widetilde{\Pi}_{\phi}$. Finally, if $F$ is nonarchimedean, the mapping from $\widetilde{\Pi}_{\phi}$ to $\widehat{\mathcal{S}}_{\phi}$ is bijective.

Remarks. 1. The premise of this theorem depends on Theorem 6.1. To be precise, the left hand side of (7.2) is a product of linear forms (6.8) postulated by the earlier theorem, taken over the factors of the endoscopic group $G^{\prime}$. Composed with the transfer mapping $f \rightarrow f^{\prime}$, it represents a linear form on $\widetilde{\mathcal{H}}(G)$. As such, it determines the packet $\widetilde{\Pi}_{\psi}$ and the pairing $\langle x, \pi\rangle$ from the expression on the right hand side of (7.2).

2. The finite subsets $\widetilde{\Pi}_{\phi}$ of $\widetilde{\Pi}_{\text {temp }}(G)$ in (b) represent the tempered $L$-packets. They are composed of $\widetilde{O}_{N}(G)$-orbits of tempered representations, which are parametrized by characters in $\widehat{\mathcal{S}}_{\phi}$. Since the theorem posits a disjoint union

$$
\widetilde{\Pi}_{\text {temp }}(G)=\coprod_{\widetilde{\Phi}_{\text {bdd }}(G)} \widetilde{\Pi}_{\phi},
$$

it can be regarded as an endoscopic classification of the irreducible tempered representations of $G(F)$. It amounts to the local Langlands correspondence for $G$ if $G$ is of type $\mathbf{B}_{n}$ or $\mathbf{C}_{n}$, and something slightly weaker if $G$ is of type $\mathbf{D}_{n}$.

3. Suppose that $F$ is archimedean. Shelstad has established a general classification

$$
\Pi_{\mathrm{temp}}(G)=\coprod_{\Phi_{\mathrm{bdd}}(G)} \Pi_{\phi}
$$

of $\Pi_{\text {temp }}(G)$ for any real group $G$, in terms of $L$-packets $\Pi_{\phi}$ that satisfy endoscopic character relations (7.2) (with $s_{\psi}=1$ ). In the papers [She1]-[She4], she does not define the stable distributions on the left hand side of (7.2) (in case $G \in \widetilde{\mathcal{E}}_{\text {sim }}(N)$ and $\psi=\phi$ ) in terms of twisted transfer from general linear groups. However, this property (and more) is established in the recent papers of Shelstad [She5] and Mezo [Me]. We depend on these results for our proof of the theorem for nonarchimedean $F$ (and for the proof of the global theorems we will state presently). For general parameters $\psi$, Adams, Barbasch and Vogan $[\mathbf{A B V}]$ have constructed packets $\Pi_{\psi}$ that satisfy relations (7.2), again for any real group $G$. However, it is not presently known whether the stable distributions they define for the left hand 
side of (7.2) match those we obtain by twisted transfer from general linear groups (in our case that $G \in \widetilde{\mathcal{E}}_{\text {sim }}(N)$ ). The point is important for our purposes, because the global results at which the local packets $\widetilde{\Pi}_{\psi}$ are ultimately aimed are all proved by comparison with the twisted trace formula for $G L(N)$.

4. Suppose that $F$ is nonarchimedean. The structure of the general packets $\widetilde{\Pi}_{\psi}$ is better known than in the archimedean case, thanks to the work of Moeglin. We have already mentioned her proof [M2] that the packets $\widetilde{\Pi}_{\psi}$ are multiplicity free. This is a consequence of a general algorithm [M1] for computing the Langlands parameters of elements in $\widetilde{\Pi}_{\psi}$, assuming the classification of the tempered representations $\widetilde{\Pi}_{\text {temp }}(G)$ provided by part (b) of the theorem.

Suppose that $\psi$ belongs to the larger set $\widetilde{\Psi}^{+}(G)$ of local parameters for $G$, defined without the condition that their restriction to $L_{F}$ be bounded. Then $\psi$ can be expressed rather simply as a composition

$$
\psi=\xi_{M} \circ \psi_{M, \lambda}
$$

where $\psi_{M, \lambda}$ is a twist of a parameter $\psi_{M} \in \widetilde{\Psi}(M)$ for a Levi subgroup $M$ of $G$ and $\xi_{M}$ the embedding of ${ }^{L} M$ into ${ }^{L} G$ attached to a parabolic subgroup $P \in \mathcal{P}(M)$ of $G$ with Levi component $M$. The twisting element $\lambda$ lies in the open chamber defined by $P$ in a certain real vector space

$$
\mathfrak{a}_{M}^{*}=X(M)_{F} \otimes \mathbb{R} .
$$

It can be identified with either a homomorphism from $L_{F} \times S L(2, \mathbb{C})$ into the connected component of 1 in $Z(\widehat{M})^{\Gamma_{F}}$, or a real quasicharacter on the group $M(F)$. With the former interpretation, we observe that $S_{\psi}$ is contained in $\widehat{M}$, and hence that the mapping $x \rightarrow x_{M}$ of $\mathcal{S}_{\psi_{M}}$ into $\mathcal{S}_{\psi}$ is an isomorphism. With the latter interpretation, we define the packet of $\psi$ as a corresponding set (of orbits of) induced representations, which we denote hesitantly as

$$
\widetilde{\Pi}_{\psi}=\left\{\pi=\mathcal{I}_{P}\left(\pi_{M, \lambda}\right): \pi_{M} \in \widetilde{\Pi}_{\psi_{M}}\right\} .
$$

It is bijective with $\widetilde{\Pi}_{\psi_{M}}$, and comes with a pairing

$$
\langle x, \pi\rangle=\left\langle x_{M}, \pi_{M}\right\rangle, \quad x \in \mathcal{S}_{\psi}, \pi \in \widetilde{\Pi}_{\psi},
$$

with $\mathcal{S}_{\psi}$. The assertion (a) of Theorem 7.1 for the more general parameter $\psi$, with the understanding that the elements in the packet $\widetilde{\Pi}_{\psi}$ might now be reducible, then follows from its analogue for $\psi_{M}$, and the standard character formula for an induced representation.

Consider the case that the local parameter $\psi=\phi$ in $\widetilde{\Psi}^{+}(G)$ is trivial on the factor $S U(2)$. Then $\phi$ belongs to the set $\widetilde{\Phi}(G)$ of general (unbounded) Langlands parameters. It has a decomposition $\phi=\xi_{M} \circ \phi_{M, \lambda}$, as above. However, we shall denote the packet we introduced above differently, as

$$
\widetilde{P}_{\psi}=\left\{\rho=\mathcal{I}_{P}\left(\pi_{M, \lambda}\right): \pi_{M} \in \widetilde{\Pi}_{\phi_{M}}\right\} .
$$

(The $P$ in $\widetilde{P}_{\psi}$ is to be understood as an upper case $\rho$.) We reserve the symbol $\widetilde{\Pi}_{\psi}$ for the packet

$$
\widetilde{\Pi}_{\psi}=\left\{\pi=\pi_{\rho}: \rho \in \widetilde{P}_{\psi}\right\}
$$


of irreducible Langlands quotients of representations in $\widetilde{P}_{\psi}$. It comes with the pairing

$$
\langle x, \pi\rangle=\left\langle x, \pi_{\rho}\right\rangle=\langle x, \rho\rangle=\left\langle x_{M}, \pi_{M}\right\rangle, \quad x \in \mathcal{S}_{\psi}, \pi \in \widetilde{\Pi}_{\psi},
$$

with $\mathcal{S}_{\psi}$ that it inherits from $\widetilde{P}_{\psi}$. However, it does not satisfy the endoscopic character relation (7.2), since the Langlands quotient $\pi_{\rho}$ need not be induced. Nonetheless, the original Langlands classification [L5] for real groups (extended to $p$-adic groups in $[\mathbf{B W}]$ ) tells us that

$$
\widetilde{\Pi}(G)=\coprod_{\phi \in \widetilde{\Phi}(G)} \widetilde{\Pi}_{\phi}
$$

We therefore obtain an explicit classification of general representations $\pi \in \widetilde{\Pi}(G)$ from the tempered case given by Theorem 7.1(b).

Incidentally, it would appear that the notation in (7.5) is in conflict with that of (7.6). We hope that it will not be! For we shall consider only parameters $\psi \in$ $\widetilde{\Psi}^{+}(G)$ that arise from the local components of automorphic representations. That we have to take them in the larger set $\widetilde{\Psi}^{+}(G)$ is a necessary consequence of our not having at our disposal the generalized Ramanujan conjecture for $G L(N)$, as we have noted. But it is still a pretty stringent restriction, which we conjecture implies that the induced representations $\rho=\mathcal{I}_{P}\left(\pi_{M, \lambda}\right)$ are irreducible (and unitary) [ECR, Conjecture 8.3.1]. If this is so, each $\rho$ equals its Langlands quotient $\pi_{\rho}$, and there is no conflict with the notation (7.5).

Suppose now that the field $F$ is global. There will be two global theorems for the group $G \in \widetilde{\mathcal{E}}_{\text {sim }}(N)$ over $F$. The first is the central result. It gives a decomposition of the automorphic discrete spectrum of $G$ in terms of global parameters in the subset

$$
\widetilde{\Psi}_{2}(G)=\left\{\psi \in \widetilde{\Psi}(G):\left|S_{\psi}\right|<\infty\right\}
$$

of $\widetilde{\Psi}(G)$ and the local objects of Theorem 7.1(a). It is best formulated in terms of the global Hecke algebra $\mathcal{H}(G)$ of functions on $G(\mathbb{A})$, with respect to a suitable maximal compact subgroup

$$
K=\prod_{v} K_{v}
$$

of $G(\mathbb{A})$.

By definition, $\mathcal{H}(G)$ is the space of finite linear combinations of products

$$
\prod_{v} f_{v}, \quad f_{v} \in \mathcal{H}\left(G_{v}\right)
$$

such that $f_{v}$ is the characteristic function of $K_{v}$ for almost all $v$. We write $\widetilde{\mathcal{H}}(G)$ for the locally symmetric subalgebra, in which each $f_{v}$ lies in the subalgebra $\widetilde{\mathcal{H}}\left(G_{v}\right)$ of $\mathcal{H}\left(G_{v}\right)$. For any function $f$ in $\widetilde{\mathcal{H}}(G)$, and any admissible representation

$$
\pi=\bigotimes_{v} \pi_{v}, \quad \pi_{v} \in \Pi\left(G_{v}\right)
$$

the character $f_{G}(\pi)$ depends only on the Out ${ }_{N}\left(G_{v}\right)$-orbit of $\pi_{v}$ in $\widetilde{\Pi}\left(G_{v}\right)$, for any $v$. We will have to describe the discrete spectrum of $G$ as an $\widetilde{\mathcal{H}}(G)$-module, since the local packets consist of $\widetilde{O u t}_{N}\left(G_{v}\right)$-orbits $\pi_{v}$ in $\widetilde{\Pi}_{\text {unit }}\left(G_{v}\right)$. Of course if $G$ is of type $\mathbf{B}_{n}$ or $\mathbf{C}_{n}$, the groups $\operatorname{Out}_{N}\left(G_{v}\right)$ are all trivial, and $\widetilde{\mathcal{H}}(G)$ equals $\mathcal{H}(G)$. We would 
then have a description as an $\mathcal{H}(G)$-module, or for that matter, a decomposition in terms of irreducible representations $\pi \in \Pi_{\text {unit }}(G)$.

We are assuming the seed Theorems 5.1 and 5.2. The first of these was needed to define the global set $\widetilde{\Psi}(G)$ itself. The second led us to a localization mapping $\psi \rightarrow \psi_{v}$ from $\widetilde{\Psi}(G)$ to the local set $\widetilde{\Psi}_{v}^{+}(G)$. The local theorem we have just stated (together with the ensuing discussion) allows us to attach a local packet $\widetilde{\Pi}_{\psi_{v}}$ to $\psi$ and $v$. We can thus attach a global packet

$$
\widetilde{\Pi}_{\psi}=\left\{\pi=\bigotimes_{v} \pi_{v}: \pi_{v} \in \widetilde{\Pi}_{\psi_{v}},\left\langle\cdot, \pi_{v}\right\rangle=1 \text { for almost all } v\right\}
$$

of (orbits of) representations of $G(\mathbb{A})$ to any $\psi \in \widetilde{\Psi}(G)$. Any element $\pi$ in the global packet $\widetilde{\Pi}_{\psi}$ determines a character

$$
\langle x, \pi\rangle=\prod_{v}\left\langle x_{v}, \pi_{v}\right\rangle, \quad x \in \mathcal{S}_{\psi},
$$

on the global centralizer quotient $\mathcal{S}_{\psi}$. On the right hand side of (7.9), the product can be taken over a finite set, while $x \rightarrow x_{v}$ is the mapping from $\mathcal{S}_{\psi}$ to $\mathcal{S}_{\psi_{v}}$ we have discussed earlier.

TheOREM 7.2. Assume that $F$ is global and that $G \in \widetilde{\mathcal{E}}_{\text {sim }}(N)$. Then there is an $\tilde{\mathcal{H}}(G)$-module isomorphism

$$
L_{\text {disc }}^{2}(G(F) \backslash G(\mathbb{A})) \cong \bigoplus_{\psi \in \widetilde{\Psi}_{2}(G)} m_{\psi}\left(\bigoplus_{\pi \in \widetilde{\Pi}_{\psi}\left(\varepsilon_{\psi}\right)} \pi\right),
$$

where $m_{\psi}$ equals 1 or 2 and

$$
\varepsilon_{\psi}: \mathcal{S}_{\psi} \longrightarrow\{ \pm 1\}
$$

is a linear character defined explicitly in terms of symplectic $\varepsilon$-factors, while $\widetilde{\Pi}_{\psi}\left(\varepsilon_{\psi}\right)$ is the subset of representations $\pi$ in the global packet $\widetilde{\Pi}_{\psi}$ such that the character $\langle\cdot, \pi\rangle$ on $\mathcal{S}_{\psi}$ equals $\varepsilon_{\psi}$.

The statement will not be complete until we define the integer $m_{\psi}$ and the character $\varepsilon_{\psi}$. The first of these is quite elementary. The global parameter $\psi$ comes with the $L$-embedding

$$
\widetilde{\psi}_{G}: \mathcal{L}_{\psi} \times S L(2, \mathbb{C}) \longrightarrow{ }^{L} G
$$

determined as a $\widehat{G}$-orbit up to the action of the group $\widetilde{O u t}_{N}(G)$. We define $m_{\psi}$ for any $\psi \in \widetilde{\Psi}(G)$ to be the number of $\widehat{G}$-orbits in the associated $\widetilde{O} u_{N}(G)$-orbit. For an equivalent description, we write the parameter $\psi \in \widetilde{\Psi}_{2}(G)$ as

$$
\psi=\psi_{1} \boxplus \cdots \boxplus \psi_{r},
$$

for distinct, self dual factors $\psi_{i} \in \widetilde{\Psi}_{\operatorname{sim}}\left(N_{i}\right)$. One checks that $m_{\psi}$ equals 1 unless $G$ is of type $\mathbf{D}_{n}$ (or in other words, $N$ is even and $\widehat{G}=S O(N, \mathbb{C})$ ), and the rank $N_{i}$ of each of the components $\psi_{i}$ is also even, in which case $m_{\psi}$ equals 2 . The integer $m_{\psi}$ obviously bears on the question of the multiplicity with which a representation $\pi$ occurs in the automorphic discrete spectrum, but one also needs information about the localizations $\psi_{v}$. For a full statement in the case that $\psi=\phi$ lies in the subset $\widetilde{\Phi}_{\text {bdd }}(G)$ of $\widetilde{\Psi}(G)$, see $[\mathbf{A} 4, \S 3($ vii $)]$. 
The sign character $\varepsilon_{\psi}$ is more interesting. We first make an observation on some general $\varepsilon$-factors. Suppose that $\psi \in \widetilde{\Psi}(N)$ is an arbitrary global parameter, and that $r$ is an arbitrary finite dimensional representation of $\mathcal{L}_{\psi}$, subject only to the condition that its equivalence class is stable under the group $\operatorname{Aut}\left(\mathcal{L}_{\psi}\right)$. Then $r$ pulls back to a well defined representation $r_{v}$ of $L_{F_{v}}$, for any $v$. We can therefore define the global $L$-function

$$
L(s, r)=\prod_{v} L\left(s, r_{v}\right)
$$

by an Euler product that converges for the real part of $s$ large. We do not know that it has analytic continuation and functional equation, since it really amounts to a rather general automorphic $L$-function, even though its local factors are arithmetic $L$-functions defined as in $[\mathbf{T}]$. But we can still define the corresponding global $\varepsilon$ factor as a finite product

$$
\varepsilon\left(s, r, \psi_{F}\right)=\prod_{v} \varepsilon\left(s, r_{v}, \psi_{F_{v}}\right) .
$$

Again, we cannot say that this function is independent of the nontrivial additive character $\psi_{F}$ on $\mathbb{A} / F$. But if $r$ is symplectic, by which we mean that it takes values in the symplectic subgroup of the underlying general linear group, the value of the local factor $\varepsilon\left(s, r_{v}, \psi_{F_{v}}\right)$ at $s=\frac{1}{2}$ is known to equal +1 or -1 , and to be independent of $\psi_{F_{v}}$. We therefore have a global sign

$$
\varepsilon\left(\frac{1}{2}, r\right)=\varepsilon\left(\frac{1}{2}, r, \psi_{F}\right)= \pm 1
$$

in this case, which is independent of $\psi_{F}$.

We can define $\varepsilon_{\psi}$ if $\psi$ is a general parameter in $\widetilde{\Psi}(G)$. We first define a representation

$$
\tau_{\psi}: S_{\psi} \times \mathcal{L}_{\psi} \times S L(2, \mathbb{C}) \longrightarrow G L(\widehat{\mathfrak{g}})
$$

on the Lie algebra of $\widehat{\mathfrak{g}}$ of $\widehat{G}$ by setting

$$
\tau_{\psi}(s, g, h)=\operatorname{Ad}_{G}\left(s \cdot \widetilde{\psi}_{G}(g, h)\right), \quad s \in S_{\psi},(g, h) \in \mathcal{L}_{\psi} \times S L(2, \mathbb{C}),
$$

where $\operatorname{Ad}_{G}$ is the adjoint representation of ${ }^{L} G$. This representation is orthogonal, and hence self-dual, since it is invariant under the Killing form on $\widehat{\mathfrak{g}}$. Let

$$
\tau_{\psi}=\bigoplus_{\alpha} \tau_{\alpha}=\bigoplus_{\alpha}\left(\lambda_{\alpha} \otimes \mu_{\alpha} \otimes \nu_{\alpha}\right)
$$

be its decomposition into irreducible representations $\lambda_{\alpha}, \mu_{\alpha}$ and $\nu_{\alpha}$ of the respective groups $S_{\psi}, \mathcal{L}_{\psi}$ and $S L(2, \mathbb{C})$. We then define

$$
\varepsilon_{\psi}(x)=\prod_{\alpha}^{\prime} \operatorname{det}\left(\lambda_{\alpha}(s)\right), \quad s \in S_{\psi},
$$

where $x$ is the image of $s$ in $\mathcal{S}_{\psi}$, and $\prod^{\prime}$ denotes the product over those indices $\alpha$ with $\mu_{\alpha}$ symplectic and with

$$
\varepsilon\left(\frac{1}{2}, \mu_{\alpha}\right)=-1 \text {. }
$$

The second global theorem is a supplement to the first. It gives a long conjectured $L$-function criterion for whether a self-dual cuspidal automorphic representation of $G L(N)$ is symplectic or orthogonal, in the sense that it is a functorial image from a group $G$ whose $L$-group is symplectic or orthogonal. It also gives an automorphic analogue of a well known property [FQ], [D] of orthogonal (arithmetic) root numbers. The main point for us, however, is that this theorem has a critical 
role in the proofs of all of the theorems. It is an indispensable part of what governs the signs that arise in the comparison of trace formulas.

To place the second global theorem in context, we observe a property of certain Rankin-Selberg $L$-functions. The Rankin-Selberg representation of $G L(N, \mathbb{C}) \times$ $G L(N, \mathbb{C})$ on the space of complex $(N \times N)$-matrices is irreducible, but its restriction to the diagonal image of $G L(N, \mathbb{C})$ is a direct sum $S^{2} \oplus \Lambda^{2}$, where $S^{2}$ (resp. $\Lambda^{2}$ ) is the representation of $G L(N, \mathbb{C})$ on the space of symmetric (resp. skew-symmetric) $(N \times N)$-matrices. If $\pi$ is a cuspidal automorphic representation of $G L(N)$, the diagonal Rankin-Selberg functions then break into formal products

$$
L(s, \pi \times \pi)=L\left(s, \pi, S^{2}\right) L\left(s, \pi, \Lambda^{2}\right)
$$

and

$$
\varepsilon(s, \pi \times \pi)=\varepsilon\left(s, \pi, S^{2}, \psi_{F}\right) \varepsilon\left(s, \pi, \Lambda^{2}, \psi_{F}\right) .
$$

The two $L$-functions on the right hand side of (7.11) are among the cases of the Langlands-Shahidi method treated in [Sha]. In both cases, the local $L$-functions and $\varepsilon$-factors can be constructed, with the consequence that the formal products (7.11) and (7.12) become actual products, for which the resulting two global $L$-functions have analytic continuation with functional equation (4.15).

Suppose that $\phi \in \widetilde{\Phi}_{\operatorname{sim}}(N)$ is a simple generic parameter. For us, this amounts to a cuspidal automorphic representation $\pi=\pi_{\phi}$ of $G L(N)$, which is now self-dual. Theorem 5.1 asserts that $\phi$ belongs to the subset

$$
\widetilde{\Phi}_{\text {sim }}(G)=\widetilde{\Phi}_{\operatorname{sim}}(N) \cap \widetilde{\Psi}(G),
$$

for a unique $G \in \mathcal{E}_{\text {sim }}(N)$. We need to understand how $G$ is related to $L$-functions and $\varepsilon$-factors. It is known that the Rankin-Selberg $L$-function

$$
L(s, \phi \times \phi)=L(s, \pi \times \pi)=L\left(s, \pi \times \pi^{\vee}\right)
$$

has a pole of order 1 at $s=1$. It is also known that neither of the corresponding factors $L\left(s, \phi, S^{2}\right)$ and $L\left(s, \phi, \Lambda^{2}\right)$ on the right hand side of (7.12) has a zero at $s=1$. It follows that exactly one of them has a pole at $s=1$ (which will be of order 1). This motivates the assertion (a) of our second global theorem.

Theorem 7.3. Assume that $F$ is global.

(a) Suppose that $G \in \widetilde{\mathcal{E}}_{\text {sim }}(N)$ and that $\phi$ belongs to $\widetilde{\Phi}_{\text {sim }}(G)$. Then $\widehat{G}$ is orthogonal if and only if the symmetric square L-function $L\left(s, \phi, S^{2}\right)$ has a pole at $s=1$, while $\widehat{G}$ is symplectic if and only if the skew-symmetric square L-function $L\left(s, \phi, \Lambda^{2}\right)$ has a pole at $s=1$.

(b) Suppose that for $i=1,2, \phi_{i}$ belongs to $\widetilde{\Phi}_{\text {sim }}\left(G_{i}\right)$, for simple endoscopic data $G_{i} \in \widetilde{\mathcal{E}}_{\text {sim }}\left(N_{i}\right)$. Then the associated Rankin-Selberg $\varepsilon$-factor satisfies

$$
\varepsilon\left(\frac{1}{2}, \phi_{1} \times \phi_{2}\right)=1,
$$

if $\widehat{G}_{1}$ and $\widehat{G}_{2}$ are either both orthogonal or both symplectic.

We have completed the formal statements of the three main theorems of $[\mathbf{E C R}]$. Representations of quasisplit special orthogonal and symplectic groups $G$ have been studied from other points of view. There is a rather complete theory for the special case of representations with Whittaker models [CKPS], [GRS]. Since Theorems 7.1 and 7.2 give a classification of local and global representations, it is reasonable to ask which of these have Whittaker models. The answer is given in [ECR, §8.3], following 
a conjecture in [Sha]. The $\theta$-correspondence has been a different source of results. (See $[\mathbf{K u}]$, for example.) It would be very interesting to compare the relations it has provided with the classification of Theorems 7.1 and 7.2. Considerably more is known in the local case, thanks to the article [M3] and its predecessors. For global $F$, we refer the reader to $[\mathbf{J i}]$ for examples and a description of some of the problems.

Some applications of the theorems were listed in $[\mathbf{A 4}, \S 3]$. There will no doubt be others. Some will follow from the extension of the theorems to orthogonal and symplectic groups that are not quasisplit. For a description of a proposed classification of representations for such groups, which remains conjectural, see [ECR, §9]. Other applications await an extension of the theorems to different groups, such as the split group $G S p(2 n)$ of symplectic similitudes, for example.

\section{Implications for functoriality}

It remains to add some observations on functoriality (Principle 1.1). We consider the two cases

$$
\left(G, G^{\prime}, \rho\right)=(G L(N), G, \xi), \quad G \in \widetilde{\mathcal{E}}_{\mathrm{sim}}(N),
$$

and

$$
\left(G, G^{\prime}, \rho\right)=\left(G, G^{\prime}, \xi^{\prime}\right), \quad G \in \widetilde{\mathcal{E}}_{\mathrm{sim}}(N), G^{\prime} \in \mathcal{E}_{\text {ell }}(G),
$$

discussed at the end of $\S 1$. What are the implications of the theorems we have stated?

We first observe that in both cases, we have a mapping

$$
\psi^{\prime} \longrightarrow \psi=\rho \circ \psi^{\prime}=\rho\left(\psi^{\prime}\right), \quad \psi^{\prime} \in \widetilde{\Psi}\left(G^{\prime}\right),
$$

from $\widetilde{\Psi}\left(G^{\prime}\right)$ to $\widetilde{\Psi}(G)$. This is obvious if $F$ is local, and would be so in the global case as well if our parameters were defined on the Langlands group $L_{F}$. As matters stand, we must appeal to the definitions of $\S 5$ for global $F$, which depend on the nontrivial Theorems 5.1 and 5.2. In any case, we do have the mapping (8.3) for any $F$. As usual, we have particular interest in its restriction to the subset $\widetilde{\Phi}_{\text {bdd }}\left(G^{\prime}\right)$ of $\widetilde{\Psi}\left(G^{\prime}\right)$.

Suppose for the moment that $F$ is local. Local functoriality in the first case (8.1) is given by Theorem 6.1 and the definition (6.7) of the linear form on the right hand side of (6.9). In the second case, it is given by the character identity (7.2) in Theorem 7.1(a) and the bijective correspondence (5.11). Because these two cases have their origins in the theory of endoscopy, we obtain an explicit description of the local functoriality correspondence. It is given by the relation

$$
\pi^{\prime} \longrightarrow \pi, \quad \pi^{\prime} \in \widetilde{\Pi}\left(G^{\prime}\right),
$$

where for a given $\pi^{\prime}, \pi$ ranges over the representations in the packet $\widetilde{\Pi}_{\phi}$ of the local parameter $\phi \in \widetilde{\Phi}(G)$ such that $\phi=\rho\left(\phi^{\prime}\right)$ is the image of the parameter $\phi^{\prime} \in \widetilde{\Phi}\left(G^{\prime}\right)$ with $\pi^{\prime} \in \widetilde{\Pi}_{\phi^{\prime}}$. In other words, it is the correspondence

$$
\widetilde{\Pi}_{\phi^{\prime}} \longrightarrow \widetilde{\Pi}_{\phi}
$$

in which $\phi^{\prime}$ ranges over the local parameters in $\widetilde{\Phi}\left(G^{\prime}\right)$ and $\phi^{\prime} \rightarrow \phi$ is the mapping from $\widetilde{\Phi}\left(G^{\prime}\right)$ to $\widetilde{\Phi}(G)$ analogous to $(8.3)$. 
Functoriality is called a "principle" for a reason. It represents a phenomenon, which we can almost regard as a general law of nature, rather than a precise conjecture. It is capable of taking different forms, which depend on the context. This flexibility is built into its statement in $\S 1$, by postulating a correspondence rather than a mapping, whose precise nature is left unspecified. For example, in the local case above, we can consider packets $\widetilde{P}_{\phi}$ of induced representations $\rho$ (sometimes known as standard representations) in place of packets $\widetilde{\Pi}_{\phi}$ of irreducible Langlands quotients $\pi$. Let $\widetilde{\Pi}_{\phi}^{+}$be the set of irreducible constituents $\pi$ of these representations, a packet that contains $\widetilde{\Pi}_{\phi}$, and equals $\widetilde{\Pi}_{\phi}$ if $\phi$ lies in the subset $\widetilde{\Phi}_{\text {bdd }}(G)$ of $\widetilde{\Phi}(G)$. These larger packets are no longer disjoint, in contrast to the $L$-packets $\widetilde{\Pi}_{\phi}$. This leads to a more complicated version of local functoriality. We take it to be the coarser relation

$$
\pi^{\prime} \longrightarrow \pi, \quad \pi^{\prime} \in \widetilde{\Pi}\left(G^{\prime}\right),
$$

where for a given $\pi^{\prime}, \pi$ ranges over the representations in packets $\widetilde{\Pi}_{\phi}^{+}$of local parameters $\phi \in \widetilde{\Phi}^{+}(G)$ such that $\phi=\rho\left(\phi^{\prime}\right)$ is the image as in (8.3) of a parameter $\phi^{\prime} \in \widetilde{\Phi}\left(G^{\prime}\right)$ with $\pi^{\prime} \in \widetilde{\Pi}_{\phi^{\prime}}^{+}$. We should also not be distracted by the fact that $\widetilde{P}_{\phi}$ and $\widetilde{\Pi}_{\phi}$ consist of orbits of irreducible representations (unless $G=G L(N)$ ), rather than actual representations. This is a side issue, which is not present if $G$ is of type $\mathbf{B}_{n}$ or $\mathbf{C}_{n}$, and is not particularly significant for what we are discussing here.

Assume now that $F$ is global. Global functoriality is harder to describe. For among other things, the statement of Principle 1.1 is predicated on the broader, formal definition $[\mathbf{L 3}]$ of automorphic representations, which we have not given. We can, however, formulate it without difficulty for the subsets

$$
\widetilde{\Pi}_{\phi, \text { aut }} \subset \widetilde{\Pi}_{\phi}, \quad \phi \in \widetilde{\Phi}_{\text {bdd }}(G),
$$

of automorphic representations in the global packets attached to parameters in $\widetilde{\Phi}_{\text {bdd }}(G)$. In this case, global functoriality is given by the correspondence

$$
\widetilde{\Pi}_{\phi^{\prime}, \text { aut }} \longrightarrow \widetilde{\Pi}_{\phi, \text { aut }}
$$

in which $\phi^{\prime}$ ranges over global parameters in $\widetilde{\Phi}_{\mathrm{bdd}}\left(G^{\prime}\right)$, and $\phi^{\prime} \rightarrow \phi$ is the mapping given by the restriction of (8.3) to the subset $\widetilde{\Phi}_{\text {bdd }}\left(G^{\prime}\right)$ of $\widetilde{\Psi}\left(G^{\prime}\right)$. The correspondence follows from the definitions of $\S 5$, the multiplicity formula of Theorem 7.2, and the reduction by Eisenstein series of the full automorphic spectrum to relatively discrete automorphic spectra for Levi subgroups. This reduction also gives an explicit characterization of the subset $\widetilde{\Pi}_{\phi, \text { aut }}$ of $\widetilde{\Pi}_{\phi}$.

More generally, consider a global parameter $\phi$ in the larger set $\widetilde{\Phi}(G)$. We can then form the global packet

$$
\widetilde{\Pi}_{\phi}^{+}=\left\{\pi=\widetilde{\bigotimes}_{v} \pi_{v}: \pi_{v} \in \widetilde{\Pi}_{\phi_{v}}^{+}, \pi_{v} \text { unramified for almost all } v\right\},
$$

in analogy with (2.2). The global parameter $\phi$ can be represented as the image of a "discrete" parameter $\phi_{M} \in \widetilde{\Phi}_{2}(M)$, for a Levi subgroup $M$ of $G$, under the dual embedding ${ }^{L} M \subset{ }^{L} G$. Let $\widetilde{\Pi}_{\phi \text {,aut }}^{+}$be the subset of irreducible representations in $\widetilde{\Pi}_{\phi}^{+}$obtained from parabolic induction from representations in the subset $\widetilde{\Pi}_{\phi_{M} \text {, aut }}$ of $\widetilde{\Pi}_{\phi_{M}}$. It is then a consequence of the definitions [L3, p. 203] that $\widetilde{\Pi}_{\phi \text {,aut }}^{+}$is the 
subset of representations in $\widetilde{\Pi}_{\phi}^{+}$that are automorphic in the extended sense of $[\mathbf{L 3}]$. In this more general setting, global functoriality is given by the correspondence

$$
\widetilde{\Pi}_{\phi^{\prime}, \text { aut }}^{+} \longrightarrow \widetilde{\Pi}_{\phi, \text { aut }}^{+},
$$

where $\phi^{\prime}$ ranges over global parameters in the larger set $\widetilde{\Phi}\left(G^{\prime}\right)$, and $\phi^{\prime} \rightarrow \phi$ is the mapping from $\widetilde{\Phi}\left(G^{\prime}\right)$ to $\widetilde{\Phi}(G)$ analogous to (8.3). It is clearly compatible with the local functoriality correspondence (8.5).

The global functoriality correspondence (8.6) treats many automorphic representations (in the extended sense of $[\mathbf{L 3}]$ ), but it still represents a special case. For example, it does not include the subset $\widetilde{\Pi}_{\psi \text {,cusp }}$ of cuspidal automorphic representations in a packet $\widetilde{\Pi}_{\psi}$, if $\psi$ lies in the complement of $\widetilde{\Phi}_{\text {bdd }}(G)$. This subset is a subtle object, which has been studied in depth by Moeglin. Leaving aside the question of how to characterize it explicity, we let $\widetilde{\Psi}^{+}(G)$ be the global analogue of the local set defined after the statement of Theorem 7.1. This will be the largest of our global sets of parameters, a family that contains both $\widetilde{\Psi}(G)$ and $\widetilde{\Phi}(G)$. As in the special case of the subset $\widetilde{\Phi}(G)$, a parameter $\psi$ in $\widetilde{\Psi}^{+}(G)$ is the image of a discrete parameter $\psi_{M} \in \widetilde{\Psi}_{2}^{+}(M)$, for some $M$. We then write $\widetilde{\Pi}_{\psi \text {,ic }}^{+}=\widetilde{\Pi}_{\psi \text {,ind-cusp }}^{+}$ for the packet of irreducible constituents of standard representations $\rho$ obtained by parabolic induction from representations in the subset $\widetilde{\Pi}_{\psi_{M} \text {,cusp }}$ of $\widetilde{\Pi}_{\psi_{M}}$. It lies in a chain

$$
\widetilde{\Pi}_{\psi, \text { ic }}^{+} \subset \widetilde{\Pi}_{\psi, \text { aut }}^{+} \subset \widetilde{\Pi}_{\psi}^{+},
$$

for packets $\widetilde{\Pi}_{\psi \text {,aut }}^{+}$and $\widetilde{\Pi}_{\psi}^{+}$defined in the same way, but with $\widetilde{\Pi}_{\psi_{M} \text {, cusp replaced }}$ by the larger sets $\widetilde{\Pi}_{\psi_{M} \text {,aut }}$ and $\widetilde{\Pi}_{\psi_{M}}$. The packet $\widetilde{\Pi}_{\psi}^{+}$, for example, is also equal to the set of restricted tensor products of representations in the local packets $\widetilde{\Pi}_{\psi_{v}}^{+}$, as in the special case of the global packet $\widetilde{\Pi}_{\phi}^{+}$defined above. As $\psi$ varies, the global packets $\widetilde{\Pi}_{\psi, \text { ic }}^{+}$are presumably disjoint, which would give a classification of the set $\widetilde{\Pi}_{\text {aut }}(G)$ below as a disjoint union of these packets.

General global functoriality ought then to be given by the generalization

$$
\widetilde{\Pi}_{\psi^{\prime}, \text { ic }}^{+} \longrightarrow \widetilde{\Pi}_{\psi, \text { ic }}^{+}, \quad \psi^{\prime} \in \widetilde{\Psi}^{+}\left(G^{\prime}\right),
$$

of (8.6). It would be compatible with a version of local functoriality that generalizes both the coarser relations (8.5) above and (8.11) below. I have not thought carefully about the implications of these constructions. Rather than pursue them further here, let me describe a simpler variant of global functoriality, which is easy to formulate.

We define

$$
\mathcal{C}_{\text {aut }}(G)=\left\{c(\pi): \pi \in \Pi_{\text {aut }}(G)\right\},
$$

where $\Pi_{\text {aut }}(G)$ is the set of representations

$$
\pi=\widetilde{\bigotimes}_{v} \pi_{v}
$$


of $G(\mathbb{A})$ that are automorphic in the sense of $[\mathbf{L 3}]$. Repeating what was implicit above, we write $\widetilde{\Pi}_{\text {aut }}(G)$ for the set of orbits of representations of $G(\mathbb{A})$ under the group

$$
\widetilde{\mathrm{Out}}_{N}\left(G_{\mathbb{A}}\right)=\prod_{v} \widetilde{\mathrm{Out}}_{N}\left(G_{v}\right)
$$

that have a representative in $\Pi_{\text {aut }}(G)$, for any group $G \in \widetilde{\mathcal{E}}_{\text {sim }}(N)$. If $G=G L(N)$, we take $\widetilde{\Pi}_{\text {aut }}(G)$ to be simply the set of self-dual representations in $\Pi_{\text {aut }}(G)$. In either case, we can then define a set

$$
\widetilde{\mathcal{C}}_{\text {aut }}(G)=\left\{c(\pi): \pi \in \widetilde{\Pi}_{\text {aut }}(G)\right\} .
$$

It consists of $\widetilde{O u t}_{N}\left(G_{\mathbb{A}}\right)$-orbits of (equivalence classes of) families of semisimple conjugacy classes with representatives in $\mathcal{C}_{\text {aut }}(G)$ if $G \in \widetilde{\mathcal{E}}_{\text {sim }}(N)$, and simply the subset of self-dual elements in $\mathcal{C}_{\text {aut }}(N)$ if $G=G L(N)$. We then have a mapping

$$
c^{\prime} \sim\left\{c_{v}^{\prime}: v \notin S^{\prime}\right\} \longrightarrow c=\rho\left(c^{\prime}\right) \sim\left\{\rho_{v}\left(c_{v}^{\prime}\right): v \notin S\right\}
$$

from $\widetilde{\mathcal{C}}_{\text {aut }}\left(G^{\prime}\right)$ to a larger set $\widetilde{\mathcal{C}}_{\mathbb{A}}(G)$, defined as above but without the condition of automorphy. We claim that this mapping takes $\widetilde{\mathcal{C}}_{\text {aut }}\left(G^{\prime}\right)$ to the subset $\widetilde{\mathcal{C}}_{\text {aut }}(G)$ of $\widetilde{\mathcal{C}}_{\mathbb{A}}(G)$. In other words, the image $\rho\left(c^{\prime}\right)$ of $c^{\prime}$ can be represented by an element $c$ in $\mathcal{C}_{\text {aut }}(G)$. This version of functoriality is less delicate than the others. We leave the reader to check that it follows from the various definitions and theorems above.

The mapping $c^{\prime} \rightarrow c$ from $\widetilde{\mathcal{C}}_{\text {aut }}\left(G^{\prime}\right)$ to $\widetilde{\mathcal{C}}_{\text {aut }}(G)$ is obviously quite concrete, like the sets of Hecke eigenfamilies that comprise its domain and codomain. It also illustrates the term "functoriality". For we can build a category $C$ from the groups we have been working with. Its objects are quasisplit groups over $F$, primarily the groups $G \in \widetilde{\mathcal{E}}_{\text {sim }}(N)$ and the general linear groups $G L(N)$, but also direct products of such groups. The morphisms hom $\left(G^{\prime}, G\right)$ between objects $G^{\prime}, G \in \operatorname{ob}(C)$ are $L$-homorphisms

$$
\rho:{ }^{L} G^{\prime} \longrightarrow{ }^{L} G
$$

given by (8.1) and (8.2), and whatever supplements are required for direct products. We then have a mapping

$$
F: G \longrightarrow \widetilde{\mathcal{C}}_{\text {aut }}(G), \quad G \in \mathrm{ob}(C),
$$

from objects $G \in \mathrm{ob}(C)$ to sets. The version of functoriality we have just formulated asserts that for any $\rho \in \operatorname{hom}\left(G^{\prime}, G\right)$, the mapping

$$
F(\rho): c^{\prime} \longrightarrow \rho\left(c^{\prime}\right), \quad c^{\prime} \in \widetilde{\mathcal{C}}_{\text {aut }}\left(G^{\prime}\right),
$$

given by $(8.9)$ takes the set $F\left(G^{\prime}\right)=\widetilde{\mathcal{C}}_{\text {aut }}\left(G^{\prime}\right)$ to the set $F(G)=\widetilde{\mathcal{C}}_{\text {aut }}(G)$. In other words, the mapping

$$
F: \mathrm{ob}(C) \longrightarrow \mathrm{ob}(S t)
$$

comes also with a mapping

$$
F: \operatorname{hom}\left(G^{\prime}, G\right) \longrightarrow \operatorname{hom}\left(F\left(G^{\prime}\right), F(G)\right), \quad G^{\prime}, G \in \mathrm{ob}(C),
$$

and is therefore a functor from $C$ to the category $S t$ of sets.

In this section, we have abandoned our informal characterization of automorphic representations, which we adopted for expository reasons in $\S 1$, for the broader definition in [L3]. We shall call the representations in this smaller class globally tempered automorphic representations, since we defined them in terms of global 
harmonic analysis. We have already agreed to denote them by $\Pi(G)$, for any one of our groups $G$ over the global field $F$. We thus have an embedding

$$
\Pi(G) \subset \Pi^{+}(G)
$$

where $\Pi^{+}(G)=\Pi_{\text {aut }}(G)$ is the set of general automorphic representations. This is parallel to the associated embedding

$$
\Psi(G) \subset \Psi^{+}(G)
$$

of global parameter sets. How would we formulate the principle of functoriality for globally tempered automorphic representations?

Given $G$, we have the associated sets $\widetilde{\Pi}(G) \subset \widetilde{\Pi}^{+}(G)$, which are parallel to the global parameter sets $\widetilde{\Psi}(G) \subset \widetilde{\Psi}^{+}(G)$. For any $\psi \in \Psi(G)$, we also have the subset

$$
\widetilde{\Pi}_{\psi, \text { aut }}=\widetilde{\Pi}_{\psi} \cap \widetilde{\Pi}(G)=\widetilde{\Pi}_{\psi} \cap \widetilde{\Pi}^{+}(G)
$$

of automorphic representations in the packet $\widetilde{\Pi}_{\psi}$. Functoriality for globally tempered automorphic representations will then be the correspondence

$$
\widetilde{\Pi}_{\psi^{\prime}, \text { aut }} \longrightarrow \widetilde{\Pi}_{\psi, \text { aut }},
$$

where $\psi^{\prime}$ ranges over the global parameters in $\widetilde{\Psi}\left(G^{\prime}\right)$, and $\psi^{\prime} \rightarrow \psi$ is the mapping (8.3) from $\widetilde{\Psi}\left(G^{\prime}\right)$ to $\widetilde{\Psi}(G)$.

The local analogue of (8.10) will have to be a little different from (8.4) and (8.5). Taking $F$ now to be local, we assume for simplicity that Conjecture 8.3.1 of $[\mathbf{E C R}]$ is valid (as we already have implicitly, in the notation of (8.10)). This was the assertion we mentioned in $\S 7$ that the local packets (7.5) and (7.6) are the same for the local parameters $\psi \in \widetilde{\Psi}^{+}(G)$ obtained from (globally tempered) automorphic representations. The conjecture was actually stated more generally for any $\psi$ in the intermediate set

$$
\widetilde{\Psi}(G) \subset \widetilde{\Psi}_{\text {unit }}^{+}(G) \subset \widetilde{\Psi}^{+}(G),
$$

defined in terms of unitary representations for $G L(N)$, following the statement of Theorem 1.5.1 of $[\mathbf{E C R}]$. Now as $\psi$ varies over even the smallest set $\widetilde{\Psi}(G)$, the associated packets $\widetilde{\Pi}_{\psi}$ are not disjoint, in contrast to the packets $\widetilde{\Pi}_{\phi}$ attached to parameters $\phi \in \widetilde{\Phi}(G)$. This complicates the local functoriality analogue of (8.10). We define it as the coarser relation

$$
\pi^{\prime} \longrightarrow \pi, \quad \pi^{\prime} \in \widetilde{\Pi}\left(G^{\prime}\right),
$$

where for a given $\pi^{\prime}, \pi$ ranges over the representations in packets $\widetilde{\Pi}_{\psi}$ of local parameters $\psi \in \widetilde{\Psi}_{\text {unit }}^{+}(G)$ such that $\psi=\rho\left(\psi^{\prime}\right)$ is the image (8.3) of a parameter $\psi^{\prime} \in \widetilde{\Psi}_{\text {unit }}^{+}\left(G^{\prime}\right)$ with $\pi^{\prime} \in \widetilde{\Pi}_{\psi^{\prime}}$. The (globally tempered) functoriality correspondence (8.10) is then compatible with its local analogue (8.11).

There is one last point, which might be somewhat surprising. It is conceivable that for global $F$, there could be elements $\psi \in \widetilde{\Psi}_{2}(G)$ such that the set $\widetilde{\Pi}_{\psi \text {, aut }}$ in (8.10) is empty. One sees easily that $\psi$ cannot contribute to the continuous automorphic spectrum of $G[\mathbf{A 5}, \S 3]$, so $\widetilde{\Pi}_{\psi \text {,aut }}$ is equal to the set $\widetilde{\Pi}_{\psi}\left(\varepsilon_{\psi}\right)$ of Theorem 7.2. Whether it is empty or not therefore depends on the sign character $\varepsilon_{\psi}$. Examples of this phenomenon were found some years ago by Cogdell and Piatetski-Shapiro $[\mathbf{C P}]$, by different methods. The question for us here is whether there is a global parameter $\psi^{\prime} \in \widetilde{\Psi}\left(G^{\prime}\right)$ with image $\psi=\rho\left(\psi^{\prime}\right)$ in $\widetilde{\Psi}(G)$ such that the set $\widetilde{\Pi}_{\psi \text {, aut }}$ is 
empty, but $\widetilde{\Pi}_{\psi^{\prime} \text {,aut }}$ is not. If the answer is affirmative, there will be a globally tempered automorphic representation $\pi^{\prime}$ of $G^{\prime}$ that is not in the domain of the global functoriality correspondence for $\rho$.

This last section has been written quite quickly. I hope that the discussion has not been too murky, and that it is essentially correct. It does seem to raise some interesting questions, which bear further reflection.

\section{References}

[ECR] J. Arthur, The Endoscopic Classification of Representations: Orthogonal and Symplectic Groups, Colloquium Publications, 61, 2013, American Mathematical Society.

[ABV] J. Adams, D. Barbasch, and D. Vogan, The Langlands Classification and Irreducible Characters for Real Reductive Groups, Progr. Math. 104, Birkhauser, Boston, 1992.

[A1] J. Arthur, A stable trace formula III. Proof of the main theorems, Annals of Math. 158 (2003), 769-873.

[A2] - A note on the automorphic Langlands group, Canad. Math. Bull. 45 (2002), $466-482$.

[A3] - An introduction to the trace formula, in Harmonic Analysis, the Trace Formula, and Shimura Varieties, Clay Mathematics Proceedings, vol. 4, 2005, 1-263.

[A4] - The Endoscopic Classification of Representations, in Automorphic Representations and L-functions, Tata Institute of Fundamental Research, 2013, 1-22.

[A5] - Eigenfamilies, characters and multiplicities, preprint.

[BJ] A. Borel and H. Jacquet, Automorphic forms and automorphic representations, in Automorphic Forms, Representations and L-functions, Proc. Symp. Pure Math. vol. 33, Part 1, Amer. Math. Soc., 1979, 189-202.

[BW] A. Borel and N. Wallach, Continuous Cohomology, Discrete Subgroups, and Representations of Reductive Groups, Ann. of Math. Studies 94, Princeton Univ. Press, Princeton, N.J., 1980.

[CKPS] J. Cogdell, H. Kim, I. Piatetski-Shapiro, and F. Shahidi, Functoriality for the classical groups, Publ. Math. Inst. Hautes Études Sci. 99 (2004), 163-233.

[CP] J. Cogdell and I. Piatetski-Shapiro, On base change for odd orthogonal groups, J. Amer. Math. 8 (1995), 975-996.

[D] P. Deligne, Les constantes locales de l'équation fonctionnelle de la fonction L d'Artin d'une représentation orthogonale, Invent. Math. 35 (1976), 299-316.

[F] D. Flath, Decomposition of representations into tensor products, in Automorphic Forms, Representations and L-functions, Proc. Sympos. Pure Math. vol. 33, Part 1, Amer. Math. Soc., 1979, 179-184.

[FLN] E. Frenkel, R. Langlands and B.C. Ngo, Formule des traces et functorialité: Le début d'un programme, preprint.

[FQ] A. Frohlich and J. Queyrot, On the functional equations of the Artin L-function for characters of real representations, Invent. Math. 20 (1973), 125-138.

[GRS] D. Ginzburg, S. Rallis, and D. Soudry, The descent map from automorphic representations of $G L(n)$ to classical groups, World Scientific Publishing Co. Pte. Ltd., Hackensack, NJ, 2011.

[GKM] M. Goresky, R. Kottwitz, and R. MacPherson, Homology of affine Springer fibres in the unramified case, Duke Math. J. 121 (2004), 509-561.

[HT] M. Harris and R. Taylor, On the Geometry and Cohomology of Some Simple Shimura Varieties, Ann. of Math. Studies 151, Princeton Univ. Press, Princeton, N.J., 2001.

$[\mathrm{H}] \quad$ G. Henniart, Une preuve simple des conjectures de Langlands de $G L(n)$ sur un corps p-adique, Invent. Math. 139 (2000), 439-455.

[JPS] H. Jacquet, I. Piatetski-Shapiro, and J. Shalika, Rankin-Selberg convolutions, Amer. J. Math. 105 (1983), 367-464.

[JS] H. Jacquet and J. Shalika, On Euler products and the classification of automorphic representations II, Amer. J. Math. 103 (1981), 777-815.

[Ji] D. Jiang, Automorphic integral transforms for classical groups I: endoscopy correspondences, to appear in Proceedings on the Conference on Automorphic Forms and Related Geometry: Assessing the Legacy of I.I. Piatetski-Shapiro. 
[K] R. Kottwitz, Stable trace formula: cuspidal tempered terms, Duke Math. J. 51 (1984), 611-650.

[KS] R. Kottwitz and D. Shelstad, Foundations of Twisted Endoscopy, Astérisque, vol. 255.

$[\mathrm{Ku}] \quad \mathrm{S}$. Kudla, Notes on the local theta correspondence, notes from the European School on Group Theory, September 1996.

[L1] R. Langlands, Problems in the theory of automorphic forms, in Lectures in Modern Analysis and Applications, Lecture Notes in Math. 170, Springer, New York, 1970, 18-61.

[L2] - On the Functional Equations Satisfied by Eisenstein Series, Lecture Notes in Math. 544, Springer, New York, 1976.

[L3] - On the notion of an automorphic representation. A supplement to the preceding paper, in Automorphic Forms, Representations and L-functions, Proc. Sympos. Pure Math. vol. 33, Part 1, Amer. Math. Soc., 1979, 203-208.

[L4] - Automorphic representations, Shimura varieties, and motives. Ein Märchen, in Automorphic Forms, Representations and L-functions, Proc. Sympos. Pure Math. vol. 33, Part 2, Amer. Math. Soc., 1979, 205-246.

[L5] - On the classification of irreducible representations of real algebraic groups, in Representation Theory and Harmonic Analysis on Semisimple Lie Groups, AMS Mathematical Surveys and Monographs, vol. 31, 1989, 101-170.

[L6] — Un nouveau point de repère des formes automorphes, Canad. Math. Bull. 50 (2007), 243-267.

[LS] R. Langlands and D. Shelstad, On the definition of transfer factors, Math. Ann. 278 (1987), 219-271.

[Me] P. Mezo, Tempered spectral transfer in the twisted endoscopy of real groups, preprint.

[M1] C. Moeglin, Paquets d'Arthur discrets pour un groupe classique p-adique, Contemp. Math. 489 (2009), 179-257.

[M2] - Multiplicité 1 dans les paquets d'Arthur aux places p-adiques, in On Certain L-functions, Clay Mathematics Proceedings, vol. 13, 2011, 233-274.

[M3] - Conjecture d'Adams pour la correspondence de Howe et filtration de Kudla, in Arithmetic Geometry and Automorphic Forms, 445-503, Adv. Lect. Math. (ALM), 19, Int. Press, Somerville, MA, 2011.

[MW1] C. Moeglin and J.-L. Waldspurger, Le spectre résiduel de $G L(n)$, Ann. Scient. Éc. Norm. Sup. $4^{\mathrm{e}}$ série 22 (1989), 605-674.

[MW2] - La partie géométrique de la formule des traces tardue, preprint.

[Mok] C.P. Mok, Endoscopic classification of representations of quasisplit unitary groups, to appear in Memoires of the American Mathematical Society.

[N] B.C. Ngô, Le lemme fondamental pour les algèbres de Lie, Publ. Math. Inst. Hautes Études Sci. 111, 1-269.

[Sch] P. Scholze, The local Langlands correspondence for $G L_{n}$ over $p$-adic fields, to appear in Invent. Math.

[Sha] F. Shahidi, A proof of Langlands' conjecture on Plancherel measures; Complementary series for p-adic groups, Annals of Math. 132 (1990), 273-330.

[She1] D. Shelstad, L-indistinguishability for real groups, Math. Ann. 259 (1982), 385-430.

[She2] - Tempered endoscopy for real groups I: geometric transfer with canonical factors, Contemp. Math., 472 (2008), 215-246.

[She3] - Tempered endoscopy for real groups II: spectral transfer factors, to appear in Autmorphic Forms and the Langlands Program, Higher Education Press/International Press, 243-283.

[She4] - Tempered endoscopy for real groups III: inversion of transfer and L-packet structure, Representation Theory 12 (2008), 369-402.

[She5] - On geometric transfer in real twisted endoscopy, preprint.

[T] J. Tate, Fourier Analysis in Number Fields and Hecke's Zeta Functions, in Algebraic Number Theory, Thompson, Washington, D.C., 1967, 305-347.

[W1] J.-L. Waldspurger, Le lemme fondamental implique le transfer, Compositio Math. 105 (1997), 153-236.

[W2] - Endoscopie et changement de caractéristique, J. Inst. Math. Jussieu 5 (2006), $423-525$.

[W3] - L'endoscopie tordue n'est pas si tordue, Memoirs of AMS 908 (2008).

[W4] - A propos du lemme fondamental pondéré tordu, Math. Ann. 343 (2009), 103-174. 
[W5] - Préparation à la stabilisation de la formule des traces tordue I: endoscopie tordue sur un corps local, preprint.

[W6] - Préparation à la stabilisation de la formule des traces tordue II: intégrales orbitales et endoscopie sur un corps non-archimédien, preprint

[W7] — Préparation à la stabilisation de la formule des traces tordue III: intégrales orbitales et endoscopie sur un corps archimédien, preprint.

Department of Mathematics, University of Toronto, Toronto, Canada M5S 2E4

E-mail address: arthur@math.toronto.edu 